\title{
Interim Sanitary Landfill Groundwater Monitoring Report (1998 Annual Report)
}

by

D. Wells

Westinghouse Savannah River Company

Savannah River Site

Aiken, South Carolina 29808

This paper was prepared in connection with work done under the above contract number with the U.S. Department of Energy. By acceptance of this paper, the publisher and/or recipient acknowledges the U.S. Government's right to retain a nonexclusive, royalty-free license in and to any copyright covering this paper, along with the right to reproduce and to authorize others to reproduce all or part of the copyrighted paper. 
WSRC-RP-99-00001

Unclassified

\section{INTERIM SANITARY LANDFILL GROUNDWATER MONITORING REPORT (U)}

\section{ANNUAL REPORT \\ January 1999}

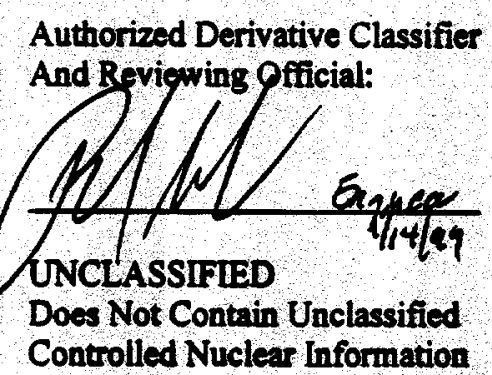

Westinghouse Savannah River Company

Savannah River Sito

Aiken, SC 29808 


\section{DISCLAIMER}

This report was prepared as an account of work sponsored by an agency of the United States Government. Neither the United States Government nor any agency thereof, nor any of their employees, makes any warranty, express or implied, or assumes any legal liability or responsibility for the accuracy, completeness, or usefulness of any information, apparatus, product, or process disclosed, or represents that its use would not infringe privately owned rights. Reference herein to any specific commercial product, process, or service by trade name, trademark, manufacturer, or otherwise does not necessarily constitute or imply its endorsement, recommendation, or favoring by the United States Government or any agency thereof. The views and opinions of authors expressed herein do not necessarily state or reflect those of the United States Government or any agency thereof.

This report has been reproduced directly from the best available copy.

Available to DOE and DOE contractors from the Office of Scientific and Technical Information, P.O. Box 62, Oak Ridge, TN 37831; prices available from (615) 576-8401.

Available to the public from the National Technical Information Service, U.S. Department of Commerce, 5285 Port Royal Road, Springfield, VA 22161. 


\section{DISCLAIMER}

Portions of this document may be illegible in electronic image products. Images are produced from the best available original document. 
WSRC-RP-99-00001

Unclassified

\section{INTERIM SANITARY LANDFILL GROUNDWATER MONITORING REPORT (U)}

\section{ANNUAL REPORT January 1999}

Authorized Derivative Classifier And Reviowing 9 fficial:

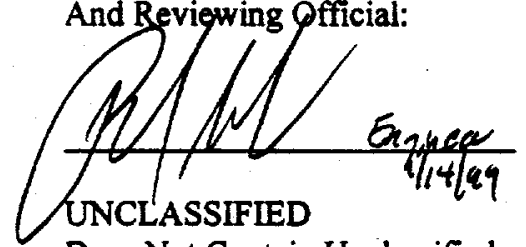

Does Not Contain Unclassified

Controlled Nuclear Information

Westinghouse Savannah River Company

Savannah River Site

Aiken, SC 29808 


\section{INTRODUCTION}

The SRS Interim Sanitary Landfill opened in mid-1992 and operated until 1998 under Domestic Waste Permit \#025500-1120. Several contaminants have been detected in the groundwater beneath the unit. Several metals (chiefly barium) have been detected, but cannot been definitively linked to landfill operations. However, persistent detections of trichlorofluoromethane, 1,1-Dichloroethane, and 1,1,1- trichloroethane are almost certainly indicators of a contaminant release from the unit.

In accordance with the Groundwater Quality Assessment for the Interim Sanitary Landfill, WSRC-RP-97-253, Revision 1 , the 15 wells shown in figure 1 were each sampled twice during 1998. Sampling was done during the first and third quarters of 1998 . The analytical results for all of the wells appear in Appendix I.

The well sampling and analyses were conducted in accordance with Procedure Manual 3Q5, Hydrogeologic Data Collection.

Metals analyses were largely inconclusive, but did indicate possible impacts from the facility. Volatile organic compound analyses indicated that a plume of trichlorofluoromethane continues to grow beneath the landfill. The presence of breakdown products at the center of the plume indicates that some biological degradation is taking place. One new volatile contaminant, acetone, was detected in one well.

Water level data from new wells along the west side of the landfill indicate that there may be a groundwater mound beneath the waste cell. This could not be detected with the pre-1998 well spacing.

\section{DISCUSSION}

\section{Flow Direction and Rate}

The uppermost aquifer beneath the ISL is the Steed Pond Aquifer. All previous studies indicated that flow in the Steed Pond was to the southeast as shown in figure 2. However, data from new wells LFW-76, LFW-77, and LFW-78 indicate that there is also flow to the south and perhaps even to the west (figures 3 and 4 ). This neariy radial flow indicates that groundwater may have mounded below the waste cell.

Since the cell was a low, excavated area it is reasonable to assume that it collected a great deal of runoff. As this accumulated water percolated downward and recharged the aquifer, it could have caused localized mounding of the water table surface.

Flow rate can be estimated using the following equation:

$$
\text { Flow }(\mathrm{ft} / \text { day })=\frac{\text { Hydraulic Conductivity }(\mathrm{ft} / \text { day })}{\text { Porosity (unitless) }} \times \frac{\mathrm{dh}(\mathrm{ft})}{\mathrm{dl}(\mathrm{ft})}
$$

where the hydraulic conductivity constant is $16 \mathrm{ft} /$ day, the effective porosity value is 20 percent, the change in head is $\mathrm{dh}$, and the horizontal distance along each flow arrow is $\mathrm{dl}$.

The flow rate was estimated in two directions indicated by flow arrows $A \cdot A^{\prime}$ and $B-B^{\prime}$ on figures 3 and 4 . For first quarter of 1998 (figure 3), the calculations are as follows: 


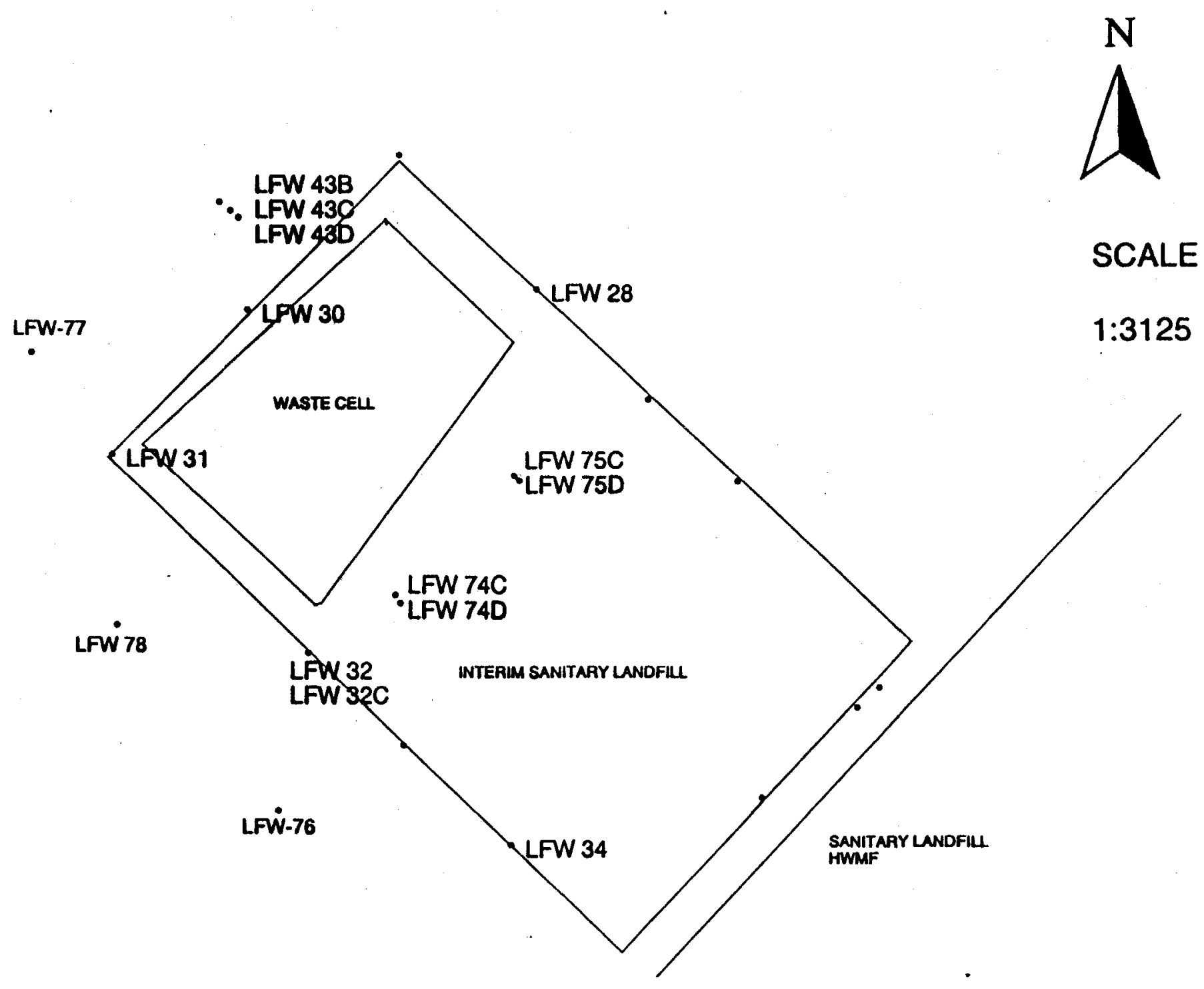

Figure I. Interim Sanitary Landfill monitoring wells. 


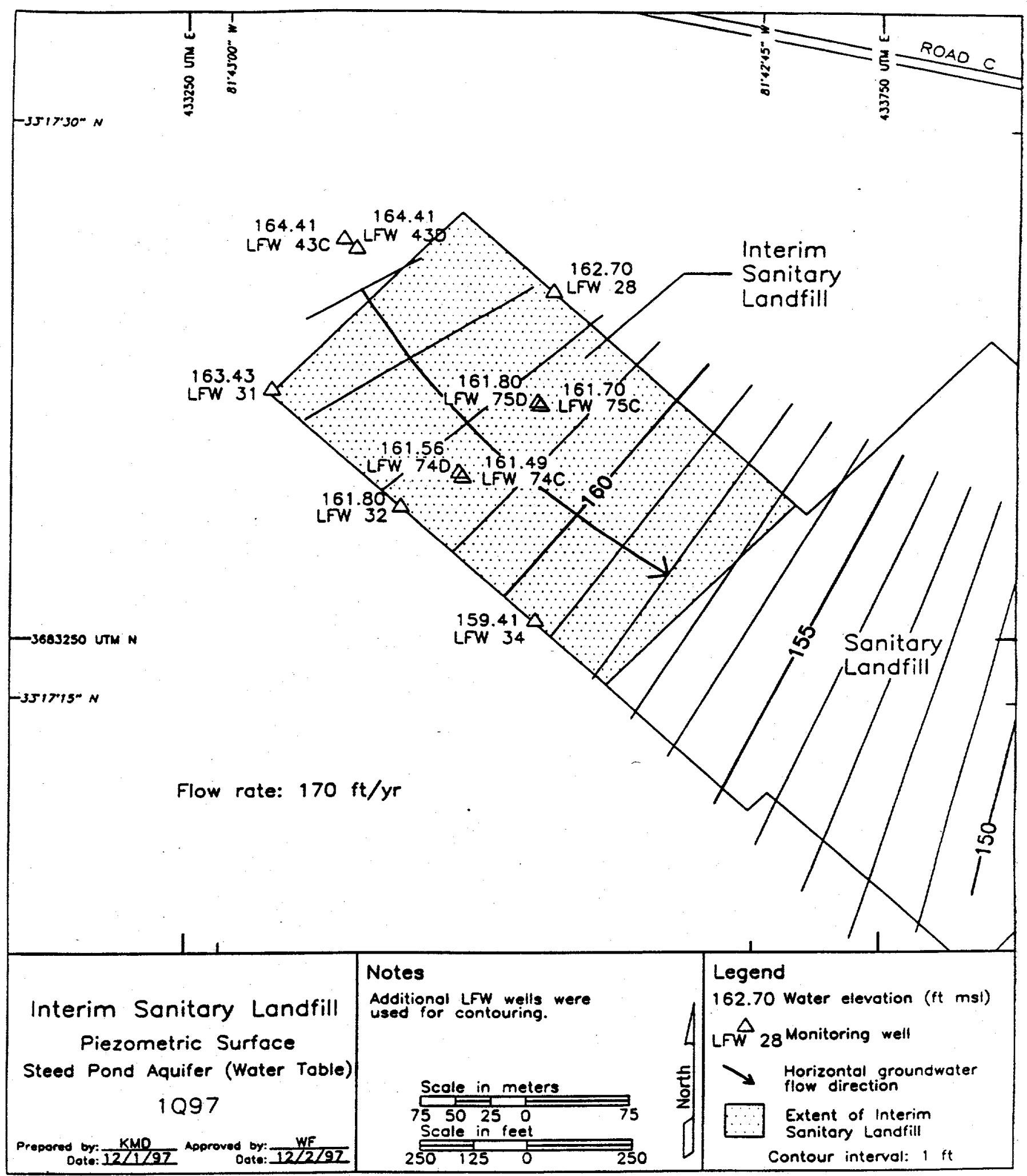

Figure 2. Potentiometric surface map of the Steed Pond Aquifer, first quarter 1997. 

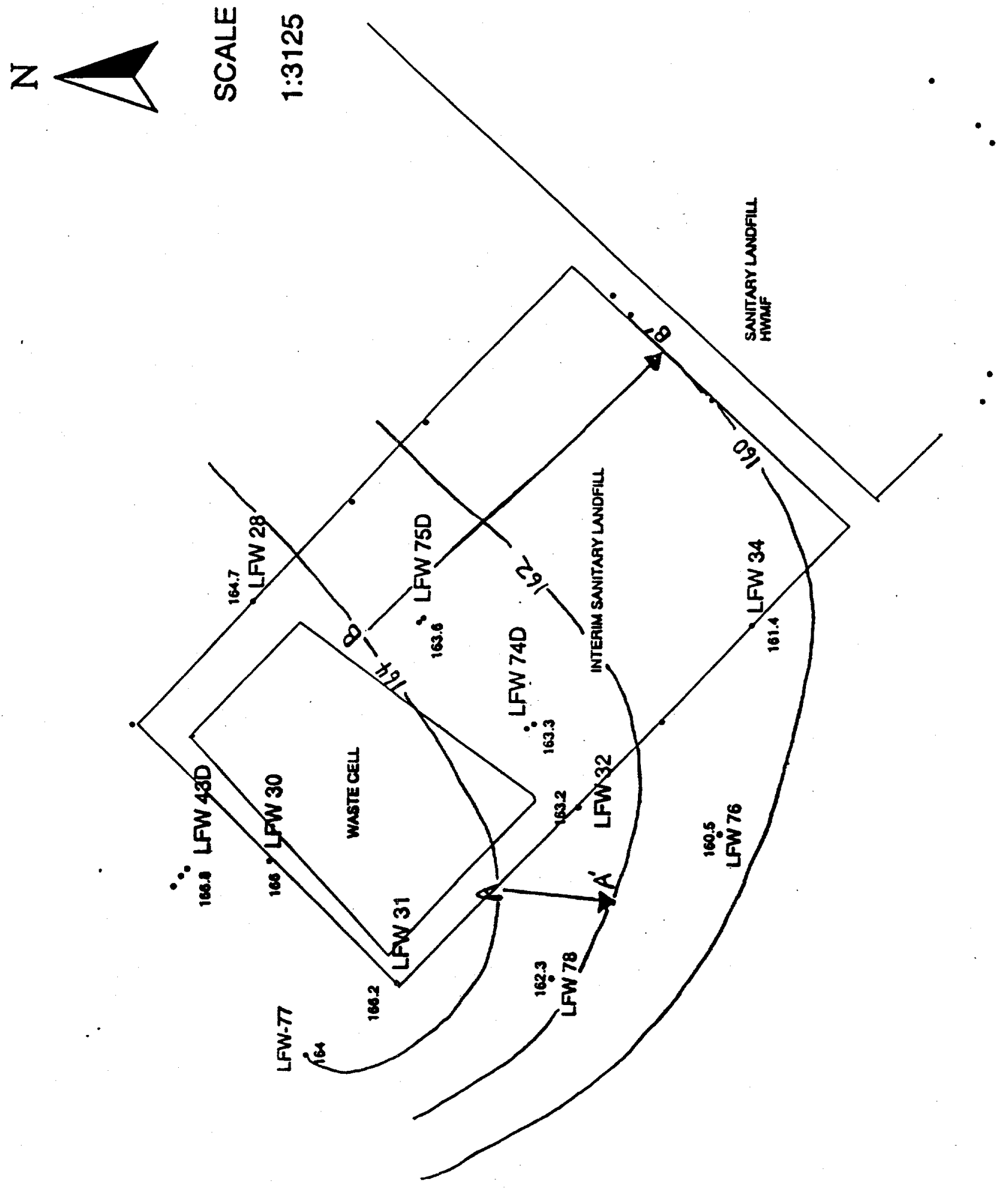


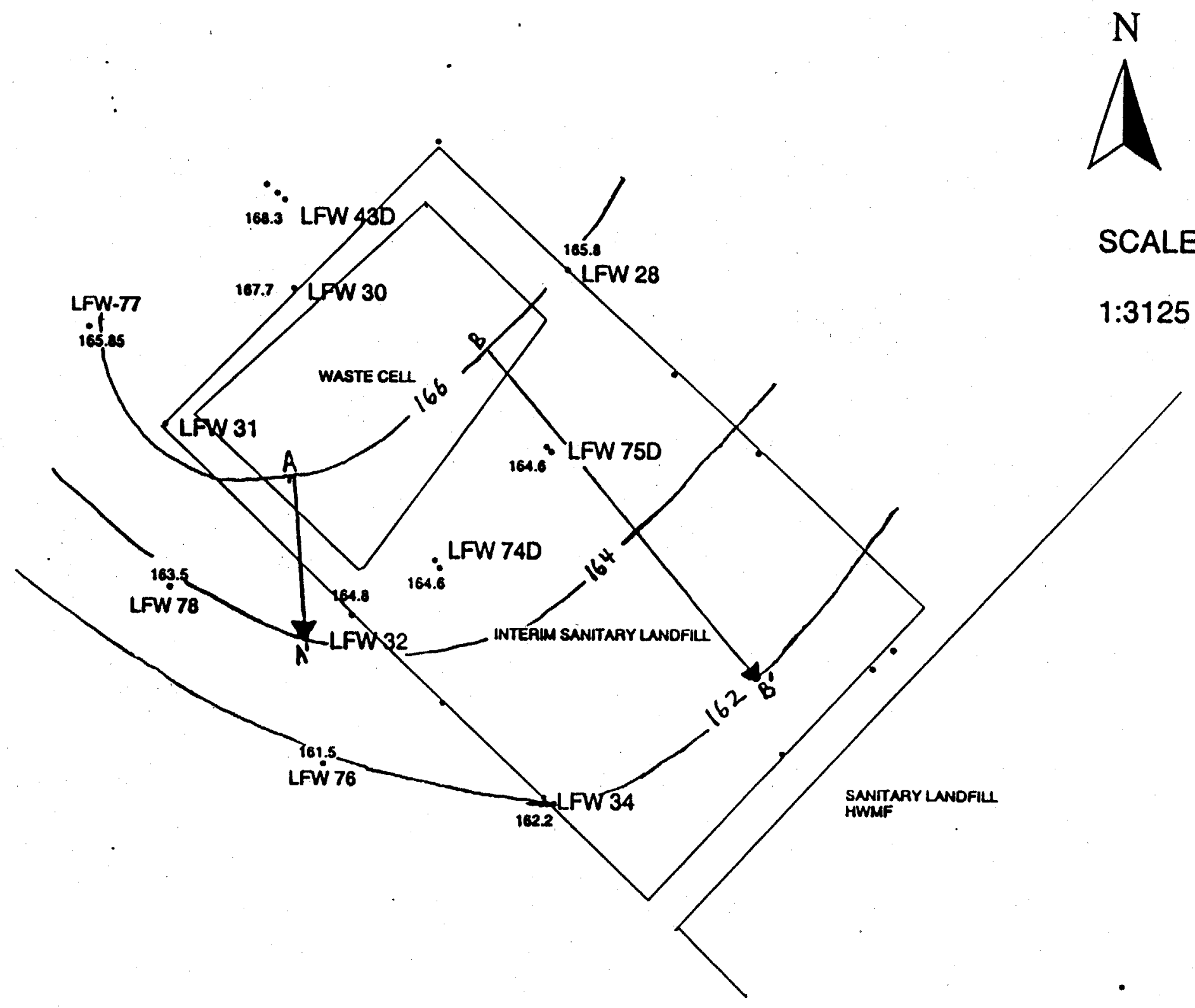

Figure 4. Potentiometric surface map for the Steed Pond Aquifer, third quarter 1998. 
Line $A-A^{\prime}$

$16 / .20 \times 2 \mathrm{ft} / 195.3 \mathrm{ft}=0.8 \mathrm{ft} /$ day or $292 \mathrm{ft} /$ year

Line B-B'

$16 / .20 \times 4 \mathrm{ft} / 716.3 \mathrm{ft}=0.48 \mathrm{ft} /$ day or $175.2 \mathrm{ft} /$ year

For third quarter of 1998 (figure 4), the calculations are as follows:

Line A-A'

$16 / .20 \times 2 \mathrm{ft} / 260.4 \mathrm{ft}=0.64 \mathrm{ft} /$ day or $233.6 \mathrm{ft} /$ year

Line B-B'

$16 / .20 \times 4 \mathrm{ft} / 684.9 \mathrm{ft}=0.48 \mathrm{ft} /$ day or $175.2 \mathrm{ft} /$ year

\title{
Analytical Results
}

The following analytes were detected at levels above background:

\author{
$1,1,1$-trichloroethane \\ 1,1 -dichloroethane \\ 1,2 -dichloroethane \\ trichlorofluoromethane \\ acetone \\ barium \\ chromium \\ cobalt \\ copper \\ lead \\ mercury \\ vanadium \\ zinc
}

\section{1,1,1-trichloroethane, 1,1- dichloroethane, trichlorofluoromethane}

The trichlorofluoromethane plume appeared to spread somewhat between first and third quarters (figures 5 and 6). Concentrations in the most contaminated well, LFW-32, continue to rise. The third quarter concentration in that well was $315 \mathrm{ppb}$.

1,1,1- trichloroethane and 1,1- dichloroethane are probably present as breakdown products of trichlorofluoromethane. Moving downgradient from LFW-32 to LFW-34

trichlorofluoromethane concentrations drop while 1,1,1-trichloroethane concentrations appear to undergo a slight increase. This indicates that the primary contaminant may be undergoing some form of biotransformation.

\section{1-2 dichloroethane}

This contaminant was reported as detected at a level very close to the detection limit ( $7.5 \mathrm{ppb}$ with a detection limit of $5 \mathrm{ppb}$ ) in one sample from the deepest upgradient well (LFW-43B). 


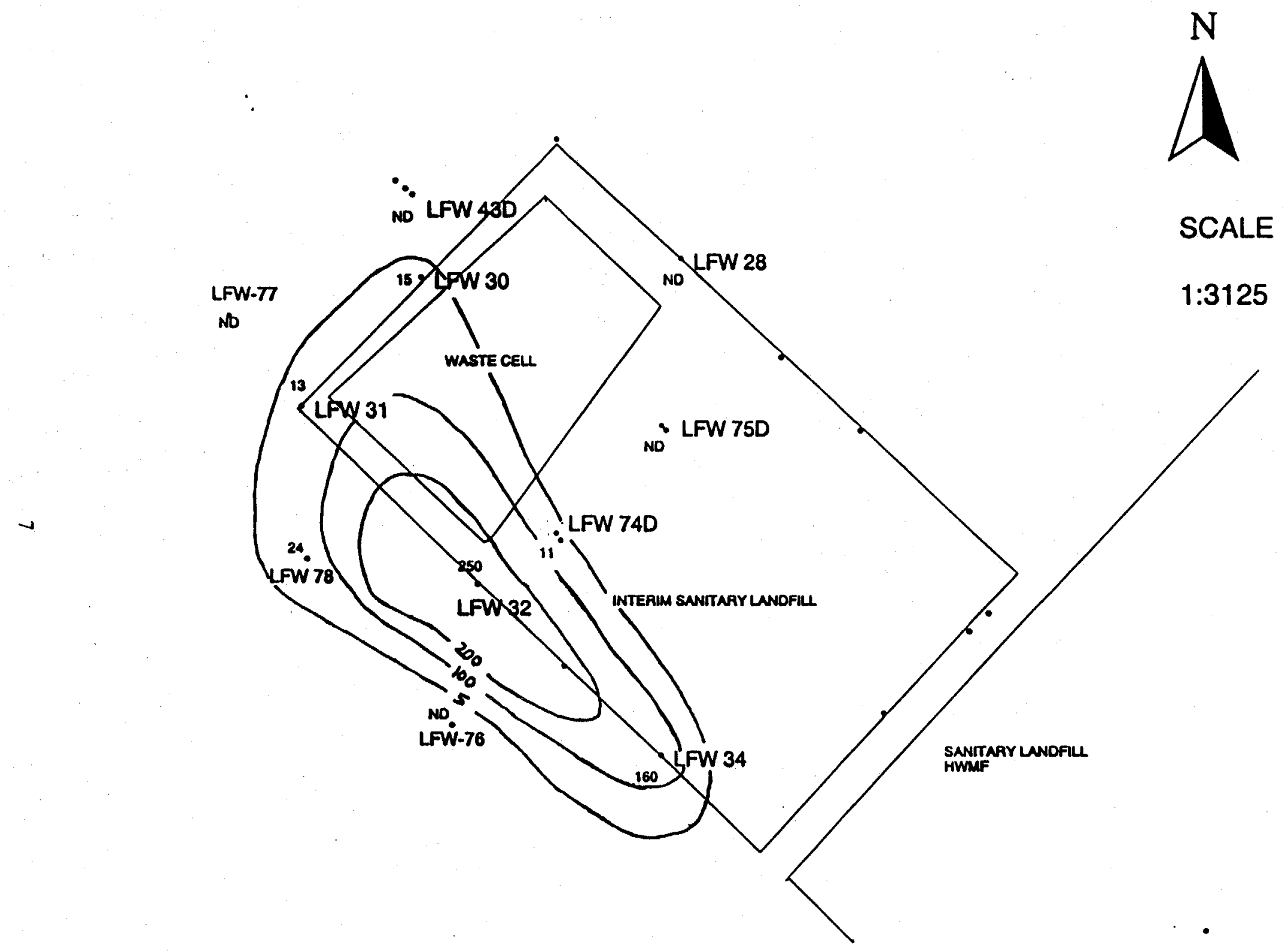

Figure 5. Trichlorofluoromethane isoconcentration map for first quarter 1998 (in ppb). 


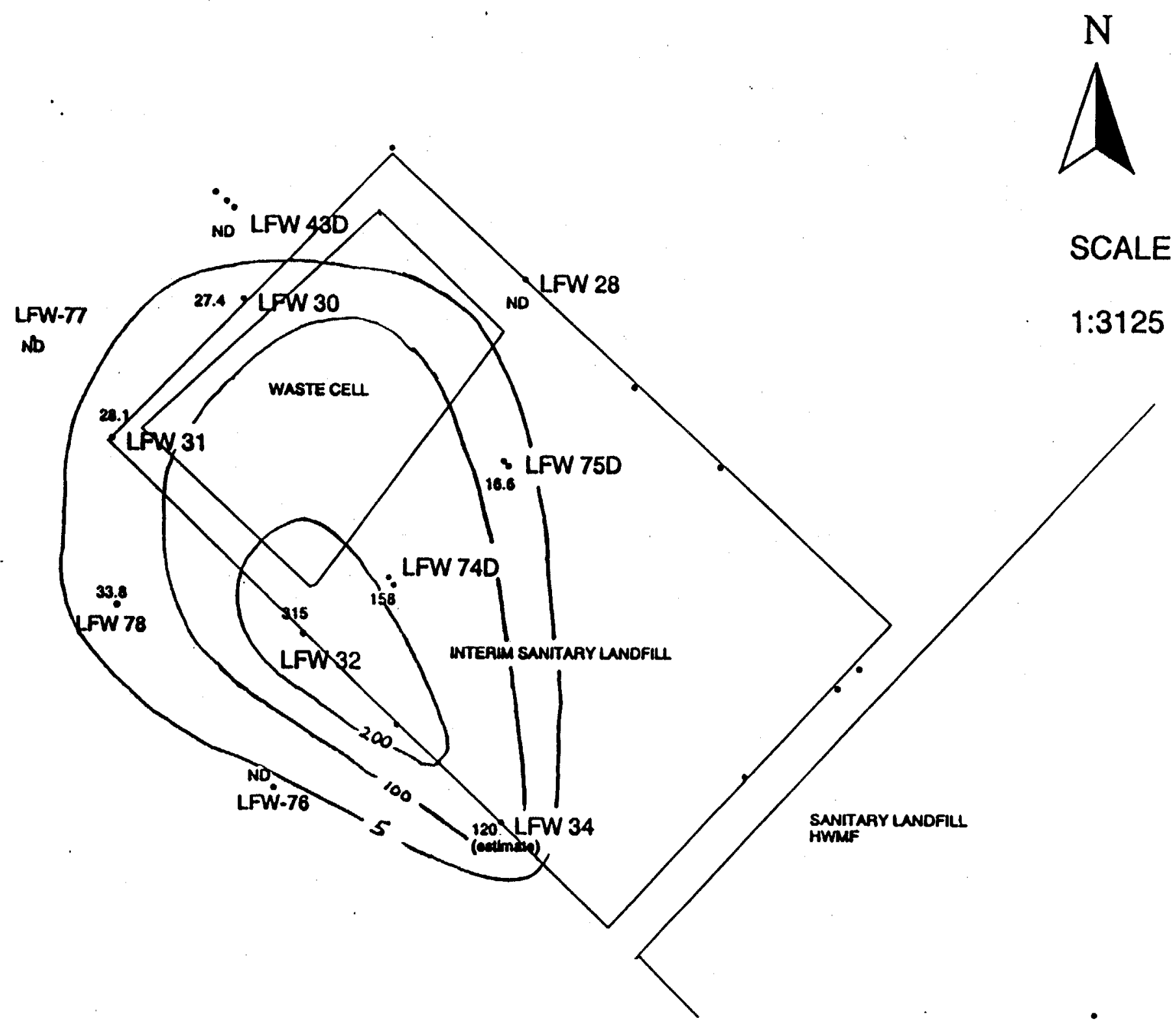

Figure 6. Trichlorofluoromethane isoconcentration map for third quarter 1998 (in ppb). 
At this time the reported result is considered highly questionable. If detections recur, the location of the background cluster will need to be reevaluated.

\section{Acetone}

Acetone was detected in well LFW-74D in both sampling events. Acetone is a common laboratory contaminant, the fact that it showed up in the same well twice and didn't show up in any others indicates that the data are correct.

\section{Barium}

Well LFW-28 consistently yields results above background for barium. During third quarter, the LFW-75D sample was also elevated. These samples may indicate the release of small amounts of barium from the ISL. However the levels, in the $20.30 \mathrm{ppb}$ range, are two orders of magnitude below the health-based limit of $2 \mathrm{ppm}$.

\section{Copper}

Well LFW-31 samples exceeded background for copper (30 ppb). The third quarter sample was the highest at $40.9 \mathrm{ppb}$, but it was far below the $1.3 \mathrm{ppm}$ action level.

\section{Lead}

Background for lead is $18 \mathrm{ppb}$. The first quarter result from LFW-31 was $19.3 \mathrm{ppb}$. Lead was not detected in LFW-31 during third quarter.

\section{Mercury}

Mercury was detected in one sample from LFW-34. The concentration was very low (1.46 ppb), but, in accordance with the monitoring plan, this Appendix II contaminant will be placed on the semiannual monitoring schedule.

\section{Zinc}

Several zinc results were higher than the $29 \mathrm{ppb}$ background level. The highest result was 84 ppb from LFW-75C.

\section{Chromium, Cobalt, Nickel and Vanadium}

Chromium, cobalt, nickel and vanadium were detected only in new monitoring wells with turbid samples. It has been our experience that new wells often yield high metals results. Therefore, these results are not considered significant.

\section{CONCLUSION}

Water level data from the new monitoring wells indicate that groundwater may be mounded beneath the ISL. This would lead to radial or nearly radial flow in a small area around the waste cell, although the overall regional flow would still be to the southeast. This condition, if it really exists, may disappear now that the waste cell has been filled and covered.

Several inorganics were detected in the monitoring wells. As a result of its detection, mercury will henceforth be monitored semiannually along with the Appendix I analytes, but none of the other inorganic detections warrant any action at this time. 
The continuing increase in trichlorofluoromethane concentrations is of concern. But the presence of possible breakdown products indicates biotransformation may be occurring. Acetone, a newly detetected organic, will be monitored with great interest. 
APPENDIX I

ANALYTICAL DATA 



\begin{tabular}{|c|}
\hline Well Nam \\
\hline PFW 28 \\
\hline LFW 28 \\
\hline LFW 28 \\
\hline LW 28 \\
\hline LFW 2G \\
\hline LW 28 \\
\hline LFW 28 \\
\hline LFW 28 \\
\hline LFW 28 \\
\hline LFW 28 \\
\hline LFW 28 \\
\hline LFW 23 \\
\hline LFW 28 \\
\hline LFW 28 \\
\hline LFW 28 \\
\hline LFW 28 \\
\hline LFW 20 \\
\hline LFW 28 \\
\hline LFW 2: \\
\hline LFW 28 \\
\hline LFW 28 \\
\hline LFW 26 \\
\hline LFW 28 \\
\hline FW 20 \\
\hline LFW 20 \\
\hline LFW \\
\hline LFW 20 \\
\hline LFW 28 \\
\hline LFW 28 \\
\hline LFW 28 \\
\hline LFW 20 \\
\hline LFW 28 \\
\hline LFW 28 \\
\hline LFW 26 \\
\hline LFW 20 \\
\hline LFW 28 \\
\hline LFW 20 \\
\hline LFW 28 \\
\hline LFW 26 \\
\hline IF 28 \\
\hline LW 28 \\
\hline LFW 28 \\
\hline LF 20 \\
\hline LFW 26 \\
\hline LFW 28 \\
\hline LF 20 \\
\hline एक 20 \\
\hline LFW 28 \\
\hline IF 2 \\
\hline LW 28 \\
\hline LFW 20 \\
\hline LF 23 \\
\hline LFW 29 \\
\hline LFW 20 \\
\hline LFW 28 \\
\hline LFW 26 \\
\hline LFW 26 \\
\hline LFW 20 \\
\hline LFW 20 \\
\hline LFW 20 \\
\hline LFW 20 \\
\hline LFW 20 \\
\hline LFW 20 \\
\hline LFW 20 \\
\hline LFW 20 \\
\hline LW 28 \\
\hline ज्W 20 \\
\hline LW20 \\
\hline LFW 20 \\
\hline LW2 \\
\hline LFW 28 \\
\hline LFW 26 \\
\hline LFW 28 \\
\hline LFW 26 \\
\hline LFW 28 \\
\hline LF 28 \\
\hline LFW 30 \\
\hline LFW 30 \\
\hline LFW 30 \\
\hline LFW 30 \\
\hline LW 30 \\
\hline LFW 30 \\
\hline$L^{3}{ }^{30}$ \\
\hline LFW 30 \\
\hline LFW 30 \\
\hline LFW 30 \\
\hline LFW 30 \\
\hline LFW 30 \\
\hline LFW 30 \\
\hline LFW 30 \\
\hline LFW \\
\hline LF 30 \\
\hline LFW 30 \\
\hline LW 30 \\
\hline LFW 30 \\
\hline LFW 30 \\
\hline LFW 30 \\
\hline
\end{tabular}




\begin{tabular}{|c|}
\hline Wet Name \\
\hline LFW 30 \\
\hline LFW 30 \\
\hline LFW 30 \\
\hline LFW 30 \\
\hline LFW 30 \\
\hline LFW 30 \\
\hline LFW 30 \\
\hline LFW 30 \\
\hline LFW 30 \\
\hline LNW 30 \\
\hline LFW 30 \\
\hline LFW 30 \\
\hline LFW 30 \\
\hline LFW 30 \\
\hline LFW 30 \\
\hline LFW 30 \\
\hline LFW 30 \\
\hline LFW 30 \\
\hline LFW 30 \\
\hline LFW 30 \\
\hline LFW 30 \\
\hline LW 30 \\
\hline LFW 30 \\
\hline LFW 30 \\
\hline LFW 30 \\
\hline LFW 30 \\
\hline LFW 30 \\
\hline LFW 30 \\
\hline LFW 30 \\
\hline LW 30 \\
\hline LFW 30 \\
\hline LFW30 \\
\hline LFW 30 \\
\hline 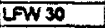 \\
\hline LFW 30 \\
\hline LFW 30 \\
\hline LFW 30 \\
\hline LFW 30 \\
\hline FW 30 \\
\hline LFW 30 \\
\hline LFW 30 \\
\hline LW \\
\hline LFW 30 \\
\hline LW 30 \\
\hline LFW30 \\
\hline LFW 30 \\
\hline LFW 30 \\
\hline$\angle F W 30$ \\
\hline LFW 30 \\
\hline LFW 30 \\
\hline LFW 30 \\
\hline LFW 30 \\
\hline FW 30 \\
\hline LFW 30 \\
\hline LFW 30 \\
\hline LFW 31 \\
\hline FW 31 \\
\hline LFW 31 \\
\hline LFW 31 \\
\hline LFW 31 \\
\hline LFW 31 \\
\hline LFW 31 \\
\hline LFW 31 \\
\hline LFW 31 \\
\hline LFW 31 \\
\hline LFW 31 \\
\hline LFW 31 \\
\hline LW34 \\
\hline LFW 31 \\
\hline LFW 31 \\
\hline LFW \\
\hline LFW 31 \\
\hline LFW3 \\
\hline LFW 31 \\
\hline LFW 31 \\
\hline LFW 31 \\
\hline LFW 31 \\
\hline LFW 31 \\
\hline Lพ \\
\hline LFW \\
\hline LF 31 \\
\hline LFW 31 \\
\hline LFW 31 \\
\hline LW 31 \\
\hline LF 31 \\
\hline LFW 31 \\
\hline LW 31 \\
\hline LFW31 \\
\hline LFW 31 \\
\hline LF 31 \\
\hline LFW 31 \\
\hline LFW31 \\
\hline LFW 34 \\
\hline LF 31 \\
\hline LFW 31 \\
\hline LFW 31 \\
\hline LFW31 \\
\hline
\end{tabular}




\begin{tabular}{|c|}
\hline Wefl Nam \\
\hline LFW 34 \\
\hline LFW 31 \\
\hline FW 31 \\
\hline$\angle F W 39$ \\
\hline LFW 31 \\
\hline LFW 31 \\
\hline LFW31 \\
\hline LFW 39 \\
\hline LFW31 \\
\hline LFW 31 \\
\hline LFW 31 \\
\hline LFW 31 \\
\hline LFW 31 \\
\hline LFW 34 \\
\hline LFW31 \\
\hline$L F W / 31$ \\
\hline UF 31 \\
\hline LF 31 \\
\hline LFW 31 \\
\hline LFW 31 \\
\hline LFW 31 \\
\hline LFW31 \\
\hline LFW 31 \\
\hline LFW 31 \\
\hline FF 31 \\
\hline LFW31 \\
\hline LFW31 \\
\hline LFW3 \\
\hline LFW 31 \\
\hline LFW 39 \\
\hline LW31 \\
\hline LFW 31 \\
\hline LFW 31 \\
\hline L्W \\
\hline LFW 31 \\
\hline LFW \\
\hline LFW 31 \\
\hline LFW 31 \\
\hline LFW31 \\
\hline LF 31 \\
\hline LFW31 \\
\hline LFW31 \\
\hline LFW 31 \\
\hline LFW31 \\
\hline LFW 31 \\
\hline LFW31 \\
\hline LFW 31 \\
\hline FW31 \\
\hline LFW 31 \\
\hline LFW 31 \\
\hline LFW31 \\
\hline LFW 31 \\
\hline LFW 39 \\
\hline LFW31 \\
\hline LFW 31 \\
\hline LFW34 \\
\hline LFW 31 \\
\hline LFW 31 \\
\hline LFW31 \\
\hline LFW31 \\
\hline LW 31 \\
\hline LW 31 \\
\hline LW31 \\
\hline LFW 31 \\
\hline LFW 31 \\
\hline LFW31 \\
\hline LFW 31 \\
\hline LFW31 \\
\hline LFW 31 \\
\hline LFW 31 \\
\hline L्W31 \\
\hline LFW 31 \\
\hline LFW 31 \\
\hline LFW \\
\hline LW \\
\hline FW 31 \\
\hline LFW31 \\
\hline LF 31 \\
\hline LFW 31 \\
\hline LWISt \\
\hline LFW $3 t$ \\
\hline fow 31 \\
\hline LFW 31 \\
\hline LFW 31 \\
\hline FW31 \\
\hline LFW31 \\
\hline LFW31 \\
\hline LFW 31 \\
\hline LFW 31 \\
\hline LFW 31 \\
\hline LFW31 \\
\hline LFW 34 \\
\hline LFW \\
\hline LFW \\
\hline LFW31 \\
\hline LFW 31 \\
\hline fort31 \\
\hline
\end{tabular}




\begin{tabular}{|c|}
\hline Woll Ham \\
\hline LFW34 \\
\hline LFW31 \\
\hline LFW 31 \\
\hline LFW31 \\
\hline LFW 39 \\
\hline LFW 31 \\
\hline LFW31 \\
\hline LFW 31 \\
\hline LFW 31 \\
\hline LFW 31 \\
\hline LFW 31 \\
\hline LFW 31 \\
\hline LFW 31 \\
\hline LFW 31 \\
\hline LFW 31 \\
\hline LFW 31 \\
\hline LFW 31 \\
\hline LFW 31 \\
\hline LFW \\
\hline LFW 31 \\
\hline LFW 31 \\
\hline LFW31 \\
\hline LFW 31 \\
\hline LFW31 \\
\hline LFW 31 \\
\hline LFW 31 \\
\hline LW 31 \\
\hline LFW 31 \\
\hline LFW31 \\
\hline FW 31 \\
\hline LFW31 \\
\hline LFW 31 \\
\hline LFW 31 \\
\hline FW 31 \\
\hline LFW 31 \\
\hline LFW 31 \\
\hline LFW 31 \\
\hline LFW 31 \\
\hline FW31 \\
\hline LFW 31 \\
\hline LFW 31 \\
\hline LFW31 \\
\hline FW31 \\
\hline TF 31 \\
\hline IFW 31 \\
\hline LF 31 \\
\hline LFW 31 \\
\hline FFW \\
\hline LFW 31 \\
\hline LF 31 \\
\hline LF 31 \\
\hline LFW 31 \\
\hline IN 31 \\
\hline EW 31 \\
\hline LFW 32 \\
\hline LFW32 \\
\hline LFW 32 \\
\hline LFW 32 \\
\hline LFW 32 \\
\hline LFW 32 \\
\hline LFW 32 \\
\hline LF 32 \\
\hline LFW 32 \\
\hline LFW 32 \\
\hline WW 32 \\
\hline LFW 32 \\
\hline LFW 32 \\
\hline LFW 32 \\
\hline LFW 32 \\
\hline LFW 32 \\
\hline LFW 32 \\
\hline LFW 32 \\
\hline LFW 32 \\
\hline LFW 32 \\
\hline LFW 32 \\
\hline LFW 32 \\
\hline LFW 32 \\
\hline $5 \times 32$ \\
\hline LF 32 \\
\hline LF 32 \\
\hline LW 32 \\
\hline 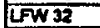 \\
\hline LF 32 \\
\hline LFW 32 \\
\hline LFW32 \\
\hline LFW 32 \\
\hline LFW 32 \\
\hline LFW 32 \\
\hline LFW 32 \\
\hline LFW 32 \\
\hline FW 12 \\
\hline LFW 32 \\
\hline LFW 32 \\
\hline LFW 32 \\
\hline LFW 32 \\
\hline LFW32 \\
\hline LFW 32 \\
\hline
\end{tabular}




\begin{tabular}{|c|}
\hline Woll Name \\
\hline LF 32 \\
\hline LFW 32 \\
\hline LFW 12 \\
\hline LFW 32 \\
\hline DFW 32 \\
\hline LFW 32 \\
\hline LFW 32 \\
\hline LFW 32 \\
\hline LF 32 \\
\hline IF 32 \\
\hline LFW 32 \\
\hline LFW 32 \\
\hline LFW 32 \\
\hline LFW 32 \\
\hline LFW 32 \\
\hline LFW 32 \\
\hline LFW 32 \\
\hline LFW 32 \\
\hline FW 32 \\
\hline LW 32 \\
\hline LFW 32 \\
\hline LFW 32 \\
\hline LFW 32 \\
\hline LFW 32 \\
\hline LW 32 \\
\hline LFW 32 \\
\hline LFW 32 \\
\hline LFW 32 \\
\hline LFW 32 \\
\hline LFW 32 \\
\hline LFW 32 \\
\hline LFW 32 \\
\hline LFW 32 \\
\hline$L F W 32$ \\
\hline WW 32 \\
\hline LFW 32 \\
\hline LFW 32 \\
\hline LFW 32 \\
\hline LW 32 \\
\hline LW 32 \\
\hline LFW 32 \\
\hline LFW 32 \\
\hline LFW32 \\
\hline LFW 32 \\
\hline LFW 32 \\
\hline LFW 32 \\
\hline LFW 32 \\
\hline LFW 32 \\
\hline LFW 32 \\
\hline LFW 32 \\
\hline LFW 32 \\
\hline LFW 32 \\
\hline LFW 32 \\
\hline LFW 32 \\
\hline LFW 32 \\
\hline Low 32 \\
\hline LFW 32 \\
\hline $5 \times 132$ \\
\hline LFW 32 \\
\hline LFW 32 \\
\hline LFW 32 \\
\hline LFW 32 \\
\hline LFW 32 \\
\hline $1 F W 32$ \\
\hline FW 32 \\
\hline LFW 32 \\
\hline LFW 32 \\
\hline LFW 32 \\
\hline LFW 32 \\
\hline FW 32 \\
\hline LF 32 \\
\hline$\angle F W 32$ \\
\hline LF 32 \\
\hline LFW 32 \\
\hline LFW 32 \\
\hline LFW 32 \\
\hline LF 32 \\
\hline LFW 32 \\
\hline LFW 32 \\
\hline LFW 32 \\
\hline FW 32 \\
\hline LFW 32 \\
\hline $1 F W 32$ \\
\hline LFW 32 \\
\hline LFW \\
\hline LFW 32 \\
\hline IF 32 \\
\hline LFW 32 \\
\hline LFW 32 \\
\hline IFW 32 \\
\hline LFW32 \\
\hline LFW 32 \\
\hline - 5132 \\
\hline LFW 32 \\
\hline LF 32 \\
\hline LFW 32 \\
\hline LFW/32 \\
\hline
\end{tabular}




\begin{tabular}{|c|}
\hline Woll Name \\
\hline LFW 32 \\
\hline FW 32 \\
\hline LFW 32 \\
\hline LW 32 \\
\hline LFW 32 \\
\hline LFW 32 \\
\hline LFW 32 \\
\hline FF 32 \\
\hline LFW 32 \\
\hline FW 32 \\
\hline LF 32 \\
\hline LFW 32 \\
\hline LW 32 \\
\hline LFW 32 \\
\hline LFW 32 \\
\hline FW 32 \\
\hline LFW32 \\
\hline LFW 32 \\
\hline LFW 32 \\
\hline LFW 32 \\
\hline LFW 32 \\
\hline LFW 32 \\
\hline LFW 32 \\
\hline LFW \\
\hline LFW 32 \\
\hline LFW 32 \\
\hline LFW 32 \\
\hline$L=W 32$ \\
\hline LFW 32 \\
\hline LW 32 \\
\hline LWW32 \\
\hline LFW 32 \\
\hline LFW 32 \\
\hline LFW 32 \\
\hline LFW 32 \\
\hline LFW 32 \\
\hline FW 32 \\
\hline LFW 32 \\
\hline LFW32 \\
\hline LFW 32 \\
\hline SFW 12 \\
\hline LFW 32 \\
\hline LFW32 \\
\hline LF 32 \\
\hline LW32 \\
\hline LFW 32 \\
\hline LFW 32 \\
\hline LFW 32 \\
\hline Low \\
\hline LW 32 \\
\hline LW 32 \\
\hline LW 32 \\
\hline LW 32 \\
\hline LW 32 \\
\hline LFW 32 \\
\hline LFW 32 \\
\hline LFW 32 \\
\hline LFW 32 \\
\hline LFW 32 \\
\hline LFW 32 \\
\hline LFW 32 \\
\hline FW 32 \\
\hline LFW 32 \\
\hline LFW 32 \\
\hline LFW $32 C$ \\
\hline LFW $32 \mathrm{C}$ \\
\hline LFW 32C \\
\hline LW $32 C$ \\
\hline LFW 32C \\
\hline LFW 32C \\
\hline LF 32C \\
\hline LFW 320 \\
\hline LFW 32C \\
\hline FW 32C \\
\hline LFW 32C \\
\hline LF 32C \\
\hline LFW 320 \\
\hline LFW 320 \\
\hline LFW 32 \\
\hline SW $32 \mathrm{C}$ \\
\hline LFW 320 \\
\hline$L F W$ \\
\hline LW 320 \\
\hline LFW 32C \\
\hline LFW $32 C$ \\
\hline LFW 320 \\
\hline LFW 32C \\
\hline LFW $32 \mathrm{C}$ \\
\hline LFW $32 C$ \\
\hline LFW 326 \\
\hline LFW $32 \mathrm{C}$ \\
\hline LFW 32C \\
\hline $5 \times 320$ \\
\hline LFW 32C \\
\hline LFW 32C \\
\hline LFW 32C \\
\hline LFW 32C \\
\hline
\end{tabular}




\begin{tabular}{|c|}
\hline Well Neme \\
\hline LFW 32C \\
\hline LW 32C \\
\hline LFW $32 C$ \\
\hline LFW $32 \mathrm{C}$ \\
\hline LFW 32C \\
\hline LFW $32 C$ \\
\hline LFW $32 \mathrm{C}$ \\
\hline LW $32 C$ \\
\hline LFw $32 x$ \\
\hline LFW 32C \\
\hline LFW $32 \mathrm{C}$ \\
\hline LFW $32 \mathrm{C}$ \\
\hline LFW $32 C$ \\
\hline LFW $32 \mathrm{C}$ \\
\hline LFW $32 \mathrm{C}$ \\
\hline LFW $32 \mathrm{C}$ \\
\hline LFW $32 \mathrm{C}$ \\
\hline LFW 32C \\
\hline LFW 320 \\
\hline fFW 32C \\
\hline LFW 32C \\
\hline LFW $32 \mathrm{C}$ \\
\hline LFW 32C \\
\hline LFW $32 C$ \\
\hline LFW $32 C$ \\
\hline LFW $32 \mathrm{C}$ \\
\hline LF $32 C$ \\
\hline LFW $32 C$ \\
\hline LF 32C \\
\hline LFW $3 x$ \\
\hline LFW 320 \\
\hline LFW 32C \\
\hline LFW $3 x$ \\
\hline LFW 32C \\
\hline LFW $32 C$ \\
\hline LFW $33 C$ \\
\hline LFW $3 X \mathrm{X}$ \\
\hline LFW $32 \mathrm{C}$ \\
\hline$L F=32 C$ \\
\hline LFW 32C \\
\hline LFW 320 \\
\hline LFW $32 C$ \\
\hline LFW 32C \\
\hline LFW 32C \\
\hline LFW $32 C$ \\
\hline LFW $32 C$ \\
\hline LFW $32 C$ \\
\hline LFW 326 \\
\hline LFW 32C \\
\hline LFW 32C \\
\hline LFW $32 \mathrm{C}$ \\
\hline LF $32 C$ \\
\hline FW $32 c$ \\
\hline LF $32 C$ \\
\hline IFW 32C \\
\hline FW 32C \\
\hline LFW 32C \\
\hline $5 \mathrm{~W} 32 \mathrm{C}$ \\
\hline LFW 326 \\
\hline LFW $32 \mathrm{C}$ \\
\hline LW $32 x$ \\
\hline LFW $3 x$ \\
\hline LF $32 \mathrm{C}$ \\
\hline LFW $32 \mathrm{C}$ \\
\hline LFW $32 C$ \\
\hline LFW $32 c$ \\
\hline LFW 32C \\
\hline LFW $32 \mathrm{C}$ \\
\hline LFW $32 C$ \\
\hline LFW.32C \\
\hline LFW 32C \\
\hline LFW 32C \\
\hline LFW 32C \\
\hline LW $32 C$ \\
\hline LFW 3ZC \\
\hline FW $32 \mathrm{C}$ \\
\hline LFW $32 C$ \\
\hline LFW 32C \\
\hline LFW $32 C$ \\
\hline LFW $32 C$ \\
\hline LFW $32 C$ \\
\hline LFW 32C \\
\hline LFW $32 \mathrm{C}$ \\
\hline LFW 32C \\
\hline LFW 32C \\
\hline LFW 32C \\
\hline LFW 32C \\
\hline sw $32 c$ \\
\hline LFW $32 x$ \\
\hline LFW $32 C$ \\
\hline LFW $3 X$ \\
\hline LFW $32 C$ \\
\hline FW $32 C$ \\
\hline LFW $32 C$ \\
\hline LFW 3xC \\
\hline LW 320 \\
\hline LW 326 \\
\hline
\end{tabular}




\begin{tabular}{|c|}
\hline Well Momo \\
\hline LFW 32C \\
\hline LFW 32C \\
\hline LFW 32C \\
\hline LFW 32C \\
\hline LFW 320 \\
\hline LFW 32C \\
\hline LFW 32C \\
\hline LFW $32 C$ \\
\hline LFW 32C \\
\hline LFW 326 \\
\hline LFW 32C \\
\hline$\angle F W 32 C$ \\
\hline$L F W 32 C$ \\
\hline LFW $32 x$ \\
\hline LFW 32C \\
\hline LFW $32 C$ \\
\hline LFW \\
\hline LFW 32C \\
\hline LFW 32C \\
\hline LFW 32C \\
\hline LFW 32C \\
\hline LFW $32 C$ \\
\hline LFW 32C \\
\hline LFW 32C \\
\hline FW 32C \\
\hline FFW 32C \\
\hline LFW $32 C$ \\
\hline LF $32 C$ \\
\hline LFW 32C \\
\hline LFW $32 C$ \\
\hline LFW32C \\
\hline FW $32 \mathrm{C}$ \\
\hline LFW 32C \\
\hline LFW 32C \\
\hline LF 32C \\
\hline LFW 32C \\
\hline LFW 32C \\
\hline FW 32C \\
\hline LFW 32C \\
\hline LF 32C \\
\hline FW 32C \\
\hline FW 32C \\
\hline LW 32C \\
\hline LF $32 \mathrm{C}$ \\
\hline FW 32C \\
\hline LFW 32C \\
\hline LFW.32C \\
\hline FW $33 C$ \\
\hline LFW 32C \\
\hline LFW $32 C$ \\
\hline LF 32C \\
\hline LFW 32C \\
\hline LF $32 \bar{C}$ \\
\hline LFW $32 \mathrm{C}$ \\
\hline LFW 32C \\
\hline LFW 32C \\
\hline LFW $32 \mathrm{C}$ \\
\hline LFW 32C \\
\hline LFW 32C \\
\hline LFW 32C \\
\hline LFW J2C \\
\hline LFW $32 C$ \\
\hline LFW $32 C$ \\
\hline LW 32C \\
\hline LFW 32C \\
\hline LF $32 \mathrm{C}$ \\
\hline LFW 32C \\
\hline LFW 32C \\
\hline LW $32 C$ \\
\hline LFW $32 \mathrm{C}$ \\
\hline L्W $32 \mathrm{C}$ \\
\hline LW $32 \mathrm{C}$ \\
\hline LFW $32 C$ \\
\hline LFW $32 \mathrm{C}$ \\
\hline LFW 34 \\
\hline NW3 \\
\hline LFW 34 \\
\hline LFW 34 \\
\hline LW 34 \\
\hline Lw34 \\
\hline LFW34 \\
\hline LFW 34 \\
\hline IFW \\
\hline LFW \\
\hline LFW 34 \\
\hline LFW 34 \\
\hline LFW34 \\
\hline FW34 \\
\hline LF 34 \\
\hline LF 34 \\
\hline LW 34 \\
\hline FW 3 \\
\hline LFW 34 \\
\hline LFW 34 \\
\hline IFW 34 \\
\hline LFW 34 \\
\hline Fis \\
\hline
\end{tabular}




\begin{tabular}{|c|}
\hline Well Namo \\
\hline LFW 34 \\
\hline LFW 34 \\
\hline LFW 34 \\
\hline LFW 34 \\
\hline LFW 34 \\
\hline LFW \\
\hline LFW 34 \\
\hline LFW 34 \\
\hline LFW 34 \\
\hline LFW 34 \\
\hline LFW 34 \\
\hline LW 34 \\
\hline LFW 34 \\
\hline LFW 34 \\
\hline LFW 34 \\
\hline LFW 34 \\
\hline LW 34 \\
\hline एक 34 \\
\hline LFW 34 \\
\hline LFW 34 \\
\hline$L F W 34$ \\
\hline LFW 34 \\
\hline LFW 34 \\
\hline LFW 34 \\
\hline LFW 34 \\
\hline LFW 3i \\
\hline LF 34 \\
\hline FW 34 \\
\hline LFW 34 \\
\hline LFW 34 \\
\hline LFW 34 \\
\hline LFW 34 \\
\hline LFW 34 \\
\hline जW \\
\hline LFW 34 \\
\hline LFW34 \\
\hline LFW \\
\hline LFW 36 \\
\hline LFW 34 \\
\hline LFW 3 \\
\hline LFW 3A \\
\hline LFW \\
\hline LFW 34 \\
\hline LFW 34 \\
\hline FW 34 \\
\hline LFW 34 \\
\hline LFW 34 \\
\hline LFW 34 \\
\hline LFW 34 \\
\hline LFW 34 \\
\hline LFW 34 \\
\hline LF 34 \\
\hline LFW 34 \\
\hline LFW 34 \\
\hline LW 34 \\
\hline LFW 34 \\
\hline LFw 34 \\
\hline LFW 34 \\
\hline LFW 34 \\
\hline LFW 34 \\
\hline LFW34 \\
\hline LFW 34 \\
\hline LFW 34 \\
\hline LFW 34 \\
\hline LFW 34 \\
\hline LFW 34 \\
\hline LFW 34 \\
\hline LFW 34 \\
\hline LFW 34 \\
\hline LFW 34 \\
\hline LFW 34 \\
\hline LFW 34 \\
\hline $25 W^{3}$ \\
\hline LFW 34 \\
\hline LFW 34 \\
\hline LFW 3 \\
\hline LFW 34 \\
\hline LFW 34 \\
\hline LFW 34 \\
\hline LFW 34 \\
\hline LW 34 \\
\hline LFW \\
\hline LFW \\
\hline LFW 34 \\
\hline LFW 34 \\
\hline FW 34 \\
\hline LFW 3 \\
\hline LFW 34 \\
\hline LFW 36 \\
\hline LFW 34 \\
\hline LFW \\
\hline LFW 34 \\
\hline LFW 14 \\
\hline LFW 34 \\
\hline LFW 34 \\
\hline LFW 34 \\
\hline$\frac{10}{4}$ \\
\hline
\end{tabular}




\begin{tabular}{|c|}
\hline Well Hems \\
\hline LFW 34 \\
\hline FW 34 \\
\hline LFW 34 \\
\hline LFW 34 \\
\hline LF 34 \\
\hline LFW \\
\hline$L F W 34$ \\
\hline LWW 34 \\
\hline LFW 34 \\
\hline LFW 34 \\
\hline LFW 34 \\
\hline LFW 34 \\
\hline LF 34 \\
\hline$L F W 34$ \\
\hline LFW34 \\
\hline LFW 4 \\
\hline LFW \\
\hline LFW 34 \\
\hline LFW 34 \\
\hline LFW 34 \\
\hline LFW 34 \\
\hline LW 36 \\
\hline LFW 34 \\
\hline LFW 34 \\
\hline LFW 3A \\
\hline LFW 34 \\
\hline LW 34 \\
\hline FW 34 \\
\hline LFW 34 \\
\hline LFW \\
\hline LFW 34 \\
\hline LFW3i \\
\hline LFW34 \\
\hline FW 34 \\
\hline LF 34 \\
\hline LFW 34 \\
\hline $\mathrm{LFW}^{34}$ \\
\hline LFW 34 \\
\hline IFW 34 \\
\hline LFW 34 \\
\hline LFW 34 \\
\hline LFW 34 \\
\hline LFW 34 \\
\hline LFW 34 \\
\hline FW 34 \\
\hline LFW 30 \\
\hline LFW 34 \\
\hline LFW 34 \\
\hline LFW 34 \\
\hline LFW 34 \\
\hline LFW 3 \\
\hline LFW \\
\hline LFW 34 \\
\hline LFW 34 \\
\hline LFW 34 \\
\hline LFW \\
\hline LFW34 \\
\hline LFW 34 \\
\hline LFW 34 \\
\hline LFW 34 \\
\hline LFW 34 \\
\hline$L F W 3$ \\
\hline LFW 34 \\
\hline LFW 34 \\
\hline LFW 34 \\
\hline LFW 34 \\
\hline LF 34 \\
\hline LFW 34 \\
\hline LFW 3 \\
\hline LFW 34 \\
\hline LFW 34 \\
\hline FW 34 \\
\hline LFW 34 \\
\hline LFW 34 \\
\hline LFW 3 \\
\hline LFW 34 \\
\hline FW 34 \\
\hline LFW 34 \\
\hline LFW \\
\hline LFW 34 \\
\hline LFW \\
\hline LFW 34 \\
\hline LFW34 \\
\hline LFW 34 \\
\hline LFW 430 \\
\hline LF+38 \\
\hline$L F W \triangle 136$ \\
\hline LFW 438 \\
\hline$\angle F W 430$ \\
\hline LFW 438 \\
\hline LW 438 \\
\hline LFW 430 \\
\hline LFW 430 \\
\hline LFW 430 \\
\hline FW 439 \\
\hline LFW 430 \\
\hline LFW 43. \\
\hline
\end{tabular}




\begin{tabular}{|c|}
\hline Woll Mamo \\
\hline LFW 43B \\
\hline FW 138 \\
\hline LFW 438 \\
\hline FW 438 \\
\hline LFW 438 \\
\hline FW +38 \\
\hline LFW 438 \\
\hline LFW 438 \\
\hline$L F W 438$ \\
\hline LFW 438 \\
\hline LFW 43B \\
\hline LW 438 \\
\hline LFW 438 \\
\hline LFW 438 \\
\hline LFW 438 \\
\hline LFW 438 \\
\hline LFW 430 \\
\hline LFW 438 \\
\hline LFW 438 \\
\hline LFW 438 \\
\hline LF 438 \\
\hline LFW 438 \\
\hline$L F W 438$ \\
\hline LW 438 \\
\hline LFW 436 \\
\hline LFW 438 \\
\hline LFW 438 \\
\hline LFW 439 \\
\hline$F W 438$ \\
\hline LFW 439 \\
\hline FW 438 \\
\hline FW 438 \\
\hline FW 438 \\
\hline LFW 43B \\
\hline LFW 430 \\
\hline LFW 436 \\
\hline LW 438 \\
\hline LW 456 \\
\hline LF 436 \\
\hline$L F W_{430}$ \\
\hline LFW 438 \\
\hline LFW 438 \\
\hline FW 430 \\
\hline FW 438 \\
\hline FW 436 \\
\hline$L F=433^{\circ}$ \\
\hline$F W+39$ \\
\hline $5-W+36$ \\
\hline LWW 436 \\
\hline LFW 438 \\
\hline LFW 438 \\
\hline LFW 438 \\
\hline LFW $43 B$ \\
\hline LFW 438 \\
\hline LFW 438 \\
\hline LFW 438 \\
\hline FW 436 \\
\hline LFW 438 \\
\hline LFW 136 \\
\hline LW 438 \\
\hline$L F W \triangle 36$ \\
\hline LF 438 \\
\hline LFW 438 \\
\hline$[F W+38$ \\
\hline LFW 430 \\
\hline LFW 438 \\
\hline LFW 438 \\
\hline LFW 439 \\
\hline LFW 430 \\
\hline LW 438 \\
\hline LFW 438 \\
\hline LPW 430 \\
\hline LFW 438 \\
\hline$L F W 438$ \\
\hline$L F W+38$ \\
\hline$F W 438$ \\
\hline FW 438 \\
\hline$L F W 038$ \\
\hline LFW \\
\hline LFW 430 \\
\hline LFW \\
\hline LFW 438 \\
\hline LFW 430 \\
\hline L्W 430 \\
\hline $15 W 436$ \\
\hline LW 436 \\
\hline$L F W<30$ \\
\hline $5 \times 430$ \\
\hline LFW 430 \\
\hline$F W 430$ \\
\hline IfW 438 \\
\hline LFW 430 \\
\hline LFW 438 \\
\hline LWW 130 \\
\hline LFW 438 \\
\hline IFW 430 \\
\hline IfW 430 \\
\hline
\end{tabular}




\begin{tabular}{|c|}
\hline Woll Name \\
\hline$L W W$ \\
\hline LPW 430 \\
\hline LFW 438 \\
\hline LFW 4 30 \\
\hline LFW 438 \\
\hline LFW 43B \\
\hline LFW 438 \\
\hline LFW 438 \\
\hline LFW 438 \\
\hline LFW 438 \\
\hline LFW 438 \\
\hline$L F W 438$ \\
\hline LFW 438 \\
\hline$L F W 438$ \\
\hline LFW 438 \\
\hline LFW 438 \\
\hline$L F W 438$ \\
\hline$L F+438$ \\
\hline LFW 438 \\
\hline LFW 438 \\
\hline LFW 438 \\
\hline LFW 438 \\
\hline LFW 438 \\
\hline$L F W 438$ \\
\hline$L F W 438$ \\
\hline LFW 436 \\
\hline LFW 430 \\
\hline LFW 436 \\
\hline LFW 438 \\
\hline LFW 438 \\
\hline LFW 438 \\
\hline fow 438 \\
\hline LFW 43C \\
\hline$L-W 43 C$ \\
\hline LFW $43 \mathrm{C}$ \\
\hline LFW 43C \\
\hline LFW 43C \\
\hline LFW \&3C \\
\hline LFW 43C \\
\hline LFW 43C \\
\hline LF 43C \\
\hline LFW 43C \\
\hline$L F 43 C$ \\
\hline LFW 43C \\
\hline LFW $43 C$ \\
\hline$L F=43 C$ \\
\hline LFW 43C \\
\hline LFW +3C \\
\hline LF 43C \\
\hline LF 4 43C \\
\hline FW 43C \\
\hline LFW 43C \\
\hline LFW 43C \\
\hline LFW 43C \\
\hline LFW $+3 \mathrm{C}$ \\
\hline$L F W 43 C$ \\
\hline LFW $43 \mathrm{C}$ \\
\hline $\mathrm{LFW} 43 \mathrm{C}$ \\
\hline$L F W 43 C$ \\
\hline FW 4,3C \\
\hline FFW 43C \\
\hline LFW $43 C$ \\
\hline FW $43 C$ \\
\hline LFW 43C \\
\hline LFW 43C \\
\hline LFW 43C \\
\hline LFW 43C \\
\hline LFW 4,3C \\
\hline LFW 43C \\
\hline LW $43 \mathrm{C}$ \\
\hline LFW $43 \mathrm{C}$ \\
\hline LFW 43 C \\
\hline LFW 43C \\
\hline$L F+3 C$ \\
\hline FW 43C \\
\hline$L F W 43 C$ \\
\hline$L F W \cdot 3 C$ \\
\hline LFW $43 \mathrm{C}$ \\
\hline LF 43C \\
\hline LF+3C \\
\hline LFW 4JC \\
\hline LFW 43C \\
\hline LF 43C \\
\hline LFW 43C \\
\hline$L F W 43 C$ \\
\hline$L F W \triangle 3 C$ \\
\hline$L F W+3 C$ \\
\hline LFW 43C \\
\hline LFW 43C \\
\hline LFW 43C \\
\hline$L F W 43 C$ \\
\hline LFW $43 \mathrm{C}$ \\
\hline LFW 43C \\
\hline LFW A3C \\
\hline LFW $43 \mathrm{C}$ \\
\hline LF 43C \\
\hline IFW 43C \\
\hline
\end{tabular}




\begin{tabular}{|c|}
\hline Woll Name \\
\hline LFW $\triangle 3 C$ \\
\hline$[F W 43 C$ \\
\hline LW 43C \\
\hline LF:3C \\
\hline$\angle F W$ 43C \\
\hline LFW $43 C$ \\
\hline LW $43 \mathrm{C}$ \\
\hline LFW 43C \\
\hline LFW $43 C$ \\
\hline $\mathrm{LFW} 43 \mathrm{C}$ \\
\hline$\angle F W \& 3 C$ \\
\hline LFW 43C \\
\hline LFW 43C \\
\hline LFW 43C \\
\hline LF $43 C$ \\
\hline LFW $43 \mathrm{C}$ \\
\hline LFW $\triangle 3 C$ \\
\hline LF $43 \mathrm{C}$ \\
\hline FW 43C \\
\hline LFW $43 C$ \\
\hline LFW $43 \mathrm{C}$ \\
\hline$\angle F W 43 C$ \\
\hline LFW 43C \\
\hline LFW 43C \\
\hline LFW $43 C$ \\
\hline LFW 43C \\
\hline FW 43C \\
\hline FW $43 C$ \\
\hline LFW $43 \mathrm{C}$ \\
\hline LFW $43 C$ \\
\hline FW 43C \\
\hline $5 W+3 C$ \\
\hline FW 43C \\
\hline LFW $43 C$ \\
\hline LFW 43C \\
\hline LFW 430 \\
\hline LPW 43C \\
\hline WW $43 C$ \\
\hline LPW $43 C$ \\
\hline FF+3 \\
\hline LFW 43C \\
\hline LFW 43C \\
\hline LFW $43 C$ \\
\hline LFW 43C \\
\hline LFW $43 C$ \\
\hline $1 F W 43 C$ \\
\hline LFW $43 C$ \\
\hline LFW $43 \mathrm{C}$ \\
\hline LFW $43 C$ \\
\hline LFW 43C \\
\hline LFW $43 C$ \\
\hline LF $43 C$ \\
\hline LFW 43C \\
\hline LF 43C \\
\hline LFW $43 C$ \\
\hline LF $43 C$ \\
\hline LFW 43C \\
\hline FF $43 C$ \\
\hline LFW 43C \\
\hline LFW $43 C$ \\
\hline LFW $43 C$ \\
\hline LFW 43C \\
\hline LFW 43C \\
\hline LFW 43C \\
\hline LFW 43C \\
\hline LFW $43 C$ \\
\hline$L F W 43 C$ \\
\hline$\overline{L F W} \mathbf{4 3 C}$ \\
\hline LFW A3C \\
\hline LFW 43C \\
\hline FW 43C \\
\hline LFW $43 \mathrm{C}$ \\
\hline LFW 43C \\
\hline LFW $43 C$ \\
\hline$F W+3 C$ \\
\hline LFW $43 \mathrm{C}$ \\
\hline$L F+3 C$ \\
\hline LFW 430 \\
\hline LFW 430 \\
\hline LFW 430 \\
\hline LFW 430 \\
\hline LFW 430 \\
\hline LW 430 \\
\hline $5 \times W 430$ \\
\hline LW 430 \\
\hline LFW 430 \\
\hline LFW 430 \\
\hline LFW 430 \\
\hline FW 430 \\
\hline$F=6,30$ \\
\hline LFW 430 \\
\hline $5 F W+30$ \\
\hline$L F W+30$ \\
\hline UFW 430 \\
\hline FW 430 \\
\hline FW 430 \\
\hline LFWa30 \\
\hline
\end{tabular}




\begin{tabular}{|c|}
\hline Woll Name \\
\hline LFW 430 \\
\hline LFW 430 \\
\hline LFW 430 \\
\hline$L F W 430$ \\
\hline LFW 430 \\
\hline LFW 430 \\
\hline LFW 430 \\
\hline LFW 430 \\
\hline$\angle F W 430$ \\
\hline LFW 430 \\
\hline LFW 130 \\
\hline LFW 430 \\
\hline$L F+30$ \\
\hline$L F+30$ \\
\hline LFW 430 \\
\hline LFW 430 \\
\hline LFW 430 \\
\hline LFW 430 \\
\hline WW 430 \\
\hline$L F W \triangle 30$ \\
\hline LF 430 \\
\hline LFW 430 \\
\hline LFW 430 \\
\hline$\angle F W 430$ \\
\hline$\angle F W 430$ \\
\hline FW 430 \\
\hline LW 430 \\
\hline LFW 430 \\
\hline LF 430 \\
\hline $1 F W 430$ \\
\hline $4 F 430$ \\
\hline LFW 430 \\
\hline LW 430 \\
\hline FW 430 \\
\hline$\angle F=430$ \\
\hline FW 430 \\
\hline LFW 430 \\
\hline LF 430 \\
\hline $1 F W 430$ \\
\hline$L F W 430$ \\
\hline LFW 430 \\
\hline FW 430 \\
\hline FW 430 \\
\hline FFW 130 \\
\hline LW 430 \\
\hline LF 430 \\
\hline FW 430 \\
\hline$L F+30$ \\
\hline LFW 430 \\
\hline LFW 430 \\
\hline LFW 430 \\
\hline LFW 430 \\
\hline fFW 430 \\
\hline FW 430 \\
\hline LFW.430 \\
\hline LFW 430 \\
\hline LFW 430 \\
\hline$L F W 430$ \\
\hline LFW 74C \\
\hline LWT 4 CC \\
\hline LFW T4C \\
\hline LFWTC \\
\hline LFW T4C \\
\hline LFW 74C \\
\hline LFWTC \\
\hline LFW TAC \\
\hline LW74C \\
\hline FW 74C \\
\hline LW74C \\
\hline LFW7C \\
\hline LW $74 \mathrm{C}$ \\
\hline LW74C \\
\hline LFWTAC \\
\hline LFW 74C \\
\hline LFW TAC \\
\hline LFW TAC \\
\hline FW $74 \mathrm{C}$ \\
\hline FW74C \\
\hline LF 7\&C \\
\hline LFTIC \\
\hline FWTC \\
\hline LFW T\&C \\
\hline LW74C \\
\hline LFW T\&C \\
\hline LF TAC \\
\hline LFW TLC \\
\hline LFW 74C \\
\hline LFW 7AC \\
\hline FW 74C \\
\hline LFW 74C \\
\hline LFW \\
\hline LFW 74 \\
\hline LWTAC \\
\hline LFW 74C \\
\hline LWTSC \\
\hline LW 74C \\
\hline LFWTAC \\
\hline
\end{tabular}




\begin{tabular}{|c|}
\hline Woll Name \\
\hline LFW T4C \\
\hline LFW 74C \\
\hline LFW TSC \\
\hline LFW 74C \\
\hline LFW 7aC \\
\hline LFW 74C \\
\hline LFW TAC \\
\hline FW $74 \mathrm{C}$ \\
\hline LFW 74C \\
\hline LFW TAC \\
\hline LFW 74C \\
\hline LFW 74C \\
\hline LFW 74C \\
\hline LFW 74C \\
\hline LFW T4C \\
\hline LFW 74C \\
\hline LFW TAC \\
\hline LFW TAC \\
\hline FW $74 \mathrm{C}$ \\
\hline LF $74 C$ \\
\hline LFW TAC \\
\hline LFW 74C \\
\hline IFW $74 \mathrm{C}$ \\
\hline LFT TSC \\
\hline FWTAC \\
\hline LFW TAC \\
\hline LFW TAC \\
\hline LFW7QC \\
\hline LFW 74C \\
\hline LFW 74C \\
\hline LFW 74C \\
\hline LFW T4C \\
\hline LFWTSC \\
\hline LFW 74C \\
\hline LFW 74C \\
\hline LFWT4C \\
\hline LFW TAC \\
\hline LFWTAC \\
\hline LFW TAC \\
\hline LFW T\&C \\
\hline IFWT4C \\
\hline LFW TAC \\
\hline LFW 74C \\
\hline LFW74C \\
\hline FWTAC \\
\hline LFW 74 \\
\hline LFW74C \\
\hline LFWTC \\
\hline FF 74C \\
\hline IFW7AC \\
\hline FW 74C \\
\hline LFWTAC \\
\hline LFW $74 \mathrm{C}$ \\
\hline LFW 74C \\
\hline LFW 7AC \\
\hline LFW 74C \\
\hline LPW TAC \\
\hline LFW 74C \\
\hline LFWTIC \\
\hline LFWTAC \\
\hline LFW TAC \\
\hline LFW 74C \\
\hline LFW 74C \\
\hline LFW 74C \\
\hline LFW TIC \\
\hline LFW 74C \\
\hline LFW 7SC \\
\hline LFW74C \\
\hline LFW 74C \\
\hline LFWTC \\
\hline LFWTAC \\
\hline LFW TAC \\
\hline LFW 74C \\
\hline LFW \\
\hline LFWTac \\
\hline LFW74C \\
\hline LFW 74C \\
\hline LFW 74C \\
\hline LFW TAC \\
\hline LFW TAC \\
\hline LFW74C \\
\hline LFWTC \\
\hline LFW TAC \\
\hline SW74C \\
\hline LFW $74 C$ \\
\hline LFW $74 C$ \\
\hline LFWTAC \\
\hline LFT IC \\
\hline FW 74C \\
\hline LFW7CC \\
\hline IFW 74C \\
\hline LFW74C \\
\hline LFWTAC \\
\hline LFWTAC \\
\hline LFW 74C \\
\hline LFW74C \\
\hline LFW T\&C \\
\hline
\end{tabular}




\begin{tabular}{|c|}
\hline Woll Namo \\
\hline LFW 74C \\
\hline LFW T4C \\
\hline LFWTAC \\
\hline LFW74C \\
\hline LFW 7AC \\
\hline WWT4C \\
\hline FWTAC \\
\hline LFW 74C \\
\hline FW74C \\
\hline LFW T4C \\
\hline LFW 74C \\
\hline LFW $74 C$ \\
\hline LFW 7AC \\
\hline LFW TLC \\
\hline LFW TAC \\
\hline LFW T4C \\
\hline LFWTSC \\
\hline LFW 74C \\
\hline LFW T4C \\
\hline LFW TSC \\
\hline LFWTAC \\
\hline LFW T4C \\
\hline LFWTAC \\
\hline LFW TAC \\
\hline LFW 74C \\
\hline LFW 74C \\
\hline LFW 74C \\
\hline LFW 7\&C \\
\hline LFW 7AC \\
\hline LFW 74C \\
\hline LFW 74C \\
\hline LFW 7\&C \\
\hline LFW TAC \\
\hline LFW $\$ \& C$ \\
\hline LFW 74C \\
\hline LFW 7AC \\
\hline LF $74 C$ \\
\hline LFW 74C \\
\hline LFW 74C \\
\hline LF 74C \\
\hline LFW 74C \\
\hline FWT4C \\
\hline FW 74C \\
\hline LFW 74C \\
\hline LFWTAC \\
\hline LFW TLC \\
\hline LF $74 C$ \\
\hline LF 74C \\
\hline LFW 74E \\
\hline LFW 74C \\
\hline LFW 74C \\
\hline LFWTAC \\
\hline LFW 74C \\
\hline LFWTAC \\
\hline LFWTAC \\
\hline LW 74C \\
\hline LWTIC \\
\hline LFW $74 C$ \\
\hline LFW 74C \\
\hline LFW74C \\
\hline LFW TSC \\
\hline LFW 74C \\
\hline LFW 74C \\
\hline LFW 7\&C \\
\hline LFW THC \\
\hline LFW TLC \\
\hline FWTAC \\
\hline LFW T4C \\
\hline LFW 740 \\
\hline LFW 740 \\
\hline LFW 740 \\
\hline LFW740 \\
\hline LFW 740 \\
\hline LFW 740 \\
\hline$L=740$ \\
\hline LFW 700 \\
\hline LFW 740 \\
\hline LFW 740 \\
\hline LW 740 \\
\hline LFW 740 \\
\hline LFW 740 \\
\hline LW 740 \\
\hline LFW 740 \\
\hline LFW 740 \\
\hline LFW 740 \\
\hline LFW 740 \\
\hline LFW 740 \\
\hline LFW 740 \\
\hline LFW 740 \\
\hline UF 740 \\
\hline LFW 740 \\
\hline LFW 740 \\
\hline LFW 740 \\
\hline LFW 740 \\
\hline LW 740 \\
\hline LFW 740 \\
\hline LFW740 \\
\hline
\end{tabular}




\begin{tabular}{|c|}
\hline Woll Name \\
\hline LFW 740 \\
\hline LFW 740 \\
\hline LFW 740 \\
\hline LFWT4O \\
\hline LFW 740 \\
\hline LFW 740 \\
\hline LFW 740 \\
\hline LFW 740 \\
\hline FW740 \\
\hline LFW 740 \\
\hline LFW 740 \\
\hline $5 F 740$ \\
\hline LFW780 \\
\hline LFW740 \\
\hline LFW 740 \\
\hline LF 740 \\
\hline LFW T40 \\
\hline LFW710 \\
\hline LWW740 \\
\hline LFW 760 \\
\hline LFW740 \\
\hline$L F$ W740 \\
\hline LFW740 \\
\hline $5 \times 740$ \\
\hline LFW 740 \\
\hline LFW740 \\
\hline LFW 740 \\
\hline LFW 740 \\
\hline LFW740 \\
\hline LFW 740 \\
\hline LFW 740 \\
\hline LFW 700 \\
\hline FW 780 \\
\hline$L F W 740$ \\
\hline LFW 710 \\
\hline LW 740 \\
\hline LFW 740 \\
\hline LFW 740 \\
\hline LFW 740 \\
\hline LFW740 \\
\hline LFW 740 \\
\hline LFW 740 \\
\hline LFW 740 \\
\hline LFWT40 \\
\hline LFW740 \\
\hline LFW 740 \\
\hline LFW740 \\
\hline LF 740 \\
\hline LFW740 \\
\hline LFW 740 \\
\hline LFW740 \\
\hline LFW740 \\
\hline LFW 740 \\
\hline Fow 740 \\
\hline LF 740 \\
\hline LFW 740 \\
\hline LFW 740 \\
\hline LFW740 \\
\hline FW 740 \\
\hline IFW 740 \\
\hline FW 740 \\
\hline LFW740 \\
\hline LFW 740 \\
\hline LFW74D \\
\hline LFW 740 \\
\hline FW 740 \\
\hline LFW 740 \\
\hline LFW 140 \\
\hline LFW 740 \\
\hline LFW 740 \\
\hline FW 740 \\
\hline LFW 740 \\
\hline LPW 740 \\
\hline LFW 740 \\
\hline LFW 740 \\
\hline LFW740 \\
\hline LFW 740 \\
\hline LFW740 \\
\hline $1 F W 740$ \\
\hline LFW 740 \\
\hline LFW 740 \\
\hline LFW TAD \\
\hline LFW74D \\
\hline LF 740 \\
\hline LF 740 \\
\hline LW 740 \\
\hline LW740 \\
\hline LFW 740 \\
\hline LFW 740 \\
\hline LFW 740 \\
\hline LFW 740 \\
\hline LFW 740 \\
\hline LF 740 \\
\hline LFW 740 \\
\hline LW740 \\
\hline IFW 740 \\
\hline LFW \\
\hline
\end{tabular}




\begin{tabular}{|c|}
\hline $\begin{array}{l}\text { Well rome } \\
\text { LF 740 }\end{array}$ \\
\hline \\
\hline LFW 740 \\
\hline LFW 740 \\
\hline LFW 740 \\
\hline LFW 740 \\
\hline LFW 740 \\
\hline LFW 740 \\
\hline LFW 740 \\
\hline LFW 740 \\
\hline LFW 740 \\
\hline LFW 740 \\
\hline LFW 740 \\
\hline FW 740 \\
\hline LFW $7 \$ 0$ \\
\hline LFW 740 \\
\hline LF 740 \\
\hline LFT140 \\
\hline LFW 740 \\
\hline LFW 740 \\
\hline LFW 740 \\
\hline LFW 740 \\
\hline LFW 740 \\
\hline LFW 740 \\
\hline LFW 740 \\
\hline LFW 740 \\
\hline FW 740 \\
\hline LFW 740 \\
\hline LFW 740 \\
\hline LFW 740 \\
\hline LFW 740 \\
\hline LFW 740 \\
\hline LFW 740 \\
\hline LFW 740 \\
\hline LFW 740 \\
\hline LFW 740 \\
\hline FW 740 \\
\hline LFW740 \\
\hline LFW 740 \\
\hline FW740 \\
\hline LFW 740 \\
\hline LFW 740 \\
\hline LFW 740 \\
\hline LFW740 \\
\hline FW740 \\
\hline LFW 740 \\
\hline LW 740 \\
\hline LFW 740 \\
\hline LF 740 \\
\hline LFW 740 \\
\hline FW 740 \\
\hline LWW 740 \\
\hline LFW 740 \\
\hline LW 740 \\
\hline LFT \\
\hline LW740 \\
\hline LFW740 \\
\hline FW 740 \\
\hline FW740 \\
\hline LWW 740 \\
\hline LFW 740 \\
\hline LFW 740 \\
\hline$L F 740$ \\
\hline LFW 740 \\
\hline LFW 710 \\
\hline $5 \times 740$ \\
\hline LFW740 \\
\hline FW 740 \\
\hline LFW740 \\
\hline LFW740 \\
\hline LFW740 \\
\hline$L F W 760$ \\
\hline LFW 740 \\
\hline LF 740 \\
\hline LFW 740 \\
\hline LFW 740 \\
\hline LFW 740 \\
\hline LFW 740 \\
\hline LFW 740 \\
\hline LFW TSC \\
\hline LFW7SC \\
\hline SFTSC \\
\hline LFW $75 \mathrm{C}$ \\
\hline LFW TSC \\
\hline LFW 7SC \\
\hline LFW 7SC \\
\hline LFW $75 \mathrm{C}$ \\
\hline FWTSC \\
\hline FW 7SC \\
\hline FW 7SC \\
\hline LFW 7SC \\
\hline FWTSC \\
\hline LFW TSC \\
\hline FW 75 \\
\hline LFW $75 C$ \\
\hline LFW TSC \\
\hline LFW 75 \\
\hline LFWTSC \\
\hline
\end{tabular}




\begin{tabular}{|c|}
\hline Woll Nome \\
\hline LFW $75 C$ \\
\hline FW 73C \\
\hline LFW 75C \\
\hline LFW 756 \\
\hline LFW 73C \\
\hline LFW7SC \\
\hline LFW $75 C$ \\
\hline LFW 75C \\
\hline LFW $75 C$ \\
\hline LFW 7SC \\
\hline LFW $75 C$ \\
\hline LFW $75 \mathrm{C}$ \\
\hline LFWTSC \\
\hline LFW 75C \\
\hline FW 75C \\
\hline LFWTSC \\
\hline LFWTSC \\
\hline LFW 750 \\
\hline LFW 7SC \\
\hline FW $75 C$ \\
\hline LFW 75C \\
\hline LW7SC \\
\hline LW 7SC \\
\hline LWW $75 C$ \\
\hline LFW TSC \\
\hline LFW 75C \\
\hline LFW TSC \\
\hline LFW TSC \\
\hline LFW 7SC \\
\hline LFW 75C \\
\hline LFW 75C \\
\hline LFW TSC \\
\hline LFW $75 C$ \\
\hline LF \\
\hline LFW $75 C$ \\
\hline LFW 75C \\
\hline LFW 7SC \\
\hline LFW 7SC \\
\hline LFW75C \\
\hline LFW 7SC \\
\hline LFW 7SC \\
\hline IFW 7SC \\
\hline LFW \\
\hline FW $75 C$ \\
\hline LFW $75 C$ \\
\hline LFW $73 C$ \\
\hline LW 7SC \\
\hline LF-73c \\
\hline LFW TSC \\
\hline FWTBC \\
\hline LFW 7SC \\
\hline LFW TSC \\
\hline FWTSC \\
\hline LFW TSC \\
\hline LFW 7SC \\
\hline LF $75 C$ \\
\hline FW 756 \\
\hline LFW 7SC \\
\hline LWW $75 C$ \\
\hline FW 75C \\
\hline IfW TSC \\
\hline LFW 7SC \\
\hline FW 7SC \\
\hline FWTSC \\
\hline LFW $75 C$ \\
\hline LFW 7SC \\
\hline LFW 7SC \\
\hline LFW TSC \\
\hline LFW 7SC \\
\hline LFW TSC \\
\hline LWTBC \\
\hline LFW 750 \\
\hline LFW TSC \\
\hline LFW75C \\
\hline LFW 7SC \\
\hline LFW $75 \mathrm{C}$ \\
\hline LFW 7SC \\
\hline LFW 75C \\
\hline LFW 7SC \\
\hline FWTSC \\
\hline LFW $75 C$ \\
\hline LFW 75C \\
\hline LFW 7SC \\
\hline FW $75 C$ \\
\hline LF $75 C$ \\
\hline LWW $7 \bar{C}$ \\
\hline LFW 7SC \\
\hline LFW $75 C$ \\
\hline LFW TSC \\
\hline LFW $75 C$ \\
\hline LFW TSC \\
\hline LW TSC \\
\hline LW 7SC \\
\hline LWTSC \\
\hline LFW TSC \\
\hline LF TSC \\
\hline LFW 7SC \\
\hline
\end{tabular}




\begin{tabular}{|c|}
\hline $\begin{array}{l}\text { Woll Nemm } \\
\text { LFW } 7 \text { SCC }\end{array}$ \\
\hline LFW 7SC \\
\hline LFW7SC \\
\hline LFW \\
\hline FW 7SC \\
\hline LFW 7SC \\
\hline LFW 7SC \\
\hline LFW $75 C$ \\
\hline LFW TSC \\
\hline LFW $75 C$ \\
\hline LFW 7SC \\
\hline LFW $75 C$ \\
\hline LFW TSC \\
\hline LFW \\
\hline FW $73 C$ \\
\hline LFW 7SC \\
\hline LFW 7SC \\
\hline LFW 7SC \\
\hline LFW 7SC \\
\hline LFW 7SC \\
\hline LFW TSC \\
\hline LFW TSC \\
\hline LFW 7SC \\
\hline LWW 73C \\
\hline LFW 7 SC \\
\hline LFW 7SC \\
\hline LFW TSC \\
\hline LFW75C \\
\hline LFW TSC \\
\hline$L F W 7 \overline{S C}$ \\
\hline LFW TSC \\
\hline LFW 7SC \\
\hline LFW TSC \\
\hline LFW TSC \\
\hline LFW 7SC \\
\hline LFW TSC \\
\hline LFW $75 C$ \\
\hline LFW 7SC \\
\hline FW 7 S $^{\circ}$ \\
\hline LFW 7SC \\
\hline LFW TSC \\
\hline LFW TSC \\
\hline LFW 7SC \\
\hline LFW 7SC \\
\hline LFW 75C \\
\hline$L F W 7 S C$ \\
\hline LFW $75 \mathrm{C}$ \\
\hline LFW \\
\hline LFW $75 C$ \\
\hline LFW TSC \\
\hline LFW TBC \\
\hline LFW 7SC \\
\hline LFW TSC \\
\hline LFW 75C \\
\hline UFW 7SC \\
\hline LFW $75 C$ \\
\hline LFW 7SC \\
\hline LFW TSC \\
\hline LFW 7SC \\
\hline LFW 7SC \\
\hline LFW 75C \\
\hline LFW 7SC \\
\hline LFW $75 C$ \\
\hline LFW TSC \\
\hline LFW 7SC \\
\hline LW 7SC \\
\hline LFW 7SC \\
\hline LFW TSC \\
\hline LFWTSC \\
\hline LFW TSC \\
\hline LFW 7SC \\
\hline LFW 7SC \\
\hline LFW 7 SC \\
\hline LFW 7SC \\
\hline LFW $75 C$ \\
\hline LFW $7 S C$ \\
\hline LFW $75 \mathrm{C}$ \\
\hline LFW 7SC \\
\hline LFW TSC \\
\hline LFWTSC \\
\hline LFW TSC \\
\hline LFW \\
\hline LFW 7SC \\
\hline LFW $75 C$ \\
\hline LWTSC \\
\hline LFW $75 \mathrm{C}$ \\
\hline LFW 7SC \\
\hline LFW 75 \\
\hline LFW 756 \\
\hline LF \\
\hline LFW 7SC \\
\hline LFW TSC \\
\hline FW TSC \\
\hline LFW 7SC \\
\hline LFW 7SC \\
\hline LEW TSC \\
\hline LFW TSC \\
\hline LFW TSC \\
\hline
\end{tabular}




\begin{tabular}{|c|}
\hline Well Name \\
\hline LFW $75 C$ \\
\hline FW $75 C$ \\
\hline LFW TSC \\
\hline FW TSC \\
\hline LFW 7SC \\
\hline LFW TSC \\
\hline LFW $75 \mathrm{C}$ \\
\hline LFW 75C \\
\hline LFW 75C \\
\hline LFW75C \\
\hline LFW 7SC \\
\hline LFW 7SC \\
\hline LFW 75C \\
\hline FW $75 \mathrm{C}$ \\
\hline LWTSC \\
\hline LFW $75 C$ \\
\hline LFW TSC \\
\hline LFW TSC \\
\hline LFW $75 C$ \\
\hline IFW 75C \\
\hline LFW TSC \\
\hline LFW73C \\
\hline LFW TSC \\
\hline LFW 75C \\
\hline LFW $75 \mathrm{C}$ \\
\hline FW $75 C$ \\
\hline LFW 750 \\
\hline LFW 7SC \\
\hline LFW 75C \\
\hline FW 75C \\
\hline LFW 7SC \\
\hline LFW $75 \mathrm{C}$ \\
\hline IFW TSC \\
\hline FW $75 \mathrm{C}$ \\
\hline LFW TSC \\
\hline LFW $75 \mathrm{C}$ \\
\hline LFW 7SC \\
\hline LFW $75 \mathrm{C}$ \\
\hline LW 7SC \\
\hline IFW $75 C$ \\
\hline FW $75 \mathrm{C}$ \\
\hline LF $75 C$ \\
\hline LFW $75 C$ \\
\hline fow $75 \mathrm{C}$ \\
\hline LFWTSC \\
\hline LFW $75 \mathrm{C}$ \\
\hline FW $75 C$ \\
\hline FW $75 C$ \\
\hline LFW $75 C$ \\
\hline FW7SC \\
\hline LFW $73 C$ \\
\hline UF $75 C$ \\
\hline LFT \\
\hline LFW $75 \mathrm{C}$ \\
\hline LFW 75C \\
\hline LFW $75 \mathrm{C}$ \\
\hline LFW TSC \\
\hline LW 750 \\
\hline LFW $75 \mathrm{C}$ \\
\hline FWTSC \\
\hline LFW 7 SSC \\
\hline LFW 7SC \\
\hline LFW $73 C$ \\
\hline LFW TSC \\
\hline LFW 7SC \\
\hline LFW TSC \\
\hline LFW 75C \\
\hline LFW 7SC \\
\hline FFW 7BC \\
\hline LW 7SC \\
\hline LFW TSC \\
\hline FW.756 \\
\hline LFW 7SC \\
\hline LFW $75 C$ \\
\hline FW $75 \mathrm{C}$ \\
\hline LFW 7SC \\
\hline LFW 7SC \\
\hline f $W 75 C$ \\
\hline UFW TSC \\
\hline LFW 7SC \\
\hline LF 75C \\
\hline LF 7SC \\
\hline LFW $75 \mathrm{C}$ \\
\hline LFW $75 \mathrm{C}$ \\
\hline LFW 7BC \\
\hline LFW $7 S C$ \\
\hline LFW $75 \mathrm{C}$ \\
\hline LFW 7SC \\
\hline LFW TSC \\
\hline LFW TSC \\
\hline LFW TSC \\
\hline LFW TSC \\
\hline LFW $75 C$ \\
\hline LFW 75C \\
\hline LFW 75C \\
\hline FF 7SE \\
\hline FW $75 C$ \\
\hline
\end{tabular}




\begin{tabular}{|c|}
\hline Wel Name \\
\hline LFW 7SC \\
\hline LFW 7SC \\
\hline LFW $75 \mathrm{C}$ \\
\hline LFW7SC \\
\hline LFW $75 C$ \\
\hline LFW $75 C$ \\
\hline LFW 75C \\
\hline LFW 7SC \\
\hline LFW 7SC \\
\hline LFW7SC \\
\hline FW 7SC \\
\hline LFW TSC \\
\hline LFW TSC \\
\hline FW TBC \\
\hline LFW TSC \\
\hline LFW TSC \\
\hline LFWTBC \\
\hline LFW TSC \\
\hline LFW 7SC \\
\hline LFW $75 C$ \\
\hline LFW 750 \\
\hline FF 75C \\
\hline LFW TSC \\
\hline LFWTSC \\
\hline FWW TSC \\
\hline LFW TSC \\
\hline LFWTSC \\
\hline LFW 730 \\
\hline IFW \\
\hline LFW $75 C$ \\
\hline LFW 75C \\
\hline LFWTSC \\
\hline LFW \\
\hline LF $75 C$ \\
\hline FFW \\
\hline LFW TSC \\
\hline LFW 7SC \\
\hline LFW $7 \mathbf{S C}$ \\
\hline LFW TSC \\
\hline LFW 7SC \\
\hline LFW 7SC \\
\hline LFW $7 S C$ \\
\hline LFW7SC \\
\hline LFW TSE \\
\hline LFTISC \\
\hline LFTSC \\
\hline LF $75 C$ \\
\hline LW75C \\
\hline LFWTSC \\
\hline FW $75 C$ \\
\hline FW $75 C$ \\
\hline LFW $75 C$ \\
\hline LFW \\
\hline LFTSC \\
\hline LFW $75 \mathrm{SC}$ \\
\hline WW $75 C$ \\
\hline LFWTSC \\
\hline LFW 7SC \\
\hline LFW $75 C$ \\
\hline LFW $75 C$ \\
\hline FW 7SC \\
\hline LW $75 C$ \\
\hline LF 75C \\
\hline LFW $73 C$ \\
\hline LFW 7SC \\
\hline LFW $75 \mathrm{C}$ \\
\hline FW $75 \mathrm{C}$ \\
\hline FW 7SC \\
\hline LFW $75 C$ \\
\hline LFW TSC \\
\hline FW $7 S C$ \\
\hline FW \\
\hline LFW TSC \\
\hline$L F W 75 C$ \\
\hline LFW $75 C$ \\
\hline LFWTSC \\
\hline LFW $75 C$ \\
\hline LFW 7SC \\
\hline LWTSC \\
\hline LF TSC \\
\hline LFW TSC \\
\hline LW7SC \\
\hline FW 7SC \\
\hline LFW 7SC \\
\hline LFW 75C \\
\hline LFW 7SC \\
\hline $5 W 75 C$ \\
\hline FW/SC \\
\hline LFW $75 \mathrm{C}$ \\
\hline LFW TSC \\
\hline FW $75 C$ \\
\hline जW TSC \\
\hline LFW $75 C$ \\
\hline LFW $75 C$ \\
\hline LFW 75C \\
\hline LFW7SC \\
\hline frW $75 C$ \\
\hline
\end{tabular}




\begin{tabular}{|c|}
\hline Well Nam \\
\hline LFW 7SC \\
\hline LFW 750 \\
\hline LFW750 \\
\hline$\angle F W 750$ \\
\hline LFW 750 \\
\hline LFW 750 \\
\hline LFW 750 \\
\hline LFW TSO \\
\hline LFW 750 \\
\hline LFW 750 \\
\hline LFW T50 \\
\hline L्W750 \\
\hline EW 750 \\
\hline$\angle F W 750$ \\
\hline LFW 750 \\
\hline LFW730 \\
\hline LFW 750 \\
\hline LFW 750 \\
\hline LFW750 \\
\hline LFW750 \\
\hline LFW 750 \\
\hline LFW 730 \\
\hline LW 750 \\
\hline LFW 750 \\
\hline LFW 750 \\
\hline LFW 750 \\
\hline LW 750 \\
\hline LFW 750 \\
\hline LFW 750 \\
\hline LFW 750 \\
\hline LFW 750 \\
\hline LFW 7SSO \\
\hline LFW 750 \\
\hline LFW 750 \\
\hline LFW 750 \\
\hline LFW 750 \\
\hline LFW 750 \\
\hline LFW750 \\
\hline FW750 \\
\hline LFW 750 \\
\hline LFWTSO \\
\hline LFW 750 \\
\hline LFW 750 \\
\hline LFW 750 \\
\hline LFW 750 \\
\hline LFW 750 \\
\hline LFWJ50 \\
\hline LFW 75D \\
\hline LW750 \\
\hline LFW 750 \\
\hline$L F W 750$ \\
\hline LPW 750 \\
\hline $5 \times 750$ \\
\hline LFW 750 \\
\hline LFW 750 \\
\hline LFW 750 \\
\hline FW750 \\
\hline LFW 750 \\
\hline LW 750 \\
\hline LFW 750 \\
\hline LFW7SD \\
\hline LFW 730 \\
\hline LFW 750 \\
\hline LFW 730 \\
\hline LFW 750 \\
\hline LFW 750 \\
\hline LFW 735 \\
\hline LFW 750 \\
\hline LW750 \\
\hline LFW 730 \\
\hline LFW750 \\
\hline LW \\
\hline LFW 750 \\
\hline FW 750 \\
\hline LFW 750 \\
\hline LFWTSO \\
\hline LFW 750 \\
\hline LW 750 \\
\hline LFW 750 \\
\hline LFW 750 \\
\hline FW750 \\
\hline$F W 750$ \\
\hline FW 750 \\
\hline LFW 750 \\
\hline LFW 730 \\
\hline LFW 750 \\
\hline FW 750 \\
\hline$L F W 750$ \\
\hline LW 750 \\
\hline FW 750 \\
\hline FW 750 \\
\hline FW T5O \\
\hline LFW TSO \\
\hline LFW TSO \\
\hline LFW 750 \\
\hline LW 750 \\
\hline LFW 750 \\
\hline
\end{tabular}




\begin{tabular}{|c|}
\hline Woll Nome \\
\hline LFW 750 \\
\hline LFW 750 \\
\hline LFW 750 \\
\hline LFW 750 \\
\hline LFW 750 \\
\hline LFW 750 \\
\hline LFW 750 \\
\hline LFW 750 \\
\hline LFW 750 \\
\hline LFW 750 \\
\hline LFW 750 \\
\hline LFW 7S0 \\
\hline LFW 750 \\
\hline LFW 750 \\
\hline LFW 750 \\
\hline UFW \\
\hline FFW 750 \\
\hline LFW 750 \\
\hline FW 750 \\
\hline LFW 75D \\
\hline LFW 730 \\
\hline LFW 750 \\
\hline LFW 750 \\
\hline LFW 750 \\
\hline$L F W 750$ \\
\hline LFW \\
\hline LFT30 \\
\hline LFW 730 \\
\hline LFW 750 \\
\hline LFW 750 \\
\hline LFW750 \\
\hline LFW 750 \\
\hline LFW 750 \\
\hline LFW 750 \\
\hline LFW \\
\hline LW 750 \\
\hline $1 F W 750$ \\
\hline LFW750 \\
\hline LFW 750 \\
\hline LFW 75D \\
\hline LFW 750 \\
\hline FW 750 \\
\hline FW 750 \\
\hline LFW 750 \\
\hline LW750 \\
\hline LW 750 \\
\hline LFW $75^{\circ}$ \\
\hline LW 750 \\
\hline LFW 750 \\
\hline$L F T$ T50 \\
\hline LFW 750 \\
\hline LF 750 \\
\hline LFW 750 \\
\hline FW 750 \\
\hline LFW 750 \\
\hline LFW 750 \\
\hline LFW 750 \\
\hline LFW 750 \\
\hline LFW 750 \\
\hline LFW750 \\
\hline FW 750 \\
\hline FW 750 \\
\hline LFW 750 \\
\hline LFW 750 \\
\hline LFW 750 \\
\hline LFW 750 \\
\hline LFW 750 \\
\hline LFW 750 \\
\hline LFW 7SD \\
\hline LFW 750 \\
\hline FW 750 \\
\hline LW 750 \\
\hline LFW 730 \\
\hline$\angle F W 730$ \\
\hline LFW 750 \\
\hline LFW 750 \\
\hline LW750 \\
\hline LW 730 \\
\hline LFW TSO \\
\hline LFW 750 \\
\hline FW 750 \\
\hline LFW750 \\
\hline FW 750 \\
\hline LFW 750 \\
\hline LW 750 \\
\hline LFWTSO \\
\hline LFW 730 \\
\hline LFW 750 \\
\hline LFW 750 \\
\hline$L F W 750$ \\
\hline LF 750 \\
\hline FW 750 \\
\hline LFW 750 \\
\hline LW 750 \\
\hline LFW750 \\
\hline LFW 750 \\
\hline LิFW 750 \\
\hline
\end{tabular}




\begin{tabular}{|c|}
\hline Wefl Nam \\
\hline LFW 750 \\
\hline LFW 750 \\
\hline LFW 750 \\
\hline LFW 750 \\
\hline LFW 750 \\
\hline LFW 750 \\
\hline LFW 750 \\
\hline LFW 750 \\
\hline LFW 750 \\
\hline LW 750 \\
\hline LFW 750 \\
\hline LFW 78 \\
\hline LFW 76 \\
\hline FF 76 \\
\hline LFW 78 \\
\hline LFW 76 \\
\hline LFW 76 \\
\hline LFW70 \\
\hline LFW 70 \\
\hline LFW 76 \\
\hline LFW 78 \\
\hline LFW 78 \\
\hline LFW 78 \\
\hline LFW 70 \\
\hline LFW 70 \\
\hline LFW 70 \\
\hline LFW 78 \\
\hline LFW 70 \\
\hline LFW70 \\
\hline LFW 70 \\
\hline LFW TO \\
\hline LFW 70 \\
\hline LFW 70 \\
\hline LW70 \\
\hline LFW 78 \\
\hline FW76 \\
\hline LFF 70 \\
\hline 15W70 \\
\hline LW 70 \\
\hline LFW76 \\
\hline LFW 76 \\
\hline LFW T6 \\
\hline LFW78 \\
\hline LFW76 \\
\hline LFW76 \\
\hline LFW70 \\
\hline LFW76 \\
\hline LFW 70 \\
\hline LFw 78 \\
\hline FWT \\
\hline LFT 70 \\
\hline LFW70 \\
\hline LF 76 \\
\hline LFW 70 \\
\hline LFW 76 \\
\hline LFW 76 \\
\hline LW 76 \\
\hline LFW 78 \\
\hline LFWTO \\
\hline LFW TO \\
\hline LFW 70 \\
\hline LFW70 \\
\hline LFW 78 \\
\hline LFW 78 \\
\hline LFW 78 \\
\hline LFW 78 \\
\hline LFW 78 \\
\hline LFW 76 \\
\hline LFW76 \\
\hline LFW 78 \\
\hline LF 70 \\
\hline LFW 70 \\
\hline LFW 70 \\
\hline LFW 70 \\
\hline FW 76 \\
\hline LF 70 \\
\hline LFW 76 \\
\hline LFW 78 \\
\hline LF 7 \\
\hline LFW \\
\hline LFW 76 \\
\hline LFW 78 \\
\hline LFWT \\
\hline LFW \\
\hline LFW 70 \\
\hline LFW 76 \\
\hline LFW 76 \\
\hline LFW 78 \\
\hline LFW70 \\
\hline LFWT \\
\hline LFW78 \\
\hline LFW70 \\
\hline LFW 76 \\
\hline ثfW 76 \\
\hline UW76 \\
\hline LFWT \\
\hline LFW 76 \\
\hline
\end{tabular}




\begin{tabular}{|c|}
\hline 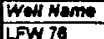 \\
\hline LFW 76 \\
\hline FW 76 \\
\hline LW 78 \\
\hline LFW76 \\
\hline LFW 78 \\
\hline LFW 70 \\
\hline LW7 \\
\hline LFW 76 \\
\hline LFW76 \\
\hline LFW 78 \\
\hline LFW70 \\
\hline LFW 70 \\
\hline LFW 76 \\
\hline LFW76 \\
\hline LFW 76 \\
\hline LFW 76 \\
\hline LFW 78 \\
\hline LFW78 \\
\hline LFW76 \\
\hline LFW 76 \\
\hline LFW78 \\
\hline LFW 70 \\
\hline LFW70 \\
\hline LFW 70 \\
\hline LFW70 \\
\hline LFW 70 \\
\hline LW76 \\
\hline LW79 \\
\hline LFW79 \\
\hline fw70 \\
\hline FW 70 \\
\hline LFW70 \\
\hline LFW \\
\hline LFW 76 \\
\hline LW70 \\
\hline LFW70 \\
\hline LFW70 \\
\hline LFW76 \\
\hline LFW 70 \\
\hline LFW 70 \\
\hline LF 70 \\
\hline LFW 76 \\
\hline LWT \\
\hline FW76 \\
\hline LFTo \\
\hline LFW 78 \\
\hline LFW 7 \\
\hline LFW 78 \\
\hline LWT \\
\hline LFW 70 \\
\hline LFW70 \\
\hline LF 79 \\
\hline LFW70 \\
\hline LF 76 \\
\hline LFW 70 \\
\hline LFW78 \\
\hline LFW 78 \\
\hline LFW 76 \\
\hline LFW 76 \\
\hline LFW 78 \\
\hline LFW 76 \\
\hline LFW76 \\
\hline LF 76 \\
\hline LFW76 \\
\hline LFW 76 \\
\hline LFW 78 \\
\hline LFW 78 \\
\hline LFW78 \\
\hline LFW 76 \\
\hline FW 78 \\
\hline LFW 76 \\
\hline IF 70 \\
\hline LFW \\
\hline $5 \times 70$ \\
\hline $5 \% 70$ \\
\hline LFW 76 \\
\hline LW \\
\hline LFW 76 \\
\hline LW70 \\
\hline LFW 76 \\
\hline LFW 75 \\
\hline LFT \\
\hline WW76 \\
\hline LFW \\
\hline LF 76 \\
\hline LFW 76 \\
\hline LFW 76 \\
\hline LFW 76 \\
\hline LFW7 \\
\hline LFW 76 \\
\hline LFW 76 \\
\hline LFW \\
\hline LFW 76 \\
\hline LFW78 \\
\hline LWTE \\
\hline LF \\
\hline LFT \\
\hline
\end{tabular}




\begin{tabular}{|c|}
\hline Well Name \\
\hline LPW 76 \\
\hline LFW 75 \\
\hline LFW 76 \\
\hline LFW 76 \\
\hline LFW 78 \\
\hline LFW 76 \\
\hline LFW 76 \\
\hline LFW 76 \\
\hline LFW 76 \\
\hline LFW 78 \\
\hline LFW Te \\
\hline LFW 76 \\
\hline LFW 76 \\
\hline LFW 76 \\
\hline LFW 70 \\
\hline LFW 70 \\
\hline LFW 78 \\
\hline LFW 76 \\
\hline LFW76 \\
\hline LFW 78 \\
\hline LFW 78 \\
\hline FWT \\
\hline LFW77 \\
\hline LFW \\
\hline LFWT \\
\hline LFWn \\
\hline LFWT \\
\hline LFWT7 \\
\hline LFWT \\
\hline LWT \\
\hline LFWT \\
\hline FWT \\
\hline LFW77 \\
\hline LWT \\
\hline LFW 77 \\
\hline LFW 77 \\
\hline LFWT7 \\
\hline LW $\pi$ \\
\hline LFW \\
\hline LFWT \\
\hline LFW \\
\hline LFWT \\
\hline LFWT \\
\hline LFWT \\
\hline LFW \\
\hline LFWT \\
\hline LFT \\
\hline tFW \\
\hline LWT \\
\hline LWW \\
\hline LFW \\
\hline town \\
\hline FWn \\
\hline LFw \\
\hline fown \\
\hline LFW $\pi$ \\
\hline LFW \\
\hline LFW 77 \\
\hline LFW7 \\
\hline LFW \\
\hline LFWT \\
\hline LFW77 \\
\hline LFWT \\
\hline LFWT \\
\hline LFW \\
\hline LFW $\pi$ \\
\hline LFW \\
\hline LFW $\pi$ \\
\hline LFW77 \\
\hline INW \\
\hline LFW $\pi$ \\
\hline LFW \\
\hline LFWT \\
\hline LFWT \\
\hline LFW \\
\hline LFW \\
\hline LFW 77 \\
\hline LWT \\
\hline LFWT \\
\hline LFW 77 \\
\hline LFwn \\
\hline LFW \\
\hline fwn \\
\hline जF \\
\hline LFn \\
\hline LFWT \\
\hline LFW $\pi$ \\
\hline LFWT \\
\hline LWT \\
\hline LFWT7 \\
\hline LWT \\
\hline LFWT \\
\hline LFT \\
\hline LFW \\
\hline GWT \\
\hline twT \\
\hline twW \\
\hline
\end{tabular}




\begin{tabular}{|c|c|c|c|c|}
\hline $\begin{array}{l}\text { Well Nom } \\
\text { SW } 78\end{array}$ & $\frac{\text { Sample onts }}{\text { B1198 }}$ & Tastrame & Detection Lint ;RQ & Resuft Unte: \\
\hline LFW 78 & 81198 & 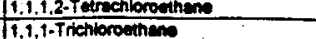 & $\frac{S 10}{510}$ & $\frac{5 \log 2}{5 \log 2}$ \\
\hline LFW 78 & $\$ 1198$ & $1,1,2,2$. Totrectionothen & 5 Siv. & $\frac{100}{\operatorname{sing} 2}$ \\
\hline LFW78 & 81106 & 1,1,2-Trichiopomentere & 5ाU & 5!ugh \\
\hline IFW 78 & 81198 & (1, 1-Dicnlorowthane & siv & $5 \log 2$ \\
\hline LFW 78 & 81198 & 1.1.Dictlopoethylome & So & $5: \log$ \\
\hline LFW78 & 81198 & $1,2,3$ - Trichloropropene & Siu & 5 iugh \\
\hline LFW 76 & 81198 & 1, 2-0ibromo-3-chtoropropeno & $5 ! U$ & $5 \log 2$ \\
\hline LFW78 & 81198 & 1,2 -0ibromoethen & siv & $\sin 2$ \\
\hline LFW 78 & 81198 & 1.2-Dichlordobenzens & $5 \mathrm{iv}$ & $5[\mathrm{Lg} h$ \\
\hline LFW 78 & 81196 & 1,2-0ichlorodthane & $5 \mathrm{siv}$ & $5 \log 2$ \\
\hline LFW 78 & 81198 & 1.2. Oichioropropane & 510 & $5 \log 2$ \\
\hline LFT & 81198 & 1.4-Oichiorobenzens & siv & 5 ugh \\
\hline LFW T8 & 91198 & $2+$ thexanom & 1010 & $10 \log$ \\
\hline LFW TG & 81198 & Acetions & 100 & $3.22 \operatorname{lug} 2$ \\
\hline LFW & 81108 & Acetonisitio (Nothy cyenido) & 2010 & $20 \log$ \\
\hline LFW 78 & 61128 & Acrothin & $20 \mid 0$ & $20 \log 2$ \\
\hline LFWTB & 81120 & Aeryionitits & 50 & 5402 \\
\hline LW 78 & 61190 & Aly chiorios & 100 & $\frac{102}{104 x}$ \\
\hline LFW 78 & 81198 & Aluminum, totel recoweraos & 140 & $1970 \mathrm{ugh}$ \\
\hline LFW 70 & 81190 & 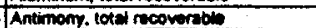 & 270 & 27002 \\
\hline LFT & 81190 & Arsenic totel recovernebs & $\frac{100}{400}$ & 6000 \\
\hline LFW & 81108 & Bapium, loten recowertion & 1.8 & $92,4 \operatorname{ug} 2$ \\
\hline LFW 78 & 81190 & Bonzens & 5|ण & Sluor \\
\hline LWT & 31198 & Bentiom, lotel recovereon & $1.6 \backslash \mathrm{U}$ & $1.6 \operatorname{Lon}$ \\
\hline LFW 78 & 81196 & Bromochloromethem & 5) & 5 ugh \\
\hline LW78 & 81198 & 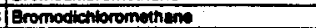 & 50 & $5 \log$ \\
\hline LFW 78 & 81198 & Eromoform & 5ोu & $5 \log 2$ \\
\hline LFW 78 & 81198 & Aromornethense (Metrini bronido) & 1010 & $10 \log x$ \\
\hline LFW76 & 81198 & Cesmitum, lotel rocovernots & $4.7 \mathrm{~J}$ & $1.3 \log$ \\
\hline LFW 78 & $31 \div 98$ & Carbon disulinte & 5ाu & $340 \mathrm{~h}$ \\
\hline LFW 78 & 81128 & Carten ietrachionide & 50 & 5luan \\
\hline LFW78 & 81198 & Chiorobenzens & 5.4 & $5 \lim _{2}$ \\
\hline UFW 78 & 81198 & Chioroethene & $1 0 \longdiv { 4 }$ & $10 \log 2$ \\
\hline LFW 78 & 81196 & Chioroetnenes (vintichionide) & 104 & 101402 \\
\hline LFW 78 & 81196 & Chloroform & Stu & $5 \log 2$ \\
\hline LFW T8 & 81198 & 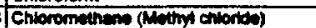 & $101 \mathrm{U}$ & toluoh \\
\hline IFW & 31198 & Chloreprens. & stu & siugh \\
\hline LFW 78 & 81196 & Chromium, totes reconeratos & I! & 28.51002 \\
\hline LFW & 81798 & 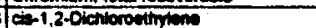 & Stए & 5 \\
\hline LFW 78 & 8119 & 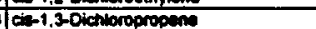 & 50 & $5 / 002$ \\
\hline LFWT & 81198 & 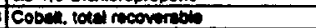 & 4.51 & $.60 \mathrm{un}$ \\
\hline FW & 81100 & I Capour totel rocovernotes & 150 & $8.7 \log$ \\
\hline LFW TO & 81100 & Dioromochioromenenes & stu & siug \\
\hline LFW78 & $511 \%$ & 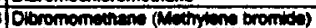 & जाएँ & $3 \log 2$ \\
\hline LFW & 81100 & 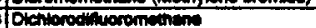 & 100 & 101002 \\
\hline LFW & 81190 & 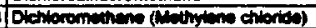 & जए & $2.8410 \mathrm{me}$ \\
\hline LW7 & 81190 & Drowly pithenete & $10.6 \mathrm{~J}$ & 1.241402 \\
\hline LFW 78 & 81498 & Einytoenzense & 50 & $5 \log 2$ \\
\hline LFW78 & 81190 & 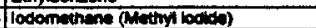 & Siv & STot \\
\hline LFW 76 & 81108 & Iron, total rocoveratos & 74 & 58001002 \\
\hline LF 78 & 81128 & Isocuty weohed & 1000 & $100 \log$ \\
\hline LWT & 81196 & Leed, total reconorabie & $47 \mathrm{j}$ & 4.81002 \\
\hline LFW 78 & 81498 & Metheconionitition & 9010 & 10400 \\
\hline FW 78 & 31198 & Methy einy ketone & 100 & $10 \log$ \\
\hline UFW 78 & 89190 & I Methy inobity kutone & 100 & 101002 \\
\hline LFWT & $81+98$ & Nickel, lotal recovernos & 2015 & $24.2 \log$ \\
\hline LFW 78 & 81198 & ph & $0.1 \mathrm{~J}$ & $5.7 \mathrm{at}$ \\
\hline LFW 78 & 81198 & Propionitith & 5010 & $50 \cos$ \\
\hline LFW 78 & 81198 & Seleniem total nocoverabis & काष & golues \\
\hline LFW 78 & 51198 & Siver, 10 ele necoveretole & जu & 51002 \\
\hline LFW78 & 81198 & Ispectio conductence & 8.9 & 20.81 ustem \\
\hline LFW 78 & 8119 & styrme & 50 & $\sin 2$ \\
\hline LFW 78 & 81196 & Totrichloroetmyions & 5 & $5 \log 2$ \\
\hline LW78 & 1198 & Thallem toty recoverebts & 550 & s5ton \\
\hline LFW 78 & 81198 & Towene & sto & 5 ood \\
\hline LFW 73 & $81+20$ & 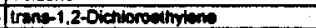 & STu & s.oot \\
\hline LFW 78 & 81198 & trane-1,3-Dichloroprooune & 5 & 5402 \\
\hline LFW 78 & 81198 & trant-1.4-Oichioro-2-bution & 2010 & $20 \cos t$ \\
\hline LWT & 81196 & Trichtoroctingions & 5ीu & $5 \log 2$ \\
\hline Lพ7 & 1119 & 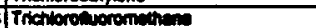 & 5) & $33.8 \cos$ \\
\hline LFW & 81100 & Trtom & 1.44 & $1.17 \mathrm{pclim}$ \\
\hline LFW T8 & 8119 & 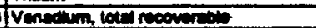 & 8.9 & 34.7 vol \\
\hline एW & B119 & IVnet ectm & 100 & $\frac{10}{10} \operatorname{ung}$ \\
\hline LW 78 & (1190 & $x$ ines & 50 & s.uon \\
\hline LFW 78 & 81190 & 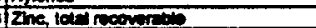 & 53 & $25.4 \operatorname{lon} 2$ \\
\hline
\end{tabular}




\begin{tabular}{|c|}
\hline WEU \\
\hline LFW 20 \\
\hline LFW 20 \\
\hline LW 28 \\
\hline LFW 28 \\
\hline LFW 20 \\
\hline LFW 20 \\
\hline LFW $2 \mathrm{I}$ \\
\hline LFW 20 \\
\hline LFW 28 \\
\hline LFW 28 \\
\hline LFW 28 \\
\hline LFW 20 \\
\hline$\angle F W 28$ \\
\hline LFW 28 \\
\hline LFW 28 \\
\hline LFW 28 \\
\hline LFW 28 \\
\hline LFW 28 \\
\hline LFW 28 \\
\hline LFW 28 \\
\hline LFW 2 \\
\hline LFW 2t \\
\hline LFW 28 \\
\hline FW 28 \\
\hline LFW 28 \\
\hline LFW 2: \\
\hline LFW 20 \\
\hline LFW 28 \\
\hline LFW 20 \\
\hline LFW 28 \\
\hline LW 2 \\
\hline LFW 20 \\
\hline LFW 28 \\
\hline LFW 29 \\
\hline LFW 20 \\
\hline LF 28 \\
\hline LW 20 \\
\hline LFW 20 \\
\hline LF 20 \\
\hline LFW 28 \\
\hline LFW 28 \\
\hline LFW 20 \\
\hline LFW 20 \\
\hline LF" \\
\hline LF 20 \\
\hline LF 28 \\
\hline LFW20 \\
\hline $1+28$ \\
\hline LFW 20 \\
\hline WW 20 \\
\hline LF 20 \\
\hline LFW 28 \\
\hline LFW23 \\
\hline LFW 28 \\
\hline LFW 28 \\
\hline $1+W^{26}$ \\
\hline LF 28 \\
\hline LFW 28 \\
\hline LFW 28 \\
\hline LFW 28 \\
\hline LFW 28 \\
\hline LF 28 \\
\hline LFW 20 \\
\hline LFW2 \\
\hline LFW 28 \\
\hline LFW 28 \\
\hline LF 28 \\
\hline LFW 28 \\
\hline 告W \\
\hline LFW 20 \\
\hline LFW 28 \\
\hline LF 20 \\
\hline LFW 28 \\
\hline IFW 28 \\
\hline LFW 28 \\
\hline 5.W 28 \\
\hline LF 2: \\
\hline LFW \\
\hline $1 F W 30$ \\
\hline 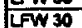 \\
\hline LFW 30 \\
\hline LFW 30 \\
\hline$L F W 30$ \\
\hline LF 30 \\
\hline LFW 30 \\
\hline LFW 30 \\
\hline$L F W 30$ \\
\hline LFW 30 \\
\hline LFW 30 \\
\hline LF 30 \\
\hline FW 30 \\
\hline LW 30 \\
\hline LFW 30 \\
\hline LFW 30 \\
\hline LFW 30 \\
\hline LFW 30 \\
\hline LFW 30 \\
\hline
\end{tabular}




\begin{tabular}{|c|}
\hline LFW 30 \\
\hline LFW 30 \\
\hline LFW 30 \\
\hline LFW 30 \\
\hline LFW 30 \\
\hline LFW 30 \\
\hline LFW 30 \\
\hline LFW 30 \\
\hline LFW 30 \\
\hline LFW 30 \\
\hline LFW 30 \\
\hline LFW 30 \\
\hline LFW 30 \\
\hline$L F W 30$ \\
\hline LFW 30 \\
\hline LFW 30 \\
\hline LFW 30 \\
\hline LFW 30 \\
\hline LFW 30 \\
\hline LFW 30 \\
\hline LFW 30 \\
\hline LFW 30 \\
\hline LFW 30 \\
\hline LFW 30 \\
\hline LFW 30 \\
\hline LFW 30 \\
\hline LFW 30 \\
\hline LFW 30 \\
\hline LFW 36 \\
\hline LFW 30 \\
\hline LFW 30 \\
\hline LFW 30 \\
\hline LFW 30 \\
\hline LFW 30 \\
\hline LFW 30 \\
\hline IN 30 \\
\hline LFW 30 \\
\hline LF 30 \\
\hline LF 30 \\
\hline LFW 30 \\
\hline LW 30 \\
\hline FW 30 \\
\hline LFW 30 \\
\hline LF 30 \\
\hline LFW 30 \\
\hline LFW 30 \\
\hline LFW 30 \\
\hline LF 30 \\
\hline $5 W 30$ \\
\hline UFW 30 \\
\hline LW 30 \\
\hline LFW 30 \\
\hline LFW 30 \\
\hline LFW 30 \\
\hline IFW 30 \\
\hline LF 30 \\
\hline LF 30 \\
\hline LFW 31 \\
\hline LFW 31 \\
\hline LFW 31 \\
\hline IFW 34 \\
\hline LFW 31 \\
\hline LFW 31 \\
\hline LFW 34 \\
\hline LFW 31 \\
\hline LFW 31 \\
\hline LFW 31 \\
\hline LFW 31 \\
\hline LFW 39 \\
\hline LFW 31 \\
\hline LFW 31 \\
\hline LFW 31 \\
\hline LFW31 \\
\hline LFW 31 \\
\hline LFW 31 \\
\hline LFW 39 \\
\hline LFIT \\
\hline LFW 34 \\
\hline LWJ1 \\
\hline LFW \\
\hline LFW 31 \\
\hline LFW 31 \\
\hline LFW 31 \\
\hline LFW 31 \\
\hline LFW \\
\hline LFW 31 \\
\hline LFW 31 \\
\hline LFW 31 \\
\hline LFW 31 \\
\hline LFW 31 \\
\hline LFW 31 \\
\hline LFW 31 \\
\hline LFW 31 \\
\hline FW 31 \\
\hline UFW 31 \\
\hline LFW 31 \\
\hline LFW 31 \\
\hline LFW 31 \\
\hline
\end{tabular}




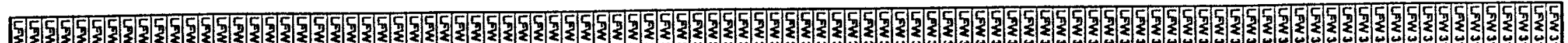

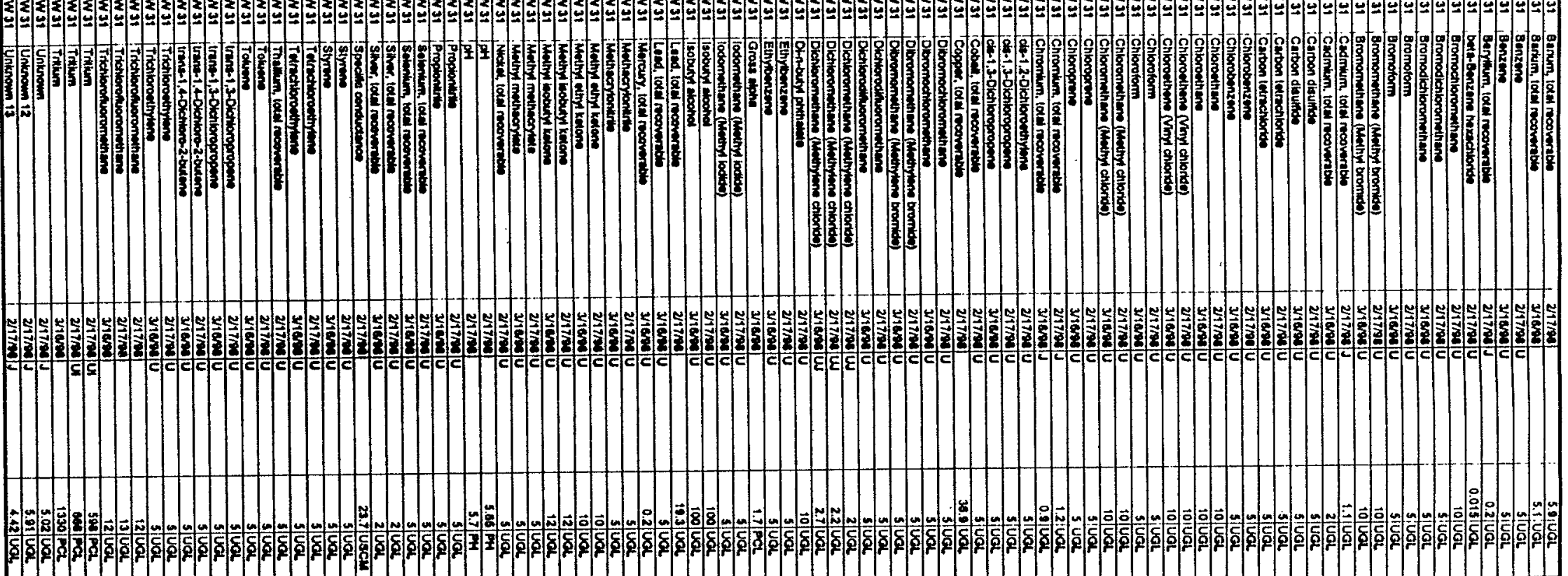




\begin{tabular}{|c|}
\hline LFW31 \\
\hline LFW 34 \\
\hline LFW31 \\
\hline LF 31 \\
\hline LFW 31 \\
\hline LFW 31 \\
\hline FW 31 \\
\hline LFW 32 \\
\hline LFW 32 \\
\hline LFW 32 \\
\hline LFW 32 \\
\hline LFW 32 \\
\hline LFW 32 \\
\hline LFW 32 \\
\hline LFW 32 \\
\hline LFW 32 \\
\hline LF 32 \\
\hline LFW 32 \\
\hline LFW 32 \\
\hline LFW 32 \\
\hline FW 32 \\
\hline IFW 32 \\
\hline LFW 32 \\
\hline LFW 32 \\
\hline LFW 32 \\
\hline LFW 32 \\
\hline FW 32 \\
\hline FW 32 \\
\hline LFW 32 \\
\hline LF 32 \\
\hline FW 32 \\
\hline LFW 32 \\
\hline LFW 32 \\
\hline TW 32 \\
\hline LF 33 \\
\hline LFW 32 \\
\hline LFW 32 \\
\hline LFW 33 \\
\hline LFW 32 \\
\hline LFW 32 \\
\hline LF 32 \\
\hline LFW 32 \\
\hline LFW 32 \\
\hline LFW 32 \\
\hline LFW32 \\
\hline FW 32 \\
\hline LFW32 \\
\hline Wพ 32 \\
\hline FW 32 \\
\hline LF 32 \\
\hline LFW 32 \\
\hline LFW 32 \\
\hline LFW 32 \\
\hline LFW 32 \\
\hline LFW 32 \\
\hline FW 32 \\
\hline LFW 32 \\
\hline LFW 32 \\
\hline LFW 32 \\
\hline FW 32 \\
\hline LFW 32 \\
\hline LFW 32 \\
\hline LFW 32 \\
\hline LFW 32 \\
\hline LFW 32 \\
\hline LFW 32 \\
\hline LFW 32 \\
\hline LFW 32 \\
\hline LFW 32 \\
\hline LFW 32 \\
\hline LFW 32 \\
\hline LF 32 \\
\hline LFW 32 \\
\hline LFW \\
\hline LFW 32 \\
\hline LFW 12 \\
\hline LFW 32 \\
\hline LFW 32 \\
\hline LFY 33 \\
\hline LFW 32 \\
\hline LFW 32 \\
\hline FW 32 \\
\hline LFW 32 \\
\hline LW 32 \\
\hline LFW 32 \\
\hline LFW 32 \\
\hline LW 32C \\
\hline LFW 3XC \\
\hline LFW 32C \\
\hline LFW 320 \\
\hline IFW $32 C$ \\
\hline LFW $32 C$ \\
\hline LFW 32C \\
\hline LFW 326 \\
\hline LFW 326 \\
\hline LFW 32C \\
\hline LFW 32C \\
\hline LFW $32 \mathrm{C}$ \\
\hline
\end{tabular}




\begin{tabular}{|c|}
\hline LW $32 \mathrm{C}$ \\
\hline LFW 32C \\
\hline LFW 32C \\
\hline LFW 320 \\
\hline LFW 32C \\
\hline LFW 32C \\
\hline$L F W 32 C$ \\
\hline LFW 32C \\
\hline LFW 32C \\
\hline LFW 32C \\
\hline LFW 32C \\
\hline LFW 32C \\
\hline LFW $32 C$ \\
\hline LFW 32C \\
\hline LFW $32 \mathrm{C}$ \\
\hline LFW 32C \\
\hline LFW $32 \mathrm{C}$ \\
\hline LFW 32C ! \\
\hline $5 W 320$ \\
\hline$L F W 32 C$ \\
\hline LFW 32C \\
\hline LFW 32C \\
\hline LFW $32 \mathrm{C}$ \\
\hline LFW 32C \\
\hline FW 32C \\
\hline LFW 32C \\
\hline LF $32 C$ \\
\hline LFW 32C \\
\hline LFW $32 C$ \\
\hline LFW 32C \\
\hline LFW $32 C$ \\
\hline LFW $32 \mathrm{C}$ \\
\hline LFW $32 \mathrm{C}$ \\
\hline LFW $32 \mathrm{C}$ \\
\hline FW 32C \\
\hline LFW 32C \\
\hline LFW 32C \\
\hline LFW $32 \mathrm{C}$ \\
\hline LFW 32C \\
\hline LFW 32C \\
\hline LFW 32C \\
\hline FF 322 \\
\hline LFW 32C \\
\hline IFW $32 \mathrm{C}$ \\
\hline LF $32 C$ \\
\hline LFW $32 \mathrm{C}$ \\
\hline LFW $32 \mathrm{C}$ \\
\hline LF 32C \\
\hline LFW $32 \mathrm{C}$ \\
\hline LFW $32 x$ \\
\hline LFW 32C \\
\hline SF 32C \\
\hline LW $32 \mathrm{C}$ \\
\hline LFW $32 \mathrm{C}$ \\
\hline LFW 32C \\
\hline Fw 32c \\
\hline LFW 32C \\
\hline LW $32 \mathrm{C}$ \\
\hline LFW $32 \mathrm{C}$ \\
\hline $1 F W 32 C$ \\
\hline LFW 32C \\
\hline LFW 32C \\
\hline LFW 32C \\
\hline LF 32C \\
\hline IFW 32C \\
\hline LFW 36 \\
\hline LF 34 \\
\hline LFW 34 \\
\hline LF 34 \\
\hline LFW 34 \\
\hline LW 34 \\
\hline IFW 34 \\
\hline LFW 34 \\
\hline LFW 36 \\
\hline LFW 34 \\
\hline FW 38 \\
\hline LW 34 \\
\hline LFW 34 \\
\hline LFW34 \\
\hline IfW 34 \\
\hline JW 34 \\
\hline LFW \\
\hline fF 34 \\
\hline LFW 34 \\
\hline LFW 34 \\
\hline $15 W^{34}$ \\
\hline LFW 36 \\
\hline LFW \\
\hline LFW 34 \\
\hline LFW 34 \\
\hline LFW 34 \\
\hline LFW 34 \\
\hline LFW34 \\
\hline LFW34 \\
\hline LFW 34 \\
\hline LFW 34 \\
\hline FW 34 \\
\hline LFW 34 \\
\hline
\end{tabular}




\begin{tabular}{|c|}
\hline LFW 34 \\
\hline LFW 34 \\
\hline LW 34 \\
\hline LFW \\
\hline LFW 34 \\
\hline FW 3A \\
\hline LPW 34 \\
\hline LFW 34 \\
\hline LFW 34 \\
\hline LFW 34 \\
\hline LFW 34 \\
\hline LFW 34 \\
\hline LFW 34 \\
\hline LFW 34 \\
\hline LF 34 \\
\hline LFW 34 \\
\hline LFW 34 \\
\hline LFW34 \\
\hline LFW 34 \\
\hline LFW34 \\
\hline LFW 34 \\
\hline LFW34 \\
\hline LFW 34 \\
\hline LFWM \\
\hline LFW 34 \\
\hline LFW \\
\hline LFW3 \\
\hline LFW 34 \\
\hline LFW 34 \\
\hline LFW 34 \\
\hline LFWM \\
\hline LFW 34 \\
\hline LFW 34 \\
\hline LFW34 \\
\hline LFW34 \\
\hline LFW34 \\
\hline LFW 34 \\
\hline LFW3 \\
\hline LFW 3 \\
\hline LFW \\
\hline LFW \\
\hline LFW36 \\
\hline LFWM \\
\hline LFW \\
\hline LFW34 \\
\hline LFW 34 \\
\hline LF 3a \\
\hline LFW 439 \\
\hline LFW 130 \\
\hline $5 \% 432$ \\
\hline LFW 438 \\
\hline LFW 438 \\
\hline LFW 439 \\
\hline LFW 438 \\
\hline LFW 439 \\
\hline LFW 438 \\
\hline LFW 438 \\
\hline LFW 438 \\
\hline IFW 43E \\
\hline FW 438 \\
\hline LFW 438 \\
\hline $5 W 438$ \\
\hline LFW 430 \\
\hline $1 F W+30$ \\
\hline LFW 430 \\
\hline LFW 438 \\
\hline LF 138 \\
\hline LFW 430 \\
\hline FW 430 \\
\hline$\angle F W 430$ \\
\hline LFW 430 \\
\hline LFW 430 \\
\hline LFW 439 \\
\hline FW 439 \\
\hline LFW 430 \\
\hline FW 43 \\
\hline LFW 430 \\
\hline LFW 430 \\
\hline FW 43T \\
\hline LFW 430 \\
\hline FW 430 \\
\hline LFW 430 \\
\hline LFW 430 \\
\hline LFW 43a \\
\hline LFW 439 \\
\hline LFW 438 \\
\hline FW 430 \\
\hline $1 \% W 438$ \\
\hline LFW 438 \\
\hline LFW 430 \\
\hline LFW 438 \\
\hline LF 43 \\
\hline LFW 430 \\
\hline LFW 438 \\
\hline LW +38 \\
\hline FW 430 \\
\hline LFW 438 \\
\hline LFW 436 \\
\hline
\end{tabular}




\begin{tabular}{|c|}
\hline$L F W 438$ \\
\hline LFW 438 \\
\hline LFW 430 \\
\hline$L F+38$ \\
\hline LFW 438 \\
\hline$L F W 438$ \\
\hline LFW 438 \\
\hline LFW 438 \\
\hline LFW 438 \\
\hline LFW 43B \\
\hline$L F W 43 B$ \\
\hline LFW 438 \\
\hline FW 438 \\
\hline$L F W 438$ \\
\hline LFW 438 \\
\hline LFW 438 \\
\hline LFW 438 \\
\hline LFW 438 \\
\hline LFW 438 \\
\hline LFW $43 B$ \\
\hline LFW 436 \\
\hline$L F<38$ \\
\hline LFW 638 \\
\hline FW 43B \\
\hline LF 438 \\
\hline LFW 438 \\
\hline LFW 438 \\
\hline LFW 438 \\
\hline FW+38 \\
\hline LFW 438 \\
\hline LFW 43B \\
\hline LFW 438 \\
\hline LFW 438 \\
\hline 15 W 438 \\
\hline UFW 438 \\
\hline LFW 438 \\
\hline LFW 438 \\
\hline LFW 438 \\
\hline LF 438 \\
\hline LFW 438 \\
\hline LFW 438 \\
\hline WW 438 \\
\hline LFW 438 \\
\hline LFW 438 \\
\hline$L F+430$ \\
\hline LFW 439 \\
\hline LFW 430 \\
\hline LFW 430 \\
\hline LFW 438 \\
\hline LW 439 \\
\hline LF 436 \\
\hline LFW 438 \\
\hline LFW 43B \\
\hline FW 438 \\
\hline LFW $\$ 38$ \\
\hline LFW 438 \\
\hline$L F+38$ \\
\hline LW 43B \\
\hline LFW 438 \\
\hline LFW 438 \\
\hline$L F W+38$ \\
\hline LFW 438 \\
\hline LFW 438 \\
\hline LFW 438 \\
\hline LFW 438 \\
\hline LFW 438 \\
\hline LFW 438 \\
\hline LFW 438 \\
\hline LFW 438 \\
\hline LFW 438 \\
\hline $5 F$ 438 \\
\hline FW 436 \\
\hline LFW 43B \\
\hline $5 \times W 430$ \\
\hline LFW 438 \\
\hline$L F W 438$ \\
\hline LFW 430 \\
\hline LFW 438 \\
\hline LFW 430 \\
\hline LFW 438 \\
\hline LF 438 \\
\hline LFW 438 \\
\hline LFW 43B \\
\hline LF 430 \\
\hline LFW 438 \\
\hline LFW 438 \\
\hline LFW 438 \\
\hline FW 438 \\
\hline LFW $430^{\circ}$ \\
\hline LFW 430 \\
\hline$L F W$ 438 \\
\hline IFF 438 \\
\hline LFW 438 \\
\hline LW 438 \\
\hline LFW 43E \\
\hline LFW 438 \\
\hline LFW 438 \\
\hline LFW 438 \\
\hline
\end{tabular}




\begin{tabular}{|c|}
\hline LFW 438 \\
\hline LW 438 \\
\hline LFW 438 \\
\hline LFW 43B \\
\hline LFW 438 \\
\hline LFW 438 \\
\hline LFW 438 \\
\hline LFW 438 \\
\hline LFW 43B \\
\hline LFW 438 \\
\hline LFW 438 \\
\hline LFW 43B \\
\hline$L F W 438$ \\
\hline LFW 438 \\
\hline LFW 438 \\
\hline LFW 430 \\
\hline LFW 435 \\
\hline$L F W 438$ \\
\hline LFW 438 \\
\hline LFW 438 \\
\hline LFW 438 \\
\hline LFW 439 \\
\hline LFW 43B \\
\hline LFW 438 \\
\hline LFW 438 \\
\hline LFW 430 \\
\hline LFW 438 \\
\hline LFW 438 \\
\hline LFW 438 \\
\hline FW 438 \\
\hline LFW 438 \\
\hline LF.38 \\
\hline FW 438 \\
\hline LFW 438 \\
\hline LW 438 \\
\hline LFW 436 \\
\hline LFW 430 \\
\hline$L F W 438$ \\
\hline LFW/SO \\
\hline LFW 438 \\
\hline LFW 438 \\
\hline FW 439 \\
\hline FW 438 \\
\hline 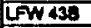 \\
\hline TFW438 \\
\hline $5 F W 436$ \\
\hline LF 438 \\
\hline LFW 436 \\
\hline LFW 438 \\
\hline FW 438 \\
\hline UFW 439 \\
\hline FW 438 \\
\hline LFW 436 \\
\hline LFW 430 \\
\hline$L F W 438$ \\
\hline LFW $43 C$ \\
\hline FW $43 C$ \\
\hline LF+3C \\
\hline FW $43 \mathrm{C}$ \\
\hline LFW $43 C$ \\
\hline LF 43C \\
\hline LFW $43 C$ \\
\hline LW $43 C$ \\
\hline$\overline{F W} 43 \mathrm{C}$ \\
\hline LFW $43 C$ \\
\hline LFW43C \\
\hline LW 43C \\
\hline LFW 43C \\
\hline LFW $43 \mathrm{C}$ \\
\hline LFW 43C \\
\hline FW 43C \\
\hline LFW $43 \mathrm{C}$ \\
\hline FW 43C \\
\hline UFW $43 \mathrm{C}$ \\
\hline LF 43C \\
\hline IFW $4 B$ \\
\hline $5 \bar{F} 43 C$ \\
\hline LFW 43C \\
\hline $5 W+3 C$ \\
\hline FW 43C \\
\hline LFW $43 c$ \\
\hline LF $43 C$ \\
\hline LW $43 C$ \\
\hline LFW $43 C$ \\
\hline SW 43C \\
\hline FW $43 C$ \\
\hline LW $43 C$ \\
\hline LF+3C \\
\hline LFW $43 \mathrm{C}$ \\
\hline LFW $43 \mathrm{C}$ \\
\hline LFW $43 C$ \\
\hline $15 w 43 c$ \\
\hline$L F W 43 C$ \\
\hline LFW $43 \mathrm{C}$ \\
\hline LFW 43C \\
\hline LFW $43 \bar{C}$ \\
\hline LFW 43C \\
\hline LFW $43 \mathrm{C}$ \\
\hline
\end{tabular}




\begin{tabular}{|c|}
\hline LFW 43C \\
\hline LFWAJC \\
\hline LFW 43C \\
\hline LFW 43C \\
\hline LFW 43C \\
\hline$\angle F W 43 C$ \\
\hline $\mathrm{LFW} \triangle 3 \mathrm{C}$ \\
\hline LFW $43 \mathrm{C}$ \\
\hline IFW 43C \\
\hline LFW 43C \\
\hline LFW 43C \\
\hline$L F W 43 C$ \\
\hline LFW A3C \\
\hline LFW $43 \mathrm{C}$ \\
\hline LFW $43 \mathrm{C}$ \\
\hline LFW 43C \\
\hline LFW 43C \\
\hline IFW 43C \\
\hline $\mathrm{LFW} 43 \mathrm{C}$ \\
\hline LFW 43C \\
\hline LFW $43 \mathrm{C}$ \\
\hline LFW 43C \\
\hline LFW \&JC \\
\hline LFW 43C \\
\hline LFW aJC \\
\hline$\angle F W 43 C$ \\
\hline LWW 43C \\
\hline LFW $13 \mathrm{C}$ \\
\hline $5 W 43 C$ \\
\hline$\angle F W 43 C$ \\
\hline$\angle F W 43 C$ \\
\hline$\angle F W 43 C$ \\
\hline FW $43 C$ \\
\hline LFW $13 \mathrm{C}$ \\
\hline$\angle F W 43 C$ \\
\hline$L F W 43 C$ \\
\hline FW $43 C$ \\
\hline LW $13 \mathrm{C}$ \\
\hline LFW $43 C$ \\
\hline$\angle F W 43 C$ \\
\hline LFW $43 \mathrm{C}$ \\
\hline$F W+3 C$ \\
\hline LFW $43 \mathrm{C}$ \\
\hline LW 43C \\
\hline IFW $43 \mathrm{C}$ \\
\hline LFW $43 \mathrm{C}$ \\
\hline LFW $43 \mathrm{C}$ \\
\hline LFW $43 C$ \\
\hline LFV $\triangle 13 C$ \\
\hline LFW $43 \mathrm{C}$ \\
\hline LFW $43 \mathrm{C}$ \\
\hline $15 W 43 C$ \\
\hline LFW 430 \\
\hline LW $43 C$ \\
\hline LFW $43 \mathrm{C}$ \\
\hline LFW $43 C$ \\
\hline$L F+3 C$ \\
\hline$L F W 43 C$ \\
\hline LEW \\
\hline LFW $43 \mathrm{C}$ \\
\hline$L F W 43 C$ \\
\hline LFW 43C \\
\hline$L F W 43 C$ \\
\hline $\bar{F} W 43 c$ \\
\hline INW43C \\
\hline LFW 43C \\
\hline LW $43 C$ \\
\hline $\mathrm{LFW} 43 \mathrm{C}$ \\
\hline LFW43C \\
\hline LFW $\triangle 3 \mathrm{C}$ \\
\hline LFW $43 C$ \\
\hline LW $\triangle 3 C$ \\
\hline LFW $43 \mathrm{C}$ \\
\hline$\angle F W \triangle 3 C$ \\
\hline LFW $43 \mathrm{C}$ \\
\hline LFW $43 \mathrm{C} C$ \\
\hline FFW $43 \mathrm{C}$ \\
\hline LFW 43C \\
\hline LFW $43 C$ \\
\hline LFW $43 \mathrm{C}$ \\
\hline LFW $43 C$ \\
\hline$L F 43 C$ \\
\hline LFW $43 C$ \\
\hline$[F W \triangle 3 C$ \\
\hline$\overline{F W} 43 \mathrm{C}$ \\
\hline $\mathrm{LFW} 43 \mathrm{C}$ \\
\hline LFW $43 \mathrm{C}$ \\
\hline LFW $43 C$ \\
\hline LW $43 C$ \\
\hline LFW $43 C$ \\
\hline LN $43 C$ \\
\hline FW $43 C$ \\
\hline$\angle F W+3 C$ \\
\hline LFW $43 \mathrm{C}$ \\
\hline IFW $43 \mathrm{C}$ \\
\hline LFW $43 \mathrm{C}$ \\
\hline LFW $43 C$ \\
\hline LFWA3C \\
\hline
\end{tabular}




\begin{tabular}{|c|}
\hline LFW $43 C$ \\
\hline LFW 43C \\
\hline LFW $43 C$ \\
\hline LFW $43 C$ \\
\hline LFW $43 \mathrm{C}$ \\
\hline $1 F W 43 C$ \\
\hline LFW 43C \\
\hline LFW $43 C$ \\
\hline$\angle F W+3 C$ \\
\hline$L F W+3 C$ \\
\hline$L F+3 C$ \\
\hline LFW $43 C$ \\
\hline$L F W{ }^{13 C}$ \\
\hline$\angle F W \triangle 13 C$ \\
\hline LFW $43 \mathrm{C}$ \\
\hline$L F W 43 C$ \\
\hline LFW $43 \mathrm{C}$ \\
\hline$L F W+3 C$ \\
\hline LFW $43 \mathrm{C}$ \\
\hline$\angle F W+3 C$ \\
\hline LFW $43 C$ \\
\hline LFW 43C \\
\hline LW $43 \mathrm{C}$ \\
\hline $\mathrm{LFW} 43 \mathrm{C}$ \\
\hline$L F+3 C$ \\
\hline LFW 43C \\
\hline LFW $\mathrm{ASC}$ \\
\hline LFW $43 \mathrm{C}$ \\
\hline LFW 43C \\
\hline LFW $43 C$ \\
\hline LFW $43 C$ \\
\hline LFW $43 \mathrm{C}$ \\
\hline LFW \\
\hline LFW 430 \\
\hline $5 W 43 C$ \\
\hline LFW $+3 C$ \\
\hline LFW 430 \\
\hline$L F W+3 C$ \\
\hline LFW $43 \mathrm{C}$ \\
\hline LFW 43C \\
\hline LFW $43 C$ \\
\hline FFW $43 \mathrm{C}$ \\
\hline LFW $43 \mathrm{C}$ \\
\hline LFW \\
\hline$L F W+3 C$ \\
\hline LFW $43 C$ \\
\hline LNW 43C \\
\hline LF $43 C$ \\
\hline LFW \\
\hline$\angle F W+3 C$ \\
\hline LFW 43 C \\
\hline FW.3C \\
\hline LF $43 C$ \\
\hline LFW $13 C$ \\
\hline LF $13 C$ \\
\hline LFW 43C \\
\hline LFW $43 C$ \\
\hline LFW $43 C$ \\
\hline LFW $43 C$ \\
\hline $5 F+3 C$ \\
\hline WW $43 \mathrm{C}$ \\
\hline LFW $+3 C$ \\
\hline LFW $\triangle 3 C$ \\
\hline$L F W \sqrt{3 C}$ \\
\hline$L F W+3 C$ \\
\hline LFW A3C \\
\hline$L F W+3 C$ \\
\hline LFW $43 \mathrm{C}$ \\
\hline LF $\triangle 13 C$ \\
\hline LFW 430 \\
\hline IFW $43 C$ \\
\hline LFW $43 \mathrm{C}$ \\
\hline LFW $43 \mathrm{C}$ \\
\hline LW 430 \\
\hline $1=W+3 C$ \\
\hline$\angle F W 43 C$ \\
\hline LFW $43 C$ \\
\hline LFWASC \\
\hline$L F W+3 C$ \\
\hline LF 43 \\
\hline LW $43 C$ \\
\hline LW \\
\hline FW $43 C$ \\
\hline LFW $43 \mathrm{C}$ \\
\hline LFW \\
\hline LFW $43 \mathrm{C}$ \\
\hline$\angle F W A 3 C$ \\
\hline LFW $43 C$ \\
\hline LWW 43 \\
\hline LFW $43 C$ \\
\hline LFW $43 C$ \\
\hline LFW $43 \mathrm{C}$ \\
\hline LWW $3 \mathrm{C}$ \\
\hline LFW 43C \\
\hline L्w \\
\hline$L F W+3 C$ \\
\hline$L F W 43 C$ \\
\hline LFW $43 C$ \\
\hline
\end{tabular}




\begin{tabular}{|c|}
\hline LFW 43C \\
\hline LFW 4 3C \\
\hline LFW 4 $3 C$ \\
\hline LFW 43C \\
\hline$L F W 43 C$ \\
\hline LFW $43 C$ \\
\hline$\angle F W 43 C$ \\
\hline LFW $43 \mathrm{C}$ \\
\hline$L F W+3 C$ \\
\hline LFW $43 \mathrm{C}$ \\
\hline LFW A3C \\
\hline LFW 43C \\
\hline LFW $43 \mathrm{C}$ \\
\hline$L F W+3 C$ \\
\hline LFW A3C \\
\hline LFW $43 \mathrm{C}$ \\
\hline LFW 43C \\
\hline $\mathrm{LFW}+3 \mathrm{C}$ \\
\hline LFW $43 C$ \\
\hline$L F+3 C$ \\
\hline LFW $43 \mathrm{C}$ \\
\hline LFW \&3C \\
\hline$L F W 43 C$ \\
\hline FF $43 \mathrm{C}$ \\
\hline IFW $43 \mathrm{C}$ \\
\hline LFW 43C \\
\hline LFW $43 \mathrm{C}$ \\
\hline LFW $43 C$ \\
\hline LFW $43 \mathrm{C}$ \\
\hline LFW $43 C$ \\
\hline LFW $43 C$ \\
\hline LFW $43 C$ \\
\hline$F W 43 C$ \\
\hline$L F W+3 C$ \\
\hline LFW 43C \\
\hline LFW $43 C$ \\
\hline $5 \% 43 C$ \\
\hline LF $43 C$ \\
\hline$L F+3 C$ \\
\hline LFW $43 C$ \\
\hline LFW $43 \bar{C}$ \\
\hline LFW $+3 C$ \\
\hline LPW 43 \\
\hline $5 F<3 C$ \\
\hline $\mathrm{LFW}+3 \mathrm{C}$ \\
\hline$L F W \triangle 3 C$ \\
\hline LFW $43 \mathrm{C}$ \\
\hline LFW $43 C$ \\
\hline$L F W-3 C$ \\
\hline$L F W 43 C$ \\
\hline$L F 43 C$ \\
\hline$L F W=3 C$ \\
\hline$L F W 43 C$ \\
\hline$L F W 43 C$ \\
\hline$L F+3 C$ \\
\hline LFW+3C \\
\hline LFW $\$ 3 C$ \\
\hline$\angle F W+3 C$ \\
\hline FW $43 C$ \\
\hline$L F W 43 C$ \\
\hline LFW 43C \\
\hline LFW $43 C$ \\
\hline LFW 43C \\
\hline$\angle F W+3 C$ \\
\hline IFW $43 C$ \\
\hline LF $43 C$ \\
\hline$L F W+3 C$ \\
\hline LFW $43 C$ \\
\hline$L F W$ \&3C \\
\hline$L F W / 43 C$ \\
\hline$L W{ }^{43 C}$ \\
\hline LFW $43 C$ \\
\hline LFW $43 C$ \\
\hline UNW $43 C$ \\
\hline LFW \\
\hline LFW $43 C$ \\
\hline$L F W \cdot 3 C$ \\
\hline LFW $43 \mathrm{C}$ \\
\hline$L F W$ 4 $3 C$ \\
\hline LFW $43 C$ \\
\hline$F W+3 C$ \\
\hline$L F+3 C$ \\
\hline$\angle F W \angle 3 C$ \\
\hline FW $43 C$ \\
\hline LFW $43 C$ \\
\hline LFW $43 C$ \\
\hline LFW $43 \mathrm{C}$ \\
\hline LFW 43C \\
\hline$\angle F W+3 C$ \\
\hline LFW 43C \\
\hline LFW $43 C$ \\
\hline LFW $43 C$ \\
\hline UF 430 \\
\hline LFW 130 \\
\hline FW 430 \\
\hline$F F$ 4 130 \\
\hline LFW 430 \\
\hline$L F W 430$ \\
\hline
\end{tabular}




\begin{tabular}{|c|}
\hline$L F W 430$ \\
\hline LFW 4 430 \\
\hline$L F W 430$ \\
\hline LFW 430 \\
\hline LFW 430 \\
\hline FF 430 \\
\hline LFW 430 \\
\hline LFW 430 \\
\hline LFW 430 \\
\hline$\angle F W 430$ \\
\hline LFW 430 \\
\hline LFW 430 \\
\hline LFW 430 \\
\hline$L F W \quad 430$ \\
\hline LFW 430 \\
\hline$F_{W} 430$ \\
\hline LFW 430 \\
\hline LF 430 \\
\hline LFW 430 \\
\hline LFW 430 \\
\hline LFW 430 \\
\hline LFW 430 \\
\hline$L F W 430$ \\
\hline LFW 430 \\
\hline LFW 430 \\
\hline$L F \times 430$ \\
\hline LFW 430 \\
\hline$L F+30$ \\
\hline LFW 430 \\
\hline LFW 43D \\
\hline LFW 430 \\
\hline LFW 430 \\
\hline LFW 430 \\
\hline LFW 430 \\
\hline LF 430 \\
\hline LFW 430 \\
\hline$L F 4_{430}$ \\
\hline LFW 430 \\
\hline LF 430 \\
\hline LFW 430 \\
\hline LFW 430 \\
\hline LFW 450 \\
\hline LFW 430 \\
\hline$L F 430$ \\
\hline NW450 \\
\hline LFW 430 \\
\hline LFW 400 \\
\hline$L F W<30$ \\
\hline LFW 430 \\
\hline LFW 430 \\
\hline LF 430 \\
\hline LFW 430 \\
\hline $5 \times 130$ \\
\hline FW 430 \\
\hline $1 F W 430$ \\
\hline WW 430 \\
\hline LFW 430 \\
\hline LW 430 \\
\hline LFW 430 \\
\hline LW 430 \\
\hline LF 430 \\
\hline LFW 430 \\
\hline LFW 430 \\
\hline LFW 430 \\
\hline$L F+30^{\circ}$ \\
\hline UW 430 \\
\hline LFW 430 \\
\hline $5 W 430$ \\
\hline LFW 430 \\
\hline LFW 430 \\
\hline LFW 430 \\
\hline LFW 430 \\
\hline LFW 430 \\
\hline IFW 430 \\
\hline LF 430 \\
\hline LF 430 \\
\hline LFW 43D \\
\hline LF 430 \\
\hline $5=W 430$ \\
\hline$L F W 430$ \\
\hline LFW 430 \\
\hline LFW 430 \\
\hline $15 \times 430$ \\
\hline LFW 430 \\
\hline$L F 430$ \\
\hline LFW 430 \\
\hline LFW 430 \\
\hline IF 430 \\
\hline $5 W 430$ \\
\hline$L F W=30$ \\
\hline 5 F 430 \\
\hline $5 \times 430$ \\
\hline $5 \times 430$ \\
\hline$L W W^{430}$ \\
\hline $4=W 430$ \\
\hline LFW 430 \\
\hline LFW 430 \\
\hline $4 \times 630$ \\
\hline
\end{tabular}




\begin{tabular}{|c|}
\hline LFW 420 \\
\hline LFW 430 \\
\hline LFW 430 \\
\hline FW 430 \\
\hline LFW 430 \\
\hline LFW 430 \\
\hline LFW 430 \\
\hline LFW 430 \\
\hline LFW 430 \\
\hline LFW 430 \\
\hline LFW 430 \\
\hline LFW 430 \\
\hline LFW 43D \\
\hline LFW 430 \\
\hline LFW 430 \\
\hline LFW 430 \\
\hline LFW 430 \\
\hline LFW 430 \\
\hline LFW 430 \\
\hline LW 430 \\
\hline $5 F 430$ \\
\hline LFW 430 \\
\hline LPW 430 \\
\hline LFW 430 \\
\hline LFW 430 \\
\hline LFW 430 \\
\hline LFW 430 \\
\hline LF 430 \\
\hline LFW 430 \\
\hline$F W+30$ \\
\hline LFW430 \\
\hline LFW 430 \\
\hline LFW 430 \\
\hline LFW 430 \\
\hline LW 430 \\
\hline FFW 430 \\
\hline LFW 430 \\
\hline LW 430 \\
\hline LFW 430 \\
\hline LFW 430 \\
\hline LFW 430 \\
\hline LFW 430 \\
\hline LFW 430 \\
\hline LFW 430 \\
\hline LFW 430 \\
\hline$F=430$ \\
\hline$F W 430$ \\
\hline LFW 430 \\
\hline LFW 430 \\
\hline LFW 430 \\
\hline FW 430 \\
\hline LFW 430 \\
\hline LFW 430 \\
\hline FW 430 \\
\hline LFW 430 \\
\hline LFW 430 \\
\hline LFW 43D \\
\hline LFW 430 \\
\hline $5 W 430$ \\
\hline LFW 430 \\
\hline LW +30 \\
\hline $15 W 430$ \\
\hline LW 430 \\
\hline FW 430 \\
\hline LFW 430 \\
\hline LFW 430 \\
\hline LFW 430 \\
\hline LFW 430 \\
\hline 50430 \\
\hline IFW 430 \\
\hline LFW 430 \\
\hline LFW 430 \\
\hline LFW 430 \\
\hline LFW 430 \\
\hline LFW 430 \\
\hline LFW 430 \\
\hline FW 430 \\
\hline$F=W_{430}$ \\
\hline FW 430 \\
\hline LFW 430 \\
\hline LFW 430 \\
\hline LFW 430 \\
\hline LFW 430 \\
\hline FW 430 \\
\hline LFW 430 \\
\hline LW 430 \\
\hline LFW 430 \\
\hline LFW 430 \\
\hline LF 430 \\
\hline LFW 430 \\
\hline LFW 430 \\
\hline LFW 430 \\
\hline$L F W 430$ \\
\hline LFW 430 \\
\hline$F W 430$ \\
\hline FW 430 \\
\hline FW 430 \\
\hline LFW 430 \\
\hline
\end{tabular}




\begin{tabular}{|c|}
\hline LFW 130 \\
\hline LFW 430 \\
\hline LFW 430 \\
\hline LFW 74C \\
\hline LFWTAC \\
\hline$\angle F W 74 C$ \\
\hline LFW 74C \\
\hline LFW 74C \\
\hline LFW 74C \\
\hline LFW 74C \\
\hline LFW 74C \\
\hline Low 74C \\
\hline LFWT4C \\
\hline LWT4C \\
\hline LFW 74C \\
\hline LFW 74C \\
\hline LFW TAC \\
\hline LFW 7AC \\
\hline LFW T\&C \\
\hline LW $74 \mathrm{C}$ \\
\hline LFW 74C \\
\hline LFW TSC \\
\hline LFTCC \\
\hline LFWTC \\
\hline LFWTAC \\
\hline LFW 74C \\
\hline$\angle F W T 4 C$ \\
\hline LFW74C \\
\hline LFW 74C \\
\hline LFW T4C \\
\hline LFWTAC \\
\hline LFW 74C \\
\hline LFW 74C \\
\hline LFWTAC \\
\hline LFW 74C \\
\hline LFTC \\
\hline LFW74C \\
\hline LFW TAC \\
\hline LFWTAC \\
\hline LFW $74 \mathrm{C}$ \\
\hline LFWTAC \\
\hline LFWTAC \\
\hline FWTAC \\
\hline LFWTAC \\
\hline LFW 74C \\
\hline LFW $74 C$ \\
\hline LFW \\
\hline WW 74C \\
\hline LW $74 \mathrm{C}$ \\
\hline LFW T4C \\
\hline LFW 74C \\
\hline LFWTAC \\
\hline FWTAC \\
\hline LFW 74C \\
\hline LFW 74C \\
\hline LFW 74C \\
\hline LFW 74C \\
\hline LFW \\
\hline LFW 74C \\
\hline LF T4C \\
\hline LFW $14 \mathrm{C}$ \\
\hline LFW 74C \\
\hline LFW 74C \\
\hline LFW 7LC \\
\hline LFW $74 C$ \\
\hline FFW T\&C \\
\hline LFW 74C \\
\hline LFW $74 \mathrm{C}$ \\
\hline LWTAC \\
\hline LFWTAC \\
\hline LFWTAC \\
\hline HFW 74C \\
\hline LFWTAC \\
\hline LFWTAC \\
\hline LFW T4C \\
\hline LFWTAC \\
\hline LFWTC \\
\hline LFW74C \\
\hline LFTIC \\
\hline LFWTCC \\
\hline LFW 74 \\
\hline LFWTAC \\
\hline LFW74C \\
\hline LFW74C \\
\hline LFWAC \\
\hline LFW T4C \\
\hline LW74C \\
\hline LFW TAC \\
\hline LFW 7AC \\
\hline LFW 74C \\
\hline LFW 7AC \\
\hline LFW 74C \\
\hline FW $74 \mathrm{C}$ \\
\hline LW74C \\
\hline LWTAC \\
\hline \\
\hline LFW7AC \\
\hline HW 7AC \\
\hline
\end{tabular}




\begin{tabular}{|c|}
\hline LFW 7AC \\
\hline LFW 74C \\
\hline LFW T4C \\
\hline LFW 74C \\
\hline \\
\hline LFW $74 C$ \\
\hline LFW 74C \\
\hline LFW T4C \\
\hline LFW 74C \\
\hline LFW TAC \\
\hline LFW 74C \\
\hline LFW 74C \\
\hline LFW 74C \\
\hline LFW 74C \\
\hline LFW 74C \\
\hline LFW74C \\
\hline LFW 74C \\
\hline LFWTAC \\
\hline LFW $14 C$ \\
\hline LFW 74C \\
\hline LW 74C \\
\hline LFWT4C \\
\hline LFW TIC \\
\hline LFW TAC \\
\hline LFW TAC \\
\hline LFW74C \\
\hline LFW 74C \\
\hline LF 74C \\
\hline LFW74C \\
\hline LFW TAC \\
\hline LFW TAC \\
\hline LFW 74C \\
\hline LFW 7\&C \\
\hline LF $74 C$ \\
\hline LFW TAC \\
\hline LFW 74C \\
\hline LFW T4C \\
\hline LFW74C \\
\hline LFW 74C \\
\hline LFW $74 \mathrm{C}$ \\
\hline LFW 74C \\
\hline LFW TAC \\
\hline LFW 7\&C \\
\hline LFW74C \\
\hline LFW 74C \\
\hline LFW 74C \\
\hline LFW74C \\
\hline LFW 74C \\
\hline LFW \\
\hline LFW74C \\
\hline LFW T4C \\
\hline LFW 74C \\
\hline $5 W 74 C$ \\
\hline LFW 74C \\
\hline LFW 74C \\
\hline LFW TSC \\
\hline LFW 74C \\
\hline LFW 74C \\
\hline LFWTAC \\
\hline SF 74C \\
\hline LFW TAG \\
\hline LFWTAC \\
\hline LFW 74C \\
\hline LFW 746 \\
\hline FW 74C \\
\hline FW74C \\
\hline LFWTAC \\
\hline LFWTAC \\
\hline TFTIC \\
\hline LFWTC \\
\hline LFTAC \\
\hline LFWTAC \\
\hline LFW 74C \\
\hline LW 74C \\
\hline IFWTAC \\
\hline IFW74C \\
\hline UFW 74C \\
\hline FW74C \\
\hline FW 74C \\
\hline $15 \times 746$ \\
\hline LFW.74C \\
\hline FW74C \\
\hline FWT TC \\
\hline FW740 \\
\hline FW740 \\
\hline PW740 \\
\hline FW740 \\
\hline LFW740 \\
\hline FW740 \\
\hline FW 740 \\
\hline FW760 \\
\hline FW 740 \\
\hline LFW740 \\
\hline IFW740 \\
\hline FF 700 \\
\hline$\angle F W 740$ \\
\hline IF 740 \\
\hline LFW 7.0 \\
\hline
\end{tabular}




\begin{tabular}{|c|}
\hline LFW 740 \\
\hline LFW 740 \\
\hline LFW 740 \\
\hline FW 740 \\
\hline LFWT40 \\
\hline LFW T\&D \\
\hline IFW 740 \\
\hline LFW 740 \\
\hline LFW 740 \\
\hline$L \overline{F W} 740$ \\
\hline LFW 740 \\
\hline LFWT40 \\
\hline$L F W 740$ \\
\hline$L F W 740$ \\
\hline LFW 740 \\
\hline$F W 740$ \\
\hline$L F W 760$ \\
\hline$L F W 70$ \\
\hline $15 \times 740$ \\
\hline LFW780 \\
\hline LFW 740 \\
\hline LFW 740 \\
\hline$L F W 740$ \\
\hline LFW740 \\
\hline LFW 740 \\
\hline LFW 740 \\
\hline LFW 740 \\
\hline LPW 740 \\
\hline LFW 740 \\
\hline LFW 740 \\
\hline$L F=740$ \\
\hline LFW 140 \\
\hline LFW 740 \\
\hline LFW740 \\
\hline LFW 740 \\
\hline LFW 740 \\
\hline LFW 740 \\
\hline LFW 760 \\
\hline$\angle F W 740$ \\
\hline LFW 740 \\
\hline $5 \times W 740$ \\
\hline LFW 710 \\
\hline LF/10 \\
\hline LFW 740 \\
\hline LFW740 \\
\hline LFW 740 \\
\hline LFW 740 \\
\hline LFW 740 \\
\hline LPW $\$ 0$ \\
\hline$\angle F W 740$ \\
\hline LFW740 \\
\hline LFW740 \\
\hline LF 740 \\
\hline$\angle F 740$ \\
\hline LFW 740 \\
\hline LFW 740 \\
\hline LFW 740 \\
\hline LFW740 \\
\hline$L F W 740$ \\
\hline Low740 \\
\hline FW 740 \\
\hline$L F W 740$ \\
\hline LFW 75C \\
\hline LFW TSC \\
\hline LFW TBC \\
\hline LFW $75 C$ \\
\hline LFW $75 C$ \\
\hline LFW $73 \mathrm{C}$ \\
\hline LFW TSC \\
\hline LFW $75 C$ \\
\hline LFW TSC \\
\hline LFW 75C \\
\hline FWW 7SC \\
\hline LFW 7SC \\
\hline LFW 75C \\
\hline LFW TSC \\
\hline LFWTSC \\
\hline LFW TSC \\
\hline FW TSC \\
\hline LFW TSE \\
\hline LFW TSC \\
\hline LFWTSC \\
\hline LFW $75 \mathrm{C}$ \\
\hline LFW $75 C$ \\
\hline LFW $73 C$ \\
\hline LFW TSC \\
\hline LFW TSC \\
\hline LFW TSC \\
\hline FW $73 C$ \\
\hline LFW $T S C$ \\
\hline LFW $7 \overline{\text { LC }}$ \\
\hline LFW TSC \\
\hline LFW \\
\hline$L F W 750$ \\
\hline LFW $75 C$ \\
\hline LFW $75 C$ \\
\hline LFW 75C \\
\hline 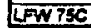 \\
\hline
\end{tabular}




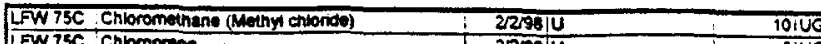

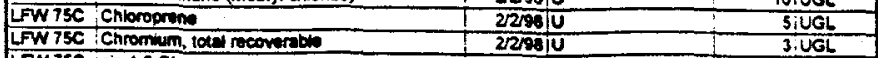

LFW 7SC Eis-1.2-Oichlorowlinime

LFW 75C cis-1,3-Okentorooropent

$2 \sqrt{2}+2$

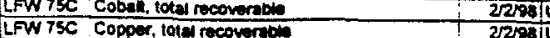

\begin{tabular}{|l|c|c|}
\hline$L F W 75 C$ & 2250 \\
\hline
\end{tabular}

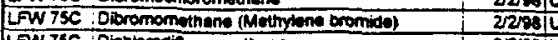

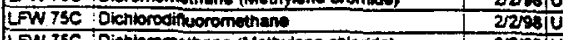

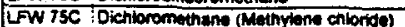

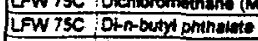

LFW TSC Ethyloenzione

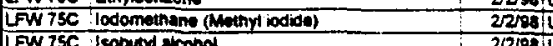

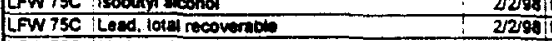

LFW 7SC Methecrionitith

IFW 75C Methy othyl kerone

UFW TSC : Mtotnyt isooutyt ketone

LFW TSC Methri metheartote

LFW 7SC Nicket, todei recowerabe

\begin{tabular}{|l|l|l|l}
\hline LFW $75 \mathrm{C}$ & $\mathrm{pH}$ \\
\hline LFW &
\end{tabular}

LFW 7SC Propionitio

LFWC 21298

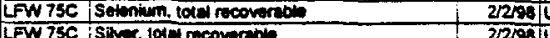

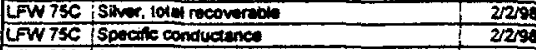

LFW 7SC 5 Styrone

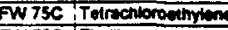

LFW TSC Thallum. lons recovernotio

LFW 75C Tolutine

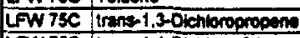

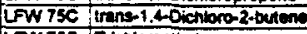

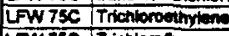

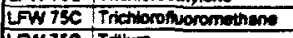

\begin{tabular}{|l|l|l}
\hline LFW 7SC & Trktem \\
\hline LFW
\end{tabular}

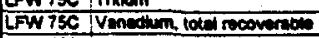

LFW 7SC Vinyl eceiti

LFW 7SC X X yone:

LFW 7SC Zinc, totel recoworete

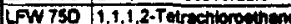

LFW 750 1,1,1. Irichtoroethen

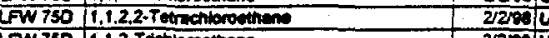

LFW 750 1, 1,2-Thetiloroether

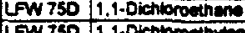

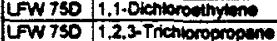

LFW 750 1,2 -Oteromo-3-chloropropenes

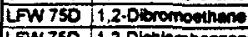

\begin{tabular}{|l|l|l|l}
\hline LFW 750 & $1,2 \cdot$ Dkthlorobenzen \\
\hline
\end{tabular}

LFW 750 1,2-Dichiorocthane

LFW 750 1,2.Okchioropropen.

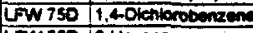

LFW 750 2-Hexumon

LFW750 Acetione

LFW 7SO Acetonitis (Matry oyanide

FW 750, Acrowitis

LFW750, Acryonitin

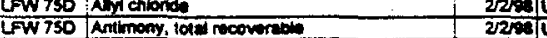

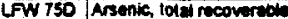

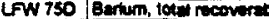

LFW 750 Benzone

(FW

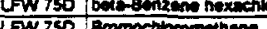

UFW 750 Bromodictiorom the

LFW 750 : Bromotom

UFW 750 Bromomethene (Mothy boomido)

LFW 750 , Cadinim, lorel recoverabe

LFW 750 Carbon disunde

LFW 750 Caroen totrachion

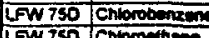

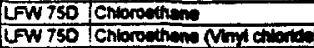

LFW 730 |Chiorolom

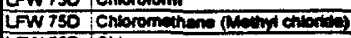

LFW 750 Cnoroore

LFW 750 Chromium, loter recoverese

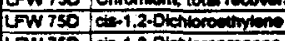

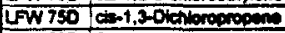

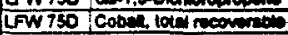

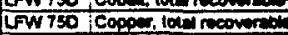

LWW 750 D Doponectiorometren.

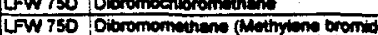

LFW 750 Dibromomithene (Mathy

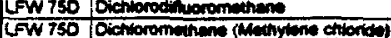

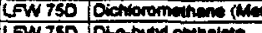

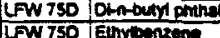

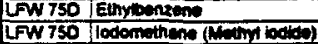

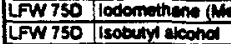

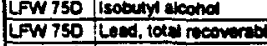

LFW 75D Methecritonit't'

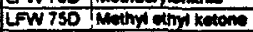

LFW 7SD : Meanyl loothy keton

LFW 750 Netm mothecrice

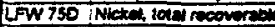

LFW 750 DP

\begin{tabular}{|c|}
\hline IU \\
\hline$\overline{0}$ \\
\hline iv \\
\hline Iu \\
\hline U \\
\hline 0 \\
\hline iv \\
\hline U \\
\hline U \\
\hline iv \\
\hline$\underline{u}$ \\
\hline$u$ \\
\hline $\bar{U}$ \\
\hline $\bar{U}$ \\
\hline U \\
\hline $\bar{U}$ \\
\hline iv \\
\hline $\mathbf{U}$ \\
\hline U \\
\hline 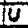 \\
\hline U \\
\hline \\
\hline \\
\hline$U$ \\
\hline$U$ \\
\hline $\bar{U}$ \\
\hline \\
\hline UJ \\
\hline प \\
\hline $\bar{u}$ \\
\hline $\bar{U}$ \\
\hline $\bar{U}$ \\
\hline $\mathbb{U}$ \\
\hline $\bar{U}$ \\
\hline $\mathbf{U}$ \\
\hline \\
\hline$\underline{U}$ \\
\hline $\bar{U}$ \\
\hline $\bar{U}$ \\
\hline \\
\hline U \\
\hline $\bar{u}$ \\
\hline$U$ \\
\hline Ut \\
\hline $\bar{U}$ \\
\hline $\bar{U}$ \\
\hline $\bar{U}$ \\
\hline U \\
\hline$\underline{v}$ \\
\hline $\bar{t}$ \\
\hline $\mathbf{U}$ \\
\hline $\bar{u}$ \\
\hline $\bar{U}$ \\
\hline $\bar{u}$ \\
\hline $\bar{U}$ \\
\hline $\bar{U}$ \\
\hline $\bar{U}$ \\
\hline $\mathbf{v}$ \\
\hline$\overline{\mathbf{U}}$ \\
\hline $\bar{u}$ \\
\hline $\bar{U}$ \\
\hline \\
\hline $\bar{U}$ \\
\hline$\overline{\mathbf{U}}$ \\
\hline $\bar{U}$ \\
\hline $\bar{U}$ \\
\hline $\bar{U}$ \\
\hline$\overline{\mathbf{U}}$ \\
\hline $\bar{U}$ \\
\hline j \\
\hline $\bar{u}$ \\
\hline$\overline{\boldsymbol{U}}$ \\
\hline$\overline{\mathbf{u}}$ \\
\hline $\bar{U}$ \\
\hline$\overline{\mathbf{U}}$ \\
\hline$\overline{\mathbf{U}}$ \\
\hline $\mathbf{u}$ \\
\hline$\overline{\mathbf{U}}$ \\
\hline$\overline{\mathbf{U}}$ \\
\hline $\bar{U}$ \\
\hline $\bar{U}$ \\
\hline$\overline{\mathbf{U}}$ \\
\hline$\overline{\mathbf{U}}$ \\
\hline $\bar{U}$ \\
\hline$\overline{\mathbf{u}}$ \\
\hline $\mathbf{u}$ \\
\hline $\bar{\omega}$ \\
\hline W \\
\hline $\mathbf{U}$ \\
\hline $\mathrm{U}$ \\
\hline $\bar{U}$ \\
\hline \\
\hline$\overline{\mathbf{U}}$ \\
\hline$\overline{0}$ \\
\hline $\bar{U}$ \\
\hline $\mathrm{U}$ \\
\hline $\bar{U}$ \\
\hline$j$ \\
\hline
\end{tabular}




\begin{tabular}{|c|}
\hline LFW TSD \\
\hline $5 \times 750$ \\
\hline LFW 750 \\
\hline $18 W 730$ \\
\hline LFW 750 \\
\hline LFW 750 \\
\hline LFW TSD \\
\hline LFW 750 \\
\hline LFW 750 \\
\hline LFW 750 \\
\hline LFW 75D \\
\hline LFW 7SO \\
\hline LFW 7SD \\
\hline LFW 730 \\
\hline LW 750 \\
\hline LFW 750 \\
\hline$\angle F W 750$ \\
\hline LFW 750 \\
\hline LFW76 \\
\hline LFW 76 \\
\hline LFWT 70 \\
\hline LFWT \\
\hline LFW 76 \\
\hline LW76 \\
\hline LFWT \\
\hline LFW76 \\
\hline LFW 70 \\
\hline LFW 76 \\
\hline LWT6 \\
\hline LW 76 \\
\hline LFW76 \\
\hline LW76 \\
\hline LFW 76 \\
\hline LFT \\
\hline LWT \\
\hline LW76 \\
\hline LWT \\
\hline LFW7 \\
\hline LFW \\
\hline LFWTO \\
\hline LFW 76 \\
\hline LFW 76 \\
\hline LFW76 \\
\hline LW7 \\
\hline LFW76 \\
\hline LFT70 \\
\hline$L F 78$ \\
\hline ENT \\
\hline 1576 \\
\hline LFT \\
\hline FW 76 \\
\hline LFW 76 \\
\hline LF76 \\
\hline FW 76 \\
\hline 1\% 78 \\
\hline LFWT \\
\hline FW 76 \\
\hline LFW7 \\
\hline LFW 76 \\
\hline LFW76 \\
\hline LFW 76 \\
\hline FW78 \\
\hline LFW 76 \\
\hline LFW78 \\
\hline LFW 70 \\
\hline LFW76 \\
\hline LFW70 \\
\hline LW7 \\
\hline LFW76 \\
\hline LFW 76 \\
\hline LF 76 \\
\hline $5 \times 70$ \\
\hline LFW78 \\
\hline LFW7 \\
\hline LFW 78 \\
\hline LFW 70 \\
\hline $15 \times 78$ \\
\hline $15 \times 70$ \\
\hline WW70 \\
\hline LW TO \\
\hline LFW70 \\
\hline FWT \\
\hline FW \\
\hline $5 \times 70$ \\
\hline LF 78 \\
\hline UWT \\
\hline Lพ 78 \\
\hline LFT \\
\hline LFW \\
\hline LFW 76 \\
\hline LFW76 \\
\hline LFW 30 \\
\hline LFW 78 \\
\hline LFW78 \\
\hline LFT \\
\hline 1FW7 \\
\hline LFW 70 \\
\hline LF 70 \\
\hline
\end{tabular}




\begin{tabular}{|c|}
\hline LFW 77 \\
\hline LFWT7 \\
\hline LFWT \\
\hline LFWT \\
\hline LFW? \\
\hline LFWT \\
\hline LFW 77 \\
\hline LFW 77 \\
\hline LFW $\pi$ \\
\hline LFW77 \\
\hline LW7 \\
\hline 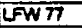 \\
\hline LFW 7 \\
\hline LFW $\pi$ \\
\hline UFW $\pi$ \\
\hline LFW7 \\
\hline LFWT \\
\hline LFW77 \\
\hline LFW \\
\hline LWT \\
\hline LF $\pi$ \\
\hline LF" \\
\hline LFW $\pi$ \\
\hline LFW 7 \\
\hline LFक \\
\hline IFn \\
\hline LFW \\
\hline LFW 77 \\
\hline LFW \\
\hline LFW $\pi$ \\
\hline LFW $\pi$ \\
\hline LFW \\
\hline LF⿳亠口冋 \\
\hline Lwn \\
\hline LFT \\
\hline LW $\pi$ \\
\hline LFW \\
\hline LFW $\bar{n}$ \\
\hline LFW 7 \\
\hline LW7 \\
\hline LFW \\
\hline LFWT \\
\hline LFW $\pi$ \\
\hline LWT \\
\hline LFT \\
\hline LFWT \\
\hline LFW $\bar{T}$ \\
\hline LFT \\
\hline LFT \\
\hline LFWT \\
\hline LFT \\
\hline LFW77 \\
\hline LFW 77 \\
\hline LFWT \\
\hline LFW \\
\hline LFW77 \\
\hline $5 \% \pi$ \\
\hline L्W $\overline{7}$ \\
\hline LFW T7 \\
\hline LFW \\
\hline LFWT \\
\hline LFWT \\
\hline LFW 78 \\
\hline LFW 78 \\
\hline LFW 7 \\
\hline LW 78 \\
\hline LFW 78 \\
\hline LFW78 \\
\hline LWT \\
\hline LFW7 \\
\hline LFW T \\
\hline LFW 76 \\
\hline LFW TE \\
\hline FWTB \\
\hline LFW 78 \\
\hline LFT \\
\hline LFW 78 \\
\hline LF 70 \\
\hline LFW 78 \\
\hline LFW 78 \\
\hline LF 78 \\
\hline LFW 78 \\
\hline LFW \\
\hline LFW TB \\
\hline LFW 7 \\
\hline LFW 78 \\
\hline LFW 70 \\
\hline LFW \\
\hline LFW TA \\
\hline LFW 78 \\
\hline LFW7 \\
\hline LFW 7 , \\
\hline LF 78 \\
\hline LFW \\
\hline LFT \\
\hline LFW \\
\hline LFW 78 \\
\hline LFW \\
\hline
\end{tabular}




\begin{tabular}{|c|c|c|c|}
\hline LFWT & Chioroprone & 2/7/9OU & 5!UGL \\
\hline 나 T T & 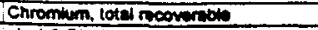 & $217 \% 0$ & $9.110 \mathrm{GL}$ \\
\hline LFW 70 & 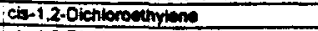 & $2 / 7 / 200$ & S|UGL \\
\hline LFW 78 & Cis-1,3-Otchloropropense & $2517 / 0810$ & SIUGL \\
\hline LFW 78 & Cobal, tola rocoverabis & 2/7/900 & SIUG \\
\hline LFW 78 & Copper. totel rucovernots & 2/17Maj & 1.406 \\
\hline LFW 78 & Dibromochioromethem & $217 \% 00$ & stua \\
\hline LFW TA & Oibromomethane (Methyleme bromide) & $2 / 17 \% 10$ & SUat \\
\hline LFW 70 & Dictionodinuoromethane & $2 / 7 / \mathrm{mog}$ iv & SIVG \\
\hline LFW 78 & Dichloromethene (Methyome chionide) & 2/77rogitu & $2.3: \mathrm{UL}$ \\
\hline LFW 70 & Olnowity phenatele & $2 \mathrm{t}+7 \mathrm{~mol} / \mathrm{J}$ & O.SSIVGL \\
\hline LFW78 & Elhyibenzente & $2 / 479010$ & SIUGL \\
\hline LFW 78 & lodomethen (Mothy iodide) & $217 / 910$ & SIUGL \\
\hline LFW78 & isobuty alcohol & $2 / 179010$ & 1001 Uat \\
\hline FW78 & 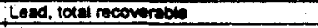 & $2 \sqrt{17 / 981}$ & 0.51 Uat \\
\hline LFW 78 & Methecrytonitile & $2 / 17 / 9010$ & SIUGL \\
\hline LWT: & Mothyt othyt kotone & $2 / 17 / 980$ & $10 \mid \mathrm{UGL}$ \\
\hline LFW78 & Methy bobuin kertone & $2 / 1798 \mathrm{U}$ & 12 Ual \\
\hline LWT78 & Methy meinacryiate & 2179000 & SUG \\
\hline LFW78 & 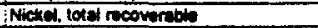 & 21700 & 7.9 uat \\
\hline LFW & H & $2 / 1700$ & $6.46 \mathrm{PH}$ \\
\hline LFW 78 & ph & 21790 & 6.40 PH \\
\hline LFWTE & Propionititio & 2547000 & S/UGL \\
\hline LFW78 & Selenium, tot recoworable & $217 / 00$ & SUat \\
\hline LFW7B & Silver, lotel nocoverabin & 217000 & 2) \\
\hline LFW 78 & Specilfe conductences & $217 \% 0$ & 51.6 USCI \\
\hline LFW 78 & Styrente & 2197000 & 5 UGL \\
\hline LFW78 & Terrectitoroethylions & $2 / 17780$ & Siva \\
\hline LFW 78 & Thallum, total reororate & $217 / 200$ & 5 UGL \\
\hline LFW 78 & Toluons & 217030 & 5 UUGL \\
\hline LFW 78 & trans-1.3-Dichloropropene & $2 / 17 / 900$ & 5IUGL \\
\hline LFW78 & trans-1,4-Oichioro-2-butene & 217190 & 5:UGL \\
\hline LFW78 & Trichloroethylome & $217 / 9010$ & 5.UGL \\
\hline LFW 78 & Trichlorofueromentine & $2 \sqrt{17 / 00}$ & 24 UUG \\
\hline LFW 78 & Trithem & $2017 / 96$ & 1100 PCL \\
\hline LFWTE & Unknowin 15 & 21770915 & 4.76 UGL \\
\hline LFW TS & Unknown i7 & $2 / 17 / 2015$ & 5.081 ua \\
\hline LFWTB & 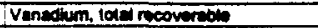 & $2 / 17 / 90$ & 4.5 UUL \\
\hline LFW 79 & Vinyl eavele & $2 / 17000$ & 5.ual \\
\hline LFW79 & Xylenes & 2177rogutu & SUGL \\
\hline LFWTA & 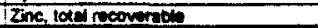 & $2 / 7 / 90$ & 25.814a \\
\hline
\end{tabular}


APPENDIX II FIELD DATA 


\begin{tabular}{|c|}
\hline WEU TO \\
\hline LFW 23 \\
\hline JW 20 \\
\hline UF 2E \\
\hline FW 20 \\
\hline Lw: \\
\hline जि 20 \\
\hline FN 20 \\
\hline LFW 20 \\
\hline LF 30 \\
\hline FW 30 \\
\hline FW30 \\
\hline FW 30 \\
\hline $4 \times 30$ \\
\hline IFW \\
\hline FW 30 \\
\hline LF 30 \\
\hline ज小31 \\
\hline SW31 \\
\hline FW 31 \\
\hline WW31 \\
\hline FW 31 \\
\hline जW31 \\
\hline LFW31 \\
\hline FW31 \\
\hline ज्ञ51 \\
\hline WW \\
\hline WW34 \\
\hline $5 \times 3$ \\
\hline FW3 \\
\hline एwis \\
\hline FW31 \\
\hline LWII \\
\hline$F W 2$ \\
\hline LW 32 \\
\hline F132 \\
\hline FW12 \\
\hline TW2 \\
\hline TWn \\
\hline 1512 \\
\hline IF12 \\
\hline LFW \\
\hline $5 \times 3 x$ \\
\hline FW 320 \\
\hline$F w 3 x$ \\
\hline FW $3 x$ \\
\hline FW 150 \\
\hline$F W 326$ \\
\hline FW $32 \mathrm{C}$ \\
\hline FW 34 \\
\hline $\bar{W}$ \\
\hline $5 \times 34$ \\
\hline FWM \\
\hline जW \\
\hline FW 34 \\
\hline $\operatorname{cow} x$ \\
\hline $5 \times 36$ \\
\hline FW 450 \\
\hline $5 \mathrm{~W} 430$ \\
\hline$\overline{L W} 458$ \\
\hline$F W+30$ \\
\hline $5 \mathrm{FW} 438$ \\
\hline $5 W 438$ \\
\hline FW 430 \\
\hline$\overline{F W} / 30$ \\
\hline $5 \times 430$ \\
\hline $5 \times 430$ \\
\hline FW 436 \\
\hline $5 W 430$ \\
\hline IFW 430 \\
\hline $5 \times 430$ \\
\hline $5 \times 438$ \\
\hline LFW 45 \\
\hline FW 450 \\
\hline FW 43 \\
\hline FW 43 \\
\hline $5 W+39$ \\
\hline $2 \times 139$ \\
\hline FW 436 \\
\hline LW 439 \\
\hline $5 \times 436$ \\
\hline FW 436 \\
\hline $\mathrm{JW} 43 \mathrm{C}$ \\
\hline IFWASC \\
\hline LFise \\
\hline IF $4 S C$ \\
\hline I $43 C$ \\
\hline $5 \times 43 C$ \\
\hline IFW 43C \\
\hline FW $43 C$ \\
\hline LFW 43C \\
\hline FW $43 C$ \\
\hline LFW $43 \mathrm{C}$ \\
\hline LF 630 \\
\hline IFW 43C \\
\hline LFW \\
\hline$\sqrt{F+3 C}$ \\
\hline LFWasc \\
\hline
\end{tabular}

\begin{tabular}{|c|c|c|}
\hline LF⿳3口 & Jwertele & We्य \\
\hline जि $4 \bar{X}$ & Were Tenpoins & 2था \\
\hline FW & Wher Tomperture & ytose \\
\hline FW $43 C$ & Werer Tamperetere & बase \\
\hline IFW & ph & $2 \sqrt{2}$ \\
\hline LW+3C & pH & עreal \\
\hline LFW $43 C$ & ph & Gewe \\
\hline TW 430 & Ar Penporatere & 2000 \\
\hline FW 430 & Air Tempermens & $3 / 100$ \\
\hline FW 430 & Air Tomperature & ares \\
\hline FW 430 & 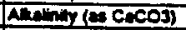 & $2 \sqrt{200}$ \\
\hline $5 \% 4+30$ & An & Miand \\
\hline$\sqrt{\mathrm{F}} 4 \mathbf{3 0}$ & Anener (es caco3) & eant \\
\hline TW +30 & 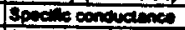 & 2र्य \\
\hline LFW 430 & Specte cendudereses & Inew \\
\hline FW 130 & 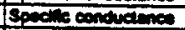 & $\cos$ \\
\hline IF 430 & Truotide & $2 \sqrt{200}$ \\
\hline FW 50 & Trution & S160 \\
\hline FW 430 & Tuter & $\sin$ \\
\hline $5 \% 430$ & Volmin pored & $22 \pi$ \\
\hline G\% 430 & Volume Purpes & 3/60 \\
\hline LFW 430 & Volperis & $\sin$ \\
\hline $5 \times 430$ & womlen & $2 \sqrt{201}$ \\
\hline LFW430 & Whenen & प्रres: \\
\hline FW 430 & wertow & Weat \\
\hline FWas0 & Were Ten & $2 \sqrt{2}=$ \\
\hline FW 4SO & 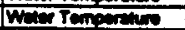 & अ1क्त \\
\hline FW & 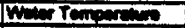 & $\sin$ \\
\hline SW 430 & H & 2क्य! \\
\hline FW & 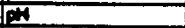 & अ1की \\
\hline FW430 & P & ons \\
\hline SFTic & Ar Tongentests & 2200 \\
\hline FWTAC & Antind (O Cecos) & 220 \\
\hline FWT4C & 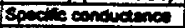 & $2 \sqrt{2 \times 1}$ \\
\hline IFWTAC & Tuovoly & $2 \sqrt{200}$ \\
\hline Wwis & Vowe pored & $2 \pi \approx$ \\
\hline FWTAC & Werten & $2 \sqrt{2 \pi}$ \\
\hline FW74C & 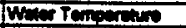 & 2200 \\
\hline IFTAC & DH & $22 \omega$ \\
\hline LFT\$O & A Tenpenters & $2 \pi x$ \\
\hline WW760 & hen (c)cos & 2000 \\
\hline SW760 & Specin oentutentes & $2 \sqrt{2 \pi}$ \\
\hline 5W740 & Troter & $2 \sqrt{20}$ \\
\hline Pि70 & 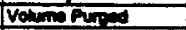 & $2 \Omega 20$ \\
\hline $5=740$ & When & ह2त \\
\hline FW760 & Wor Tonowise & 2क्या \\
\hline SW70 & A & $2 \pi m$ \\
\hline FW7SC & 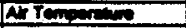 & $22 \omega$ \\
\hline SWTEC & 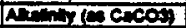 & 2200 \\
\hline FW $75 \mathrm{C}$ & 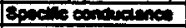 & हथा \\
\hline Firse & Tutidy & 2था \\
\hline FWTSC & Voleme fored & 2था \\
\hline LFWTSC & werlew & $2 \sqrt{200}$ \\
\hline LWTSE & Weter Ten & $2 \sqrt{25}$ \\
\hline FW 7SC & $p+4$ & 22/21 \\
\hline Fiso & Ar Terpentur & 22201 \\
\hline FW750 & Nunty (a) CAcos & $22=1$ \\
\hline FW750 & Spoctis conductenes & $2 \sqrt{289}$ \\
\hline SW750 & Tutioly & 2200 \\
\hline FW 750 & voline ponen & 2250 \\
\hline FW750 & When Len & $2 \sqrt{250}$ \\
\hline FW 750 & Wret Tomperous & $2 \sqrt{201}$ \\
\hline FWT30 & H. & 22201 \\
\hline WW & Ar Temponsers & $217 \%$ \\
\hline 157 & Ar Fomparcues & $2 / 170$ \\
\hline FWT & Almingeson & $21 \%$ \\
\hline twn & 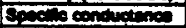 & 21170 \\
\hline जWT & Tut & $217 \%$ \\
\hline FWT & Volum fures & $21 \%$ \\
\hline WW & Volden Pures & 21170 \\
\hline FW & Whinten & 21170 \\
\hline Frn & worlew & 217na \\
\hline WWT & Wert Ton lem & $2 / 7 \%$ \\
\hline FWT & of & $2 / 17 / 2$ \\
\hline WWT & Artenpartios & 250 \\
\hline Lon & Vohmen pores & 200 \\
\hline 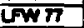 & Wret Low & $20 \%$ \\
\hline WW & Ar Tenpereno & 200 \\
\hline FW7 & 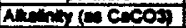 & 2010 \\
\hline 57 & 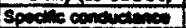 & 20 \\
\hline FWT & Tutos & $2 \pi n$ \\
\hline w7 & Vollons Pugh & 200 \\
\hline WW & Wher Low & $20+1$ \\
\hline जw & 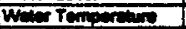 & एक्य \\
\hline एwn & ph & 200 \\
\hline 5W7 & Ar Temporim & 277n \\
\hline FW & NrTenpertus & $217 \%$ \\
\hline LWT & 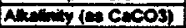 & यानका \\
\hline FWT & 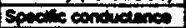 & 217N \\
\hline FWT & Totity & $217 \pi$ \\
\hline SWT & Volume Puper & 21750 \\
\hline IFWTE & Votume pured & $24 \pi$ \\
\hline UWT & Whar Lew & $217 \pi$ \\
\hline WW7 & Werer Low & $2 \sin$ \\
\hline WW & Whe Tenpowing & $217 \pi$ \\
\hline IFW & N & $2 / 17 \%$ \\
\hline
\end{tabular}




\begin{tabular}{|c|c|c|c|c|c|c|c|c|c|c|c|c|c|}
\hline Wall Name & Sampled & Duplo & Time & Cond. & H2O Tmp & $\rho H$ & Turt. & Flow & PAIK & $A / n$ & Alr Tmp & H2O Opdn & Purged \\
\hline LFW 28 & 89198 & & 1338 & 40 & $\begin{array}{r}20.5 \\
\end{array}$ & 5.7 & 4.7 & 1.42 & 8 & 0 & \begin{tabular}{|r}
36.2 \\
\end{tabular} & \begin{tabular}{r|}
26.8 \\
\end{tabular} & 37 \\
\hline LFW 30 & 92698 & & 1054 & 24 & 20.2 & 4.7 & 1.1 & 2 & of & 0 & 20.5 & 42.51 & 22 \\
\hline LFW 30 & 89198 & & 1500 & 25 & 20.3 & 4.8 & 2.5 & 2.6 & 0 & 0 & 38.8 & 42.7 & 39 \\
\hline LFW 3t & 91698 & & 1017 & 31 & 19.6 & 4.8 & 1.2 & 1.36 & 0 & 0 & 24.1 & & 19 \\
\hline LFW 32 & 81298 & & 1055 & 29 & 20.7 & 4.7 & 1.4 & 1.6 & 0 & 0 & 35 & 59.15 & 40 \\
\hline LFW 32C & 92698 & & 1136 & 28 & 19.7 & 4.9 & 1.1 & 0.28 & 0 & 0 & 27.1 & 59.89 & 27 \\
\hline LFW 32C & 81398 & & 803 & 30 & 20.5 & 5.1 & 3.1 & 0.26 & 1 & 0 & 22.7 & 60.2 & 18 \\
\hline LFW 34 & 81198 & & 1416 & 35 & 20.8 & 4.6 & 4.1 & 1.33 & 0 & 0 & 35.8 & 39.03 & 28 \\
\hline LFW 43B & 81098 & & 1253 & 19 & 19.9 & 5.2 & 0.5 & 3.24 & 0 & 0 & 30.1 & 35.78 & 107 \\
\hline LFW $43 C$ & 81098 & & 1338 & 15 & 20.2 & 5.1 & 0.6 & 2.04 & 0 & 0 & 30.6 & 35.15 & 53 \\
\hline LFW 430 & 81098 & & 1216 & 16 & 19.3 & 4.8 & 0.8 & 1 & 0 & 0) & 28.9 & 34.85 & 36 \\
\hline LFW 74C & 81098 & & 1016 & 34 & 20.2 & 4.6 & 2.3 & 2.2 & 0 & 0 & 28 & 49.35 & 91 \\
\hline LFW 740 & 81098 & & 1056 & 54 & 21.3 & 5 & 13.3 & 0.89 & 0) & 0) & 28.9 & 49.5 & 23 \\
\hline LFW $75 C$ & 81298 & & 814 & 32 & 20.4 & 5.2 & 1.8 & 1.79 & 0 & 아 & 25.3 & 33.68 & 43 \\
\hline LFW 750 & 81298 & & 1002 & 45 & 21.1 & 5.6 & 2.6 & 1.41 & 10 & 0 & 31.8 & 33.68 & 24 \\
\hline LFW 78 & 92698 & & 1000 & & & & & 0.28 & &. & 18.2 & 60.51 & 13 \\
\hline LFW 78 & 92698 & $x$ & 1015 & 18 & 21 & 4.7 & 10.4 & 0.26 & 2 & 0 & 19.1 & 60.51 & 1 \\
\hline LFW 76 & 81198 & & 1056 & 29 & 22.1 & 5.4 & 14.9 & 0.28 & 5 & ㅇ. & 29.5 & 80.65 & 13 \\
\hline LFW 77 & 81098 & & 1408 & & & & & $\circ$ & . & & 31 & 56.85 & 4 \\
\hline LFWT & 81098 & $x$ & 1432 & 33 & 21.2 & 5.2 & 150 & 0.28 & 1 & 0 & 31.4 & 56.86 & 2 \\
\hline LFW 78 & 81198 & & 1205 & & + &.$\quad 2$ & & 0.26 & & $E$ & 31 & 75.65 & 8 \\
\hline LFW 78 & 81198 & $x$ & 1235 & 38 & 20.6 & 5.8 & 48.2 & 0.28 & 6) & 0 & 31.2 & 75.65 & 1 \\
\hline
\end{tabular}

Water Elevation Calculations

\begin{tabular}{|c|c|c|c|c|c|}
\hline & Column A & Column B & $\mathrm{A}-\mathrm{B}$ & Column C & $A-C$ \\
\hline & Standpipe elev. & 1Q H2O depth & 1Q H2O elev. & 3QH2O depth & $3 \mathrm{Q} H 2 \mathrm{O}$ elev \\
\hline LFW 28 & 192.6 & 27.9 & \begin{tabular}{r|}
164.7 \\
\end{tabular} & 26.8 & 165.8 \\
\hline LFW 30 & 210.2 & 44.13 & 166.07 & 42.51 & 167.69 \\
\hline LFW 31 & 229.6 & 63.45 & 166.15 & & 229.6 \\
\hline LFW 32 & 223.9 & 60.7 & 163.2 & 59.15 & 164.75 \\
\hline LFW 32C & 222.3 & 61.45 & 160.85 & 59.9 & 162.4 \\
\hline LFW 34 & 201.2 & 39.76 & 161.44 & 39 & 162.2 \\
\hline LFW 43B & 203.2 & 37.06 & 166.14 & 35.78 & 167.42 \\
\hline LFW 43C & 202.8 & 36.7 & 166.1 & 35.15 & 167.65 \\
\hline LFW 43D & 203.1 & 36.25 & 166.85 & 34.85 & 168.25 \\
\hline LFW 74C & 213.8 & 50.6 & 163.2 & 49.35 & 164.45 \\
\hline LFW 74D & 214.1 & 50.85 & 163.25 & 49.5 & 164.6 \\
\hline LFW 75C & 198 & 34.78 & 163.22 & 33.68 & 164.32 \\
\hline LFW 75D & 198.3 & 34.75 & 163.55 & 33.68 & 164.62 \\
\hline LFW 76 & 222 & 61.5 & 160.5 & 60.51 & 161.49 \\
\hline LFW 77 & 222.7 & 58.67 & 164.03 & 56.85 & 165.85 \\
\hline LFW 78 & 239.1 & 76.8 & 162.3 & 75.65 & 163.45 \\
\hline
\end{tabular}




\section{APPENDIX III}

QUALIFIERS 
(Blank)

E

1

$J$

L

M

R

$T$

$u$

V

$Y$

1

2

3

Data are not qualified. Numbers should be interpreted exactly as reported.

The detected result is between the sample-specific EQL and the method detection limit.

The value in the result field is the instrument reading, not the sample quantitation limit. Always used with the resuk qualifier $U$.

Value is estimated because quantitation in the sample or in associated quality control samples did not meet specifications.

Value is off-scale high. The actual value is not known but is known to be greater than the value shown.

Presence of the analyte is veritied but-not quantified.

Result was rejected because performance requirements in the sample analysis or associated quality control analyses were not met.

Analyte was not detected; if present, it was below the criteria for detection.

Material analyzed for but not detected. Analytical result reported is less than the sample quantitation limit.

Analyte was detected in an associated method blank.

Result was obtained from an unpreserved or improperty preserved sample.

Data may not be accurate.

Result may be an underestimation of the irue value due to analytical bias.

Result may be an overestimation of the true value due to analytical bias.

The associated result may be of poor precision (high variability) due to analytical bias.

Note: These are onty some of the qualifiers present in the database. All modifiers associated with the data are published in the result tables of EPD/EMS' quarterty groundwater monitoring reports, the official repository o the data. 